\title{
The TOMCAT global chemical transport model v1.6: description of chemical mechanism and model evaluation
}

\author{
Sarah A. Monks ${ }^{1,2,3}$, Stephen R. Arnold ${ }^{1}$, Michael J. Hollaway ${ }^{1}$, Richard J. Pope ${ }^{1,4}$, Chris Wilson ${ }^{1,4}$, Wuhu Feng ${ }^{1,5}$, \\ Kathryn M. Emmerson ${ }^{6}$, Brian J. Kerridge ${ }^{7}$, Barry L. Latter ${ }^{7}$, Georgina M. Miles ${ }^{7}$, Richard Siddans ${ }^{7}$, and \\ Martyn P. Chipperfield ${ }^{1}$ \\ ${ }^{1}$ Institute for Climate and Atmospheric Science, University of Leeds, Leeds, UK \\ ${ }^{2}$ Chemical Sciences Division, Earth System Research Laboratory, National Oceanic and Atmospheric Administration, \\ Boulder, CO, USA \\ ${ }^{3}$ Cooperative Institute for Research in Environmental Sciences, University of Colorado, Boulder, CO, USA \\ ${ }^{4}$ National Centre for Earth Observation, University of Leeds, Leeds, UK \\ ${ }^{5}$ National Centre for Atmospheric Science, University of Leeds, Leeds, UK \\ ${ }^{6}$ CSIRO Oceans and Atmosphere Flagship, Aspendale, Australia \\ ${ }^{7}$ Remote Sensing Group, STFC Rutherford Appleton Laboratory, Harwell Oxford, UK
}

Correspondence to: Sarah A. Monks (sarah.monks@noaa.gov)

Received: 7 August 2016 - Discussion started: 12 August 2016

Revised: 6 March 2017 - Accepted: 31 May 2017 - Published: 17 August 2017

\begin{abstract}
This paper documents the tropospheric chemical mechanism scheme used in the TOMCAT 3-D chemical transport model. The current scheme includes a more detailed representation of hydrocarbon chemistry than previously included in the model, with the inclusion of the emission and oxidation of ethene, propene, butane, toluene and monoterpenes. The model is evaluated against a range of surface, balloon, aircraft and satellite measurements. The model is generally able to capture the main spatial and seasonal features of high and low concentrations of carbon monoxide (CO), ozone $\left(\mathrm{O}_{3}\right)$, volatile organic compounds (VOCs) and reactive nitrogen. However, model biases are found in some species, some of which are common to chemistry models and some that are specific to TOMCAT and warrant further investigation. The most notable of these biases are (1) a negative bias in Northern Hemisphere $(\mathrm{NH})$ winter and spring $\mathrm{CO}$ and a positive bias in Southern Hemisphere ( $\mathrm{SH}) \mathrm{CO}$ throughout the year, (2) a positive bias in $\mathrm{NH} \mathrm{O}_{3}$ in summer and a negative bias at high latitudes during $\mathrm{SH}$ winter and (3) a negative bias in $\mathrm{NH}$ winter $\mathrm{C}_{2}$ and $\mathrm{C}_{3}$ alkanes and alkenes. TOMCAT global mean tropospheric hydroxyl radical $(\mathrm{OH})$ concentrations are higher than estimates inferred from observations of methyl chloroform but similar to, or lower than, multi-model mean concentrations reported in recent model intercompari-
\end{abstract}

son studies. TOMCAT shows peak $\mathrm{OH}$ concentrations in the tropical lower troposphere, unlike other models which show peak concentrations in the tropical upper troposphere. This is likely to affect the lifetime and transport of important trace gases and warrants further investigation.

\section{Introduction}

Atmospheric chemistry plays a central role in air quality and climate change, which can have a negative effect on humans on a global scale. Air pollution has been estimated to have caused over 3 million deaths worldwide in 2010, and this rate is estimated to double by 2050 due to projected increases in emissions (Lelieveld et al., 2015). Increases in anthropogenic emissions have led to higher atmospheric concentrations of greenhouse gases, such as methane $\left(\mathrm{CH}_{4}\right)$ and ozone $\left(\mathrm{O}_{3}\right)$, contributing significantly to the observed rise in global mean surface temperature (Stocker et al., 2013). Chemical processing, emissions and transport determine the concentrations and distribution of pollutants within the atmosphere and the impact that they have on society. Reactive gases, such as volatile organic compounds (VOCs) and nitrogen oxides $\left(\mathrm{NO}_{x}\right)$, influence air quality and climate as they result in the 
formation of $\mathrm{O}_{3}$ and aerosols. Other gases such as carbon monoxide (CO), which may not directly affect the climate, can have secondary impacts by influencing the lifetime of gases such as $\mathrm{CH}_{4}$ (Berntsen et al., 2005).

Atmospheric chemistry models help to inform our understanding of how atmospheric chemistry affects climate and air quality on a global or regional scale. These models can be used to simulate the temporal and spatial evolution of important short-lived pollutants, taking into account the main physical and chemical processes that act on trace constituents in the troposphere (emissions, chemistry, transport and deposition). The chemical and dynamical complexity and the spatial resolution of such models is a compromise between model accuracy and computational efficiency. Atmospheric chemistry models are often run as chemical transport models (CTMs), where transport is driven by reanalysis products that assimilate meteorological observations. This allows the simulated chemical fields to provide context for measurements, which are often limited spatially and temporally. They can also be used to further understand the impacts of new atmospheric processes that have been identified by measurements (e.g. Lelieveld et al., 2008). CTMs are of particular use in investigating the impacts of natural and anthropogenic emissions on atmospheric burdens of pollutants that are important for air quality and climate reasons and for sourcereceptor studies for policy-making purposes (e.g. Sanderson et al., 2008; Fiore et al., 2009).

The TOMCAT CTM is a three-dimensional (3-D) global Eulerian model that has been used for a wide range of tropospheric and stratospheric chemistry studies. For example, it has been used to investigate the impacts of $\mathrm{O}_{3}$ on crop yields (Hollaway et al., 2012), fire emissions on Arctic interannual variability (Monks et al., 2012) and to identify the main sources of peak summertime $\mathrm{O}_{3}$ in the Mediterranean (Richards et al., 2013). In the stratosphere, the model has been used to study issues such as ozone depletion (e.g. Chipperfield et al., 2015) and the impact of solar variability (e.g. Dhomse et al., 2013). TOMCAT is also the host model for the GLOMAP aerosol module (Mann et al., 2010).

This paper summarises the current tropospheric chemical mechanism scheme used in TOMCAT (Sect. 2). The scheme gives a more detailed representation of hydrocarbon chemistry than previously included in the model, with the inclusion of the emission and oxidation of ethene, propene, butane, toluene and monoterpenes. Alkenes have the greatest potential for forming $\mathrm{O}_{3}$ (Saunders et al., 2003), and previously, isoprene was the only alkene treated in the TOMCAT model. In addition, a more extensive VOC scheme makes it possible to couple the TOMCAT tropospheric chemistry to the formation of secondary organic aerosol in future versions of the GLOMAP aerosol model (Mann et al., 2010). Key gas-phase species simulated by the latest version of the model are shown and evaluated using a range of observations. The model simulations that are evaluated are described in Sect. 2.2 and the observations that are used are described

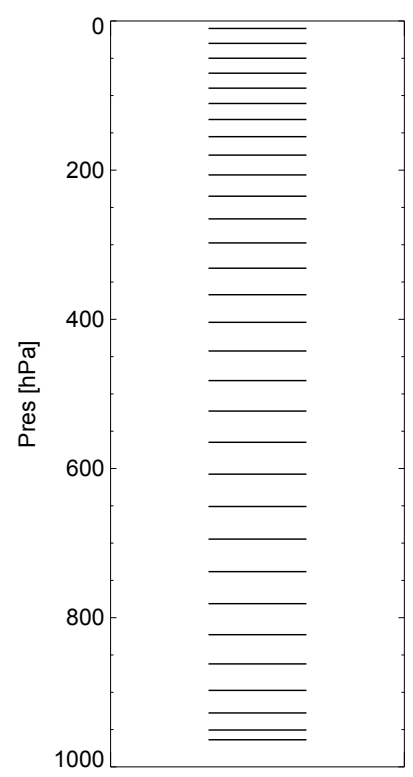

Figure 1. Global annual mean pressure levels from the TOMCAT model.

in Sect. 3. The observational platforms that are used include surface, satellite, aircraft and balloon sounding measurements. The model results and comparisons with observations are shown in Sect. 4 and focus on annual, seasonal and monthly mean simulated concentrations. The chemical species that are discussed include $\mathrm{CO}, \mathrm{O}_{3}$, VOCs, reactive nitrogen $\left(\mathrm{NO}_{y}\right)$ and the hydroxyl radical $(\mathrm{OH})$.

\section{The TOMCAT model}

The TOMCAT model is an Eulerian offline 3-D global CTM and is described by Chipperfield (2006). The model has a flexible horizontal and vertical resolution, and the vertical domain can be varied depending on the problem being studied. Typical horizontal resolutions range from $5.6^{\circ} \times 5.6^{\circ}$ for multidecadal stratospheric studies to $1.2^{\circ} \times 1.2^{\circ}$ for short case studies. The model uses a $\sigma-p$ coordinate system, with near-surface levels following the terrain $(\sigma)$ and higher levels $(\sim>100 \mathrm{hPa})$ using pressure levels $(p)$. The model extends from the surface to $\sim 10 \mathrm{hPa}$ for tropospheric simulations, as used in this study. The global mean pressure levels are shown in Fig. 1. Model meteorology is forced by winds, temperature and humidity fields from the European Centre for Medium-Range Weather Forecasts (ECMWF) reanalyses (Dee et al., 2011). These data are read in every $6 \mathrm{~h}$ and linearly interpolated in time to the model time step and to the TOMCAT grid. To avoid inconsistencies between horizontal and vertical winds after this interpolation, the vertical motion is diagnosed from horizontal divergence instead of using analysed vertical velocities. Large-scale tracer advection in the meridional, zonal and vertical directions is based on 
Table 1. TOMCAT annual global emissions used in the model simulations presented in this paper $\left(\operatorname{Tg}(\operatorname{species})\right.$ year $\left.{ }^{-1}\right)$.

\begin{tabular}{lrrrrrr}
\hline Species & Anthropogenic & Fires & Biogenic & Ocean & Soil & Total \\
\hline CO & 595.27 & 331.62 & 76.57 & 20.01 & 1023.47 \\
Ethene & 6.81 & 2.84 & 16.70 & 1.40 & 27.75 \\
Ethane & 6.34 & 1.67 & 0.14 & 0.98 & 9.14 \\
Propene & 3.04 & 1.57 & 6.10 & 1.52 & 12.23 \\
Propane & 5.68 & 0.38 & 0.02 & 1.30 & 7.37 \\
Toluene & 25.34 & 10.66 & 0.26 & & & 36.26 \\
Butane & 12.38 & 0.60 & & & & 12.98 \\
Formaldehyde & 2.99 & 4.13 & 4.03 & & & 11.15 \\
Acetone & 0.54 & 1.86 & 28.58 & & & 30.98 \\
Acetaldehyde & 2.00 & 4.55 & 11.20 & & & 17.75 \\
Methanol & 0.93 & 5.38 & 159.87 & & & 166.18 \\
Isoprene & & 0.80 & 525.84 & & & 526.64 \\
Monoterpenes & & 0.28 & 97.10 & & & 97.37 \\
NO & 107.73 & 19.41 & & & 16.31 & 143.46 \\
\hline
\end{tabular}

the Prather (1986) scheme, which conserves mass and maintains tracer gradients (Chipperfield, 2006). Sub-grid-scale transport (boundary layer mixing and convective transport) is treated in the model using the Holtslag and Bolville (1993) and Tiedtke (1989) schemes. There is also an option to run the model using archived convective mass fluxes (Feng et al., 2011). Wilson et al. (2014) used sulfur hexafluoride $\left(\mathrm{SF}_{6}\right)$ to evaluate model tracer transport and showed that the model is able to reproduce seasonal transport timescales and patterns along with the location of the intertropical convergence zone. However, they also noted that the model interhemispheric transport is somewhat slow, resulting in an interhemispheric gradient in $\mathrm{SF}_{6}$ that was $18 \%$ too large.

Natural and anthropogenic surface emissions are read into the model on a $1^{\circ} \times 1^{\circ}$ resolution and regridded online to the model grid. The model is usually provided with monthly mean emissions, and a temporal interpolation is performed online to the model time step. Isoprene emissions are emitted and then have a diurnal cycle imposed online to account for the dependence of emissions on daylight. Lightning emissions of $\mathrm{NO}_{x}$ are coupled to convection in the model and therefore vary in space and time according to the seasonality and spatial pattern of convective activity (Stockwell et al., 1999).

Dry deposition velocities are weighted by prescribed fixed land cover fields and seasonally varying sea-ice fields from the NCAR community land model (CLM) (Oleson et al., 2010). The 16 CLM land types were regridded onto the model resolution and reclassified into the TOMCAT's five land types (forest, grass/shrub/crop, bare ground, sea ice and water). Chemical species' deposition velocities were then determined based upon time of day and season, and were weighted by the proportion of the grid box covered by each land type. Wet deposition is parameterised according to the proportionality of the removal rate to the concentration of the species and is dependent on convection rates, precip- itation and the solubility of gases. The scheme has been shown to perform well within the TOMCAT model with a $4 \%$ bias compared to radon observations (Giannakopoulos et al., 1999).

\subsection{Tropospheric chemistry scheme}

The previously documented TOMCAT tropospheric chemical mechanism included odd oxygen $\left(\mathrm{O}_{x}\right)$, reactive nitrogen $\left(\mathrm{NO}_{y}\right)$, carbon monoxide, methane, ethane, propane, acetaldehyde, acetone and formaldehyde chemistry (Arnold et al., 2005). TOMCAT also includes oxidation of isoprene based on the Mainz Isoprene Mechanism (MIM) scheme (Pöschl et al., 2000). The implementation of this scheme into TOMCAT is described by Young (2007). Isoprene is one of the largest single sources of VOCs to the atmosphere, accounting for around a third of total natural and anthropogenic VOC emissions (Guenther et al., 2006). After emission, isoprene is highly reactive and can influence $\mathrm{O}_{3}$ concentrations both regionally (Chameides et al., 1988) and globally (Wang and Shallcross, 2000). $\mathrm{O}_{3}, \mathrm{OH}, \mathrm{CO}$ and PAN from the condensed MIM scheme were found to agree within $10 \%$ of the concentrations calculated from a more explicit representation of isoprene chemistry in the Master Chemical Mechanism (Pöschl et al., 2000). However, significant uncertainties still exist in the representation of isoprene chemistry in models, and chemical mechanisms will likely evolve in the future (e.g. Archibald et al., 2010, 2011; Squire et al., 2015). Most recently, the TOMCAT model chemistry has been expanded to include the emission and destruction of some $\mathrm{C}_{2}-\mathrm{C}_{7}$ unsaturated and aromatic hydrocarbons (ethene, propene, toluene and butane) based on the Extended Tropospheric Chemistry scheme (ExtTC), and monoterpenes based on the MOZART3 chemical mechanism (Kinnison et al., 2007). The extended chemistry scheme results in an increase in the global burden of $\mathrm{CO}, \mathrm{O}_{3}, \mathrm{PAN}$ and $\mathrm{HO}_{2}$, and a small decrease in $\mathrm{OH}$ in 
Table 2. Chemical species treated in the tropospheric chemistry scheme of the TOMCAT CTM. If the species are emitted, the "dry deposited" or "wet deposited" categories are marked with a Y in the relevant column. The family column indicates which short-lived species are grouped together for advection and chemistry. TOMCAT abbreviations: Me indicates $\mathrm{CH}_{3}$, Et indicates $\mathrm{C}_{2} \mathrm{H}_{5}, \mathrm{Pr}_{\text {indicates }} \mathrm{C}_{3} \mathrm{H}_{7}, \mathrm{MACR}$ indicates lumped species (methacrolein, methyl vinyl ketone and other C4 carbonyls), HACET indicates hydroxyacetone, MGLY indicates methylglyoxal, NALD indicates nitrooxy acetaldehyde, TERP indicates generic terpene compound, AROM indicates generic aromatic compound, MEK indicates methyl ethyl ketone, Prpe indicates $\mathrm{C}_{3} \mathrm{H}_{7} \mathrm{O}$, ONIT indicates organic nitrate, and $\mathrm{S}$ indicates stratospheric tracer (TOMCAT species 39-43).

\begin{tabular}{|c|c|c|c|c|c|}
\hline & TOMCAT species & Family & Dry deposited? & Wet deposited? & Emitted \\
\hline 1 & $\mathrm{O}\left({ }^{3} \mathrm{P}\right)$ & $\mathrm{O}_{x}$ & & & \\
\hline 2 & $\mathrm{O}\left({ }^{1} \mathrm{D}\right)$ & $\mathrm{O}_{x}$ & & & \\
\hline 3 & $\mathrm{O}_{3}$ & $\mathrm{O}_{x}$ & Y & & \\
\hline 4 & NO & $\mathrm{NO}_{x}$ & Y & & \\
\hline 5 & $\mathrm{NO}_{3}$ & $\mathrm{NO}_{x}$ & $\mathrm{Y}$ & $\mathrm{Y}$ & \\
\hline 6 & $\mathrm{NO}_{2}$ & $\mathrm{NO}_{x}$ & $\mathrm{Y}$ & & $\mathrm{Y}$ \\
\hline 7 & $\mathrm{~N}_{2} \mathrm{O}_{5}$ & & $\mathrm{Y}$ & $\mathrm{Y}$ & \\
\hline 8 & $\mathrm{HO}_{2} \mathrm{NO}_{2}$ & & Y & $\mathrm{Y}$ & \\
\hline 9 & $\mathrm{HONO}_{2}$ & & $\mathrm{Y}$ & $\mathrm{Y}$ & \\
\hline 10 & $\mathrm{OH}$ & & & & \\
\hline 11 & $\mathrm{HO}_{2}$ & & & $\mathrm{Y}$ & \\
\hline 12 & $\mathrm{H}_{2} \mathrm{O}_{2}$ & & $\mathrm{Y}$ & $\mathrm{Y}$ & \\
\hline 13 & $\mathrm{CH}_{4}$ & & & & $\mathrm{Y}$ \\
\hline 14 & $\mathrm{CO}$ & & $\mathrm{Y}$ & & $\mathrm{Y}$ \\
\hline 15 & $\mathrm{HCHO}$ & & $\mathrm{Y}$ & $\mathrm{Y}$ & $\mathrm{Y}$ \\
\hline 16 & $\mathrm{MeOO}$ & & & $\mathrm{Y}$ & \\
\hline 17 & $\mathrm{H}_{2} \mathrm{O}$ & & & & \\
\hline 18 & $\mathrm{MeOOH}$ & & $\mathrm{Y}$ & $\mathrm{Y}$ & \\
\hline 19 & HONO & & $\mathrm{Y}$ & $\mathrm{Y}$ & \\
\hline 20 & $\mathrm{C}_{2} \mathrm{H}_{6}$ & & & & $\mathrm{Y}$ \\
\hline 21 & EtOO & & & & \\
\hline 22 & EtOOH & & $\mathrm{Y}$ & $\mathrm{Y}$ & \\
\hline 23 & $\mathrm{MeCHO}$ & & Y & & $\mathrm{Y}$ \\
\hline 24 & $\mathrm{MeCO}_{3}$ & & & & \\
\hline 25 & PAN & & $\mathrm{Y}$ & & \\
\hline 26 & $\mathrm{C}_{3} \mathrm{H}_{8}$ & & & & $\mathrm{Y}$ \\
\hline 27 & n-PrOO & & & & \\
\hline 28 & i-PrOO & & & & \\
\hline 29 & $\mathrm{n}$-PrOOH & & $\mathrm{Y}$ & $\mathrm{Y}$ & \\
\hline 30 & i-PrOOH & & $\mathrm{Y}$ & $\mathrm{Y}$ & \\
\hline 31 & $\mathrm{EtCHO}$ & & $\mathrm{Y}$ & & \\
\hline 32 & $\mathrm{EtCO}_{3}$ & & & & \\
\hline 33 & $\mathrm{Me}_{2} \mathrm{CO}$ & & $\mathrm{Y}$ & & $\mathrm{Y}$ \\
\hline 34 & $\mathrm{MeCOCH}_{2} \mathrm{OO}$ & & & & \\
\hline 35 & $\mathrm{MeCOCH}_{2} \mathrm{OOH}$ & & $\mathrm{Y}$ & $\mathrm{Y}$ & \\
\hline 36 & PPAN & & $\mathrm{Y}$ & & \\
\hline 37 & $\mathrm{MeONO}_{2}$ & & & & \\
\hline 38 & $\mathrm{O}\left({ }^{3} \mathrm{P}\right) \mathrm{S}$ & $\mathrm{S}_{x}$ & & & \\
\hline 39 & $\mathrm{O}\left({ }^{1} \mathrm{D}\right) \mathrm{S}$ & $\mathrm{S}_{x}$ & & & \\
\hline 40 & $\mathrm{O}_{3} \mathrm{~S}$ & $\mathrm{~S}_{x}$ & $\mathrm{Y}$ & & \\
\hline 41 & NOXS & & $\mathrm{Y}$ & & \\
\hline 42 & $\mathrm{HNO}_{3} \mathrm{~S}$ & & $\mathrm{Y}$ & $\mathrm{Y}$ & \\
\hline 43 & NOYS & & $\mathrm{Y}$ & $\mathrm{Y}$ & \\
\hline 44 & $\mathrm{C}_{5} \mathrm{H}_{8}$ & & & & $\mathrm{Y}$ \\
\hline 45 & $\mathrm{C}_{10} \mathrm{H}_{16}$ & & & & $\mathrm{Y}$ \\
\hline 46 & TERPOOH & & $\mathrm{Y}$ & $\mathrm{Y}$ & \\
\hline 47 & $\mathrm{ISO}_{2}$ & & & & \\
\hline 48 & ISOOH & & $\mathrm{Y}$ & $\mathrm{Y}$ & \\
\hline 49 & ISON & & $\mathrm{Y}$ & $\mathrm{Y}$ & \\
\hline 50 & MACR & & $\mathrm{Y}$ & & \\
\hline
\end{tabular}


Table 2. Continued.

\begin{tabular}{|c|c|c|c|c|c|}
\hline & TOMCAT species & Family & Dry deposited? & Wet deposited? & Emitted \\
\hline 51 & $\mathrm{MACRO}_{2}$ & & & & \\
\hline 52 & MACROOH & & $\mathrm{Y}$ & $\mathrm{Y}$ & \\
\hline 53 & MPAN & & $\mathrm{Y}$ & & \\
\hline 54 & HACET & & $\mathrm{Y}$ & $\mathrm{Y}$ & \\
\hline 55 & MGLY & & $\mathrm{Y}$ & $\mathrm{Y}$ & \\
\hline 56 & NALD & & $\mathrm{Y}$ & & \\
\hline 57 & $\mathrm{HCOOH}$ & & $\mathrm{Y}$ & $\mathrm{Y}$ & \\
\hline 58 & $\mathrm{MeCO}_{3} \mathrm{H}$ & & $\mathrm{Y}$ & $\mathrm{Y}$ & \\
\hline 59 & $\mathrm{MeCO}_{2} \mathrm{H}$ & & $\mathrm{Y}$ & $\mathrm{Y}$ & \\
\hline 60 & $\mathrm{MeOH}$ & & $\mathrm{Y}$ & $\mathrm{Y}$ & $\mathrm{Y}$ \\
\hline 61 & TERPO $_{2}$ & & & & \\
\hline 62 & $\mathrm{C}_{2} \mathrm{H}_{4}$ & & & & $\mathrm{Y}$ \\
\hline 63 & $\mathrm{C}_{2} \mathrm{H}_{2}$ & & & & Y \\
\hline 64 & $\mathrm{C}_{4} \mathrm{H}_{10}$ & & & & $\mathrm{Y}$ \\
\hline 65 & $\mathrm{C}_{3} \mathrm{H}_{6}$ & & & & $\mathrm{Y}$ \\
\hline 66 & AROM & & & & $\mathrm{Y}$ \\
\hline 67 & MEK & & & & \\
\hline 68 & MeCOCOMe & & $\mathrm{Y}$ & $\mathrm{Y}$ & \\
\hline 69 & $\mathrm{BtOO}$ & & & & \\
\hline 70 & PrpeOO & & & & \\
\hline 71 & $\mathrm{AROMO}_{2}$ & & & & \\
\hline 72 & MEKOO & & & & \\
\hline 73 & $\mathrm{BtOOH}$ & & Y & $\mathrm{Y}$ & \\
\hline 74 & PrpeOOH & & $\mathrm{Y}$ & $\mathrm{Y}$ & \\
\hline 75 & AROMOOH & & $\mathrm{Y}$ & $\mathrm{Y}$ & \\
\hline 76 & MEKOOH & & $\mathrm{Y}$ & $\mathrm{Y}$ & \\
\hline 77 & ONIT & & & & \\
\hline 78 & $\mathrm{EtCO}_{3} \mathrm{H}$ & & & & \\
\hline 79 & $\mathrm{EtCO}_{2} \mathrm{H}$ & & & & \\
\hline
\end{tabular}

summer (see the Supplement). Whilst this scheme has been used in the TOMCAT model for some scientific studies (e.g. Richards et al., 2013; Emmons et al., 2015), the expanded scheme was not fully documented. This is the purpose of this study.

The current model chemistry scheme has a total of 79 species, 16 of which are emitted (see Table 2), and approximately 200 chemical reactions. The bimolecular, termolecular and photolysis reactions are shown in Tables 3, 4 and 5, respectively. The chemical reactions are integrated in the model with a 15 min chemical time step using a software package, ASAD (Carver et al., 1997), which allows the use of input files that contain the information listed in Tables 2-5. The package allows fractional products and the use of families, which can be used for grouping very short-lived species together for transportation (see Table 2 for species treated as families). The bimolecular and termolecular kinetic rates are mostly taken from the 2005 International Union of Pure and Applied Chemistry recommendations (http://iupac.pole-ether.fr/) and the Leeds Master Chemical Mechanism (MCM, 2004). Simplified ethene, propene and butane chemistry is based on von Kuhlmann
(2001), with reaction rates taken from IUPAC (Atkinson et al., 2006b). Ethane chemistry adds one bimolecular reaction (R135-R136, Table 3) and one termolecular reaction (R17-19, Table 5) to TOMCAT. Oxidation of ethane by $\mathrm{OH}$ forms PrpeOO, a peroxy radical, which continues in the propene oxidation chain (von Kuhlmann, 2001). Propane oxidation adds 6 bimolecular reactions (R137-145, Table 3) and 1 termolecular reaction to TOMCAT (R17-19, Table 4) and butane adds 10 bimolecular reactions (R123134, Table 3) and 5 photolysis reactions (R40a-46b, Table 5). Ethene, propene and butane emissions are emitted into the respective compounds, with no lumping of higher alkenes/alkanes. Toluene is emitted into a generic aromatic compound, AROM, which produces AROMO2 (peroxy radicals) and AROMOOH (hydroperoxides). Including AROM adds 11 bimolecular reactions to the model (R146-R156, Table 3 ) and 2 photolysis reactions (R48a-R48b, Table 5), with reactions rates taken from Folberth et al. (2006). Including ethene, propene, butane and toluene will account for missing sources of carbon in the model and produces peroxy radicals that are important for $\mathrm{O}_{3}$ production (von Kuhlmann, 2001). Alkenes are particularly efficient at producing $\mathrm{O}_{3}$, with pho- 
Table 3. TOMCAT gas-phase bimolecular reactions. $T$ is the model grid-box temperature in kelvin. Reaction rate references 1: Atkinson et al. (2004a), 2: Atkinson et al. (2005), 3: Atkinson et al. (2006a), 4: MCM (2004), 5: Tyndall et al. (2001), 6: Ravishankara et al. (2002), 7: Pöschl et al. (2000), 8: Kinnison et al. (2007), 9: Folberth et al. (2006).

\begin{tabular}{|c|c|c|c|c|}
\hline & Reactants & Products & $k$ & Reference \\
\hline 1 & $\mathrm{HO}_{2}+\mathrm{NO}$ & $\rightarrow \mathrm{OH}+\mathrm{NO}_{2}$ & $3.60 \times 10^{-12} \exp \left(\frac{270}{T}\right)$ & 2 \\
\hline 2 & $\mathrm{HO}_{2}+\mathrm{NO}_{3}$ & $\rightarrow \mathrm{OH}+\mathrm{NO}_{2}$ & $4.00 \times 10^{-12}$ & 2 \\
\hline 3 & $\mathrm{HO}_{2}+\mathrm{O}_{3}$ & $\rightarrow \mathrm{OH}+\mathrm{O}_{2}$ & $2.03 \times 10^{-16}\left(\frac{T}{300}\right)^{4.57} \exp \left(\frac{693}{T}\right)$ & 2 \\
\hline 4 & $\mathrm{HO}_{2}+\mathrm{HO}_{2}$ & $\rightarrow \mathrm{H}_{2} \mathrm{O}_{2}$ & $2.20 \times 10^{-13} \exp \left(\frac{600}{T}\right)$ & 2 \\
\hline 5 & $\mathrm{HO}_{2}+\mathrm{MeOO}$ & $\rightarrow \mathrm{MeOOH}$ & $3.80 \times 10^{-13} \exp \left(\frac{780}{T}\right)$ & 2 \\
\hline 6 & $\mathrm{HO}_{2}+\mathrm{MeOO}$ & $\rightarrow \mathrm{HCHO}$ & $3.80 \times 10^{-13} \exp \left(\frac{780}{T}\right)$ & 2 \\
\hline 7 & $\mathrm{HO}_{2}+\mathrm{EtOO}$ & $\rightarrow$ EtOOH & $3.80 \times 10^{-13} \exp \left(\frac{900}{T}\right)$ & 2 \\
\hline 8 & $\mathrm{HO}_{2}+\mathrm{MeCO}_{3}$ & $\rightarrow \mathrm{MeCO}_{3} \mathrm{H}$ & $2.08 \times 10^{-13} \exp \left(\frac{980}{T}\right)$ & 2 \\
\hline 9 & $\mathrm{HO}_{2}+\mathrm{MeCO}_{3}$ & $\rightarrow \mathrm{MeCO}_{2} \mathrm{H}+\mathrm{O}_{3}$ & $1.04 \times 10^{-13} \exp \left(\frac{980}{T}\right)$ & 2 \\
\hline 10 & $\mathrm{HO}_{2}+\mathrm{MeCO}_{3}$ & $\rightarrow \mathrm{OH}+\mathrm{MeOO}$ & $2.08 \times 10^{-13} \exp \left(\frac{980}{T}\right)$ & 2 \\
\hline 11 & $\mathrm{HO}_{2}+\mathrm{n}-\mathrm{PrOO}$ & $\rightarrow \mathrm{n}-\mathrm{PrOOH}$ & $1.51 \times 10^{-13} \exp \left(\frac{1300}{T}\right)$ & 4 \\
\hline 12 & $\mathrm{HO}_{2}+\mathrm{i}-\mathrm{PrOO}$ & $\rightarrow \mathrm{i}-\mathrm{PrOOH}$ & $1.51 \times 10^{-13} \exp \left(\frac{1300}{T}\right)$ & 4 \\
\hline 13 & $\mathrm{HO}_{2}+\mathrm{EtCO}_{3}$ & $\rightarrow \mathrm{O}_{2}+\mathrm{EtCO}_{3} \mathrm{H}$ & $3.05 \times 10^{-13} \exp \left(\frac{1040}{T}\right)$ & 4 \\
\hline 14 & $\mathrm{HO}_{2}+\mathrm{EtCO}_{3}$ & $\rightarrow \mathrm{O}_{3}+\mathrm{EtCO}_{2} \mathrm{H}$ & $1.25 \times 10^{-13} \exp \left(\frac{1040}{T}\right)$ & 4 \\
\hline 15 & $\mathrm{HO}_{2}+\mathrm{MeCOCH}_{2} \mathrm{OO}$ & $\rightarrow \mathrm{MeCOCH}_{2} \mathrm{OOH}$ & $1.36 \times 10^{-13} \exp \left(\frac{1250}{T}\right)$ & 4 \\
\hline 16 & $\mathrm{MeOO}+\mathrm{NO}$ & $\rightarrow \mathrm{HO}_{2}+\mathrm{HCHO}+\mathrm{NO}_{2}$ & $2.95 \times 10^{-12} \exp \left(\frac{285}{T}\right)$ & 2 \\
\hline 17 & $\mathrm{MeOO}+\mathrm{NO}$ & $\rightarrow \mathrm{MeONO}_{2}$ & $2.95 \times 10^{-15} \exp \left(\frac{285}{T}\right)$ & 2 \\
\hline 18 & $\mathrm{MeOO}+\mathrm{NO}_{3}$ & $\rightarrow \mathrm{HO}_{2}+\mathrm{HCHO}+\mathrm{NO}_{2}$ & $1.30 \times 10^{-12}$ & 2 \\
\hline 19 & $\mathrm{MeOO}+\mathrm{MeOO}$ & $\rightarrow \mathrm{MeOH}+\mathrm{HCHO}$ & $1.03 \times 10^{-13} \exp \left(\frac{365}{T}\right)$ & 4 \\
\hline 20 & $\mathrm{MeOO}+\mathrm{MeOO}$ & $\rightarrow \mathrm{HO}_{2}+\mathrm{HO}_{2}+\mathrm{HCHO}+\mathrm{HCHO}$ & $1.03 \times 10^{-13} \exp \left(\frac{365}{T}\right)$ & 2 \\
\hline 21 & $\mathrm{MeOO}+\mathrm{MeCO}_{3}$ & $\rightarrow \mathrm{HO}_{2}+\mathrm{HCHO}+\mathrm{MeOO}$ & $1.80 \times 10^{-12} \exp \left(\frac{500}{T}\right)$ & 2 \\
\hline 22 & $\mathrm{MeOO}+\mathrm{MeCO}_{3}$ & $\rightarrow \mathrm{MeCO}_{2} \mathrm{H}+\mathrm{HCHO}$ & $2.00 \times 10^{-13} \exp \left(\frac{500}{T}\right)$ & 2 \\
\hline 23 & $\mathrm{EtOO}+\mathrm{NO}$ & $\rightarrow \mathrm{MeCHO}+\mathrm{HO}_{2}+\mathrm{NO}_{2}$ & $2.60 \times 10^{-12} \exp \left(\frac{380}{T}\right)$ & 2 \\
\hline 24 & $\mathrm{EtOO}+\mathrm{NO}_{3}$ & $\rightarrow \mathrm{MeCHO}+\mathrm{HO}_{2}+\mathrm{NO}_{2}$ & $2.30 \times 10^{-12}$ & 2 \\
\hline 25 & $\mathrm{EtOO}+\mathrm{MeCO}_{3}$ & $\rightarrow \mathrm{MeCHO}+\mathrm{HO}_{2}+\mathrm{MeOO}$ & $4.40 \times 10^{-13} \exp \left(\frac{1070}{T}\right)$ & 2 \\
\hline 26 & $\mathrm{MeCO}_{3}+\mathrm{NO}$ & $\rightarrow \mathrm{MeOO}+\mathrm{CO}_{2}+\mathrm{NO}_{2}$ & $7.50 \times 10^{-12} \exp \left(\frac{290}{T}\right)$ & 2 \\
\hline 27 & $\mathrm{MeCO}_{3}+\mathrm{NO}_{3}$ & $\rightarrow \mathrm{MeOO}+\mathrm{CO}_{2}+\mathrm{NO}_{2}$ & $4.00 \times 10^{-12}$ & 4 \\
\hline 28 & $\mathrm{n}-\mathrm{PrOO}+\mathrm{NO}$ & $\rightarrow \mathrm{EtCHO}+\mathrm{HO}_{2}+\mathrm{NO}_{2}$ & $2.90 \times 10^{-12} \exp \left(\frac{350}{T}\right)$ & 2 \\
\hline 29 & $\mathrm{n}-\mathrm{PrOO}+\mathrm{NO}_{3}$ & $\rightarrow \mathrm{EtCHO}+\mathrm{HO}_{2}+\mathrm{NO}_{2}$ & $2.50 \times 10^{-12}$ & 4 \\
\hline 30 & $\mathrm{i}-\mathrm{PrOO}+\mathrm{NO}$ & $\rightarrow \mathrm{Me}_{2} \mathrm{CO}+\mathrm{HO}_{2}+\mathrm{NO}_{2}$ & $2.70 \times 10^{-12} \exp \left(\frac{360}{T}\right)$ & 2 \\
\hline 31 & $\mathrm{i}-\mathrm{PrOO}+\mathrm{NO}_{3}$ & $\rightarrow \mathrm{Me}_{2} \mathrm{CO}+\mathrm{HO}_{2}+\mathrm{NO}_{2}$ & $2.50 \times 10^{-12}$ & 4 \\
\hline 32 & $\mathrm{EtCO}_{3}+\mathrm{NO}$ & $\rightarrow \mathrm{EtOO}+\mathrm{CO}_{2}+\mathrm{NO}_{2}$ & $6.70 \times 10^{-12} \exp \left(\frac{340}{T}\right)$ & 2 \\
\hline
\end{tabular}


Table 3. Continued.

\begin{tabular}{|c|c|c|c|c|}
\hline & Reactants & Products & $k$ & Reference \\
\hline 33 & $\mathrm{EtCO}_{3}+\mathrm{NO}_{3}$ & $\rightarrow \mathrm{EtOO}+\mathrm{CO}_{2}+\mathrm{NO}_{2}$ & $4.00 \times 10^{-12}$ & 4 \\
\hline 34 & $\mathrm{MeCOCH}_{2} \mathrm{OO}+\mathrm{NO}$ & $\rightarrow \mathrm{MeCO}_{3}+\mathrm{HCHO}+\mathrm{NO}_{2}$ & $2.80 \times 10^{-12} \exp \left(\frac{300}{T}\right)$ & 5 \\
\hline 35 & $\mathrm{MeCOCH}_{2} \mathrm{OO}+\mathrm{NO}_{3}$ & $\rightarrow \mathrm{MeCO}_{3}+\mathrm{HCHO}+\mathrm{NO}_{2}$ & $2.50 \times 10^{-12}$ & 4 \\
\hline 36 & $\mathrm{NO}+\mathrm{NO}_{3}$ & $\rightarrow \mathrm{NO}_{2}+\mathrm{NO}_{2}$ & $1.80 \times 10^{-11} \exp \left(\frac{110}{T}\right)$ & 2 \\
\hline 37 & $\mathrm{NO}+\mathrm{O}_{3}$ & $\rightarrow \mathrm{NO}_{2}$ & $1.40 \times 10^{-12} \exp \left(\frac{-1310}{T}\right)$ & 2 \\
\hline 38 & $\mathrm{NO}_{2}+\mathrm{O}_{3}$ & $\rightarrow \mathrm{NO}_{3}$ & $1.40 \times 10^{-13} \exp \left(\frac{-2470}{T}\right)$ & 2 \\
\hline 39 & $\mathrm{NO}_{3}+\mathrm{HCHO}$ & $\rightarrow \mathrm{HONO}_{2}+\mathrm{HO}_{2}+\mathrm{CO}$ & $2.00 \times 10^{-12} \exp \left(\frac{-2440}{T}\right)$ & 2 \\
\hline 40 & $\mathrm{NO}_{3}+\mathrm{MeCHO}$ & $\rightarrow \mathrm{HONO}_{2}+\mathrm{MeCO}_{3}$ & $1.40 \times 10^{-12} \exp \left(\frac{-1860}{T}\right)$ & 2 \\
\hline 41 & $\mathrm{NO}_{3}+\mathrm{EtCHO}$ & $\rightarrow \mathrm{HONO}_{2}+\mathrm{EtCO}_{3}$ & $3.46 \times 10^{-12} \exp \left(\frac{-1862}{T}\right)$ & 4 \\
\hline 42 & $\mathrm{NO}_{3}+\mathrm{Me}_{2} \mathrm{CO}$ & $\rightarrow \mathrm{HONO}_{2}+\mathrm{MeCOCH}_{2} \mathrm{OO}$ & $3.00 \times 10^{-17}$ & 2 \\
\hline 43 & $\mathrm{~N}_{2} \mathrm{O}_{5}+\mathrm{H}_{2} \mathrm{O}$ & $\rightarrow \mathrm{HONO}_{2}+\mathrm{HONO}_{2}$ & $2.50 \times 10^{-22}$ & 2 \\
\hline 44 & $\mathrm{O}\left({ }^{3} \mathrm{P}\right)+\mathrm{O}_{3}$ & $\rightarrow \mathrm{O}_{2}+\mathrm{O}_{2}$ & $8.00 \times 10^{-12} \exp \left(\frac{-2060}{T}\right)$ & 2 \\
\hline 45 & $\mathrm{O}\left({ }^{1} \mathrm{D}\right)+\mathrm{CH}_{4}$ & $\rightarrow \mathrm{OH}+\mathrm{MeOO}$ & $1.05 \times 10^{-10}$ & 2 \\
\hline 46 & $\mathrm{O}\left({ }^{1} \mathrm{D}\right)+\mathrm{CH}_{4}$ & $\rightarrow \mathrm{HCHO}+\mathrm{H}_{2}$ & $7.50 \times 10^{-12}$ & 2 \\
\hline 47 & $\mathrm{O}\left({ }^{1} \mathrm{D}\right)+\mathrm{CH}_{4}$ & $\rightarrow \mathrm{HCHO}+\mathrm{HO}_{2}+\mathrm{HO}_{2}$ & $3.45 \times 10^{-11}$ & 2 \\
\hline 48 & $\mathrm{O}\left({ }^{1} \mathrm{D}\right)+\mathrm{H}_{2} \mathrm{O}$ & $\rightarrow \mathrm{OH}+\mathrm{OH}$ & $2.20 \times 10^{-10}$ & 2 \\
\hline 49 & $\mathrm{O}\left({ }^{1} \mathrm{D}\right)+\mathrm{N}_{2}$ & $\rightarrow \mathrm{O}\left({ }^{3} \mathrm{P}\right)+\mathrm{N}_{2}$ & $2.10 \times 10^{-11} \exp \left(\frac{115}{T}\right)$ & 6 \\
\hline 50 & $\mathrm{O}\left({ }^{1} \mathrm{D}\right)+\mathrm{O}_{2}$ & $\rightarrow \mathrm{O}\left({ }^{3} \mathrm{P}\right)+\mathrm{O}_{2}$ & $3.20 \times 10^{-11} \exp \left(\frac{67}{T}\right)$ & 2 \\
\hline 51 & $\mathrm{OH}+\mathrm{CH}_{4}$ & $\rightarrow \mathrm{H}_{2} \mathrm{O}+\mathrm{MeOO}$ & $1.85 \times 10^{-12} \exp \left(\frac{-1690}{T}\right)$ & 2 \\
\hline 52 & $\mathrm{OH}+\mathrm{C}_{2} \mathrm{H}_{6}$ & $\rightarrow \mathrm{H}_{2} \mathrm{O}+\mathrm{EtOO}$ & $6.90 \times 10^{-12} \exp \left(\frac{-1000}{T}\right)$ & 2 \\
\hline 53 & $\mathrm{OH}+\mathrm{C}_{3} \mathrm{H}_{8}$ & $\rightarrow \mathrm{n}-\mathrm{PrOO}+\mathrm{H}_{2} \mathrm{O}$ & $7.60 \times 10^{-12} \exp \left(\frac{-585}{T}\right)$ & 2 \\
\hline 54 & $\mathrm{OH}+\mathrm{C}_{3} \mathrm{H}_{8}$ & $\rightarrow \mathrm{i}-\mathrm{PrOO}+\mathrm{H}_{2} \mathrm{O}$ & $7.60 \times 10^{-12} \exp \left(\frac{-585}{T}\right)$ & 2 \\
\hline 55 & $\mathrm{OH}+\mathrm{CO}$ & $\rightarrow \mathrm{HO}_{2}$ & $1.44 \times 10^{-13}$ & 2 \\
\hline 56 & $\mathrm{OH}+\mathrm{EtCHO}$ & $\rightarrow \mathrm{H}_{2} \mathrm{O}+\mathrm{EtCO}_{3}$ & $5.10 \times 10^{-12} \exp \left(\frac{405}{T}\right)$ & 2 \\
\hline 57 & $\mathrm{OH}+\mathrm{EtOOH}$ & $\rightarrow \mathrm{H}_{2} \mathrm{O}+\mathrm{MeCHO}+\mathrm{OH}$ & $8.01 \times 10^{-12}$ & 4 \\
\hline 58 & $\mathrm{OH}+\mathrm{EtOOH}$ & $\rightarrow \mathrm{H}_{2} \mathrm{O}+\mathrm{EtOO}$ & $1.90 \times 10^{-12} \exp \left(\frac{190}{T}\right)$ & 4 \\
\hline 59 & $\mathrm{OH}+\mathrm{H}_{2}$ & $\rightarrow \mathrm{H}_{2} \mathrm{O}+\mathrm{HO}_{2}$ & $7.70 \times 10^{-12} \exp \left(\frac{-2100}{T}\right)$ & 2 \\
\hline 60 & $\mathrm{OH}+\mathrm{H}_{2} \mathrm{O}_{2}$ & $\rightarrow \mathrm{H}_{2} \mathrm{O}+\mathrm{HO}_{2}$ & $2.90 \times 10^{-12} \exp \left(\frac{-160}{T}\right)$ & 2 \\
\hline 61 & $\mathrm{OH}+\mathrm{HCHO}$ & $\rightarrow \mathrm{H}_{2} \mathrm{O}+\mathrm{HO}_{2}+\mathrm{CO}$ & $5.40 \times 10^{-12} \exp \left(\frac{135}{T}\right)$ & 1 \\
\hline 62 & $\mathrm{OH}+\mathrm{HO}_{2}$ & $\rightarrow \mathrm{H}_{2} \mathrm{O}$ & $4.80 \times 10^{-11} \exp \left(\frac{250}{T}\right)$ & 2 \\
\hline 63 & $\mathrm{OH}+\mathrm{HO}_{2} \mathrm{NO}_{2}$ & $\rightarrow \mathrm{H}_{2} \mathrm{O}+\mathrm{NO}_{2}$ & $1.90 \times 10^{-12} \exp \left(\frac{270}{T}\right)$ & 2 \\
\hline 64 & $\mathrm{OH}+\mathrm{HO}_{2} \mathrm{NO}_{2}$ & $\rightarrow \mathrm{H}_{2} \mathrm{O}+\mathrm{NO}_{3}$ & $1.50 \times 10^{-13}$ & 2 \\
\hline 65 & $\mathrm{OH}+\mathrm{HONO}$ & $\rightarrow \mathrm{H}_{2} \mathrm{O}+\mathrm{NO}_{2}$ & $2.50 \times 10^{-12} \exp \left(\frac{260}{T}\right)$ & 2 \\
\hline 66 & $\mathrm{OH}+\mathrm{MeOOH}$ & $\rightarrow \mathrm{H}_{2} \mathrm{O}+\mathrm{HCHO}+\mathrm{OH}$ & $1.02 \times 10^{-12} \exp \left(\frac{190}{T}\right)$ & 2 \\
\hline
\end{tabular}


Table 3. Continued.

\begin{tabular}{|c|c|c|c|c|}
\hline & Reactants & Products & $k$ & Reference \\
\hline 67 & $\mathrm{OH}+\mathrm{MeOOH}$ & $\rightarrow \mathrm{H}_{2} \mathrm{O}+\mathrm{MeOO}$ & $1.89 \times 10^{-12} \exp \left(\frac{190}{T}\right)$ & 2 \\
\hline 68 & $\mathrm{OH}+\mathrm{MeONO}_{2}$ & $\rightarrow \mathrm{HCHO}+\mathrm{NO}_{2}+\mathrm{H}_{2} \mathrm{O}$ & $4.00 \times 10^{-13} \exp \left(\frac{-845}{T}\right)$ & 2 \\
\hline 69 & $\mathrm{OH}+\mathrm{Me}_{2} \mathrm{CO}$ & $\rightarrow \mathrm{H}_{2} \mathrm{O}+\mathrm{MeCOCH}_{2} \mathrm{OO}$ & $8.80 \times 10^{-12} \exp \left(\frac{-1320}{T}\right)$ & 2 \\
\hline 70 & $\mathrm{OH}+\mathrm{Me}_{2} \mathrm{CO}$ & $\rightarrow \mathrm{H}_{2} \mathrm{O}+\mathrm{MeCOCH}_{2} \mathrm{OO}$ & $1.70 \times 10^{-14} \exp \left(\frac{420}{T}\right)$ & 2 \\
\hline 71 & $\mathrm{OH}+\mathrm{MeCOCH}_{2} \mathrm{OOH}$ & $\rightarrow \mathrm{H}_{2} \mathrm{O}+\mathrm{MeCOCH}_{2} \mathrm{OO}$ & $1.90 \times 10^{-12} \exp \left(\frac{190}{T}\right)$ & 4 \\
\hline 72 & $\mathrm{OH}+\mathrm{MeCOCH}_{2} \mathrm{OOH}$ & $\rightarrow \mathrm{OH}+\mathrm{MGLY}$ & $8.39 \times 10^{-12}$ & 4 \\
\hline 73 & $\mathrm{OH}+\mathrm{MeCHO}$ & $\rightarrow \mathrm{H}_{2} \mathrm{O}+\mathrm{MeCO}_{3}$ & $4.40 \times 10^{-12} \exp \left(\frac{365}{T}\right)$ & 2 \\
\hline 74 & $\mathrm{OH}+\mathrm{NO}_{3}$ & $\rightarrow \mathrm{HO}_{2}+\mathrm{NO}_{2}$ & $2.00 \times 10^{-11}$ & 2 \\
\hline 75 & $\mathrm{OH}+\mathrm{O}_{3}$ & $\rightarrow \mathrm{HO}_{2}+\mathrm{O}_{2}$ & $1.70 \times 10^{-12} \exp \left(\frac{-940}{T}\right)$ & 2 \\
\hline 76 & $\mathrm{OH}+\mathrm{OH}$ & $\rightarrow \mathrm{H}_{2} \mathrm{O}+\mathrm{O}\left({ }^{3} \mathrm{P}\right)$ & $6.31 \times 10^{-14}\left(\frac{T}{300}\right)^{2.6} \exp \left(\frac{945}{T}\right)$ & 2 \\
\hline 77 & $\mathrm{OH}+\mathrm{PAN}$ & $\rightarrow \mathrm{HCHO}+\mathrm{NO}_{2}+\mathrm{H}_{2} \mathrm{O}$ & $3.00 \times 10^{-14}$ & 2 \\
\hline 78 & $\mathrm{OH}+\mathrm{PPAN}$ & $\rightarrow \mathrm{MeCHO}+\mathrm{NO}_{2}+\mathrm{H}_{2} \mathrm{O}$ & $1.27 \times 10^{-12}$ & 4 \\
\hline 79 & $\mathrm{OH}+\mathrm{n}-\mathrm{PrOOH}$ & $\rightarrow \mathrm{n}-\mathrm{PrOO}+\mathrm{H}_{2} \mathrm{O}$ & $1.90 \times 10^{-12} \exp \left(\frac{190}{T}\right)$ & 4 \\
\hline 80 & $\mathrm{OH}+\mathrm{n}-\mathrm{PrOOH}$ & $\rightarrow \mathrm{EtCHO}+\mathrm{H}_{2} \mathrm{O}+\mathrm{OH}$ & $1.10 \times 10^{-11}$ & 4 \\
\hline 81 & $\mathrm{OH}+\mathrm{i}-\mathrm{PrOOH}$ & $\rightarrow \mathrm{i}-\mathrm{PrOO}+\mathrm{H}_{2} \mathrm{O}$ & $1.90 \times 10^{-12} \exp \left(\frac{190}{T}\right)$ & 4 \\
\hline 82 & $\mathrm{OH}+\mathrm{i}-\mathrm{PrOOH}$ & $\rightarrow \mathrm{Me}_{2} \mathrm{CO}+\mathrm{OH}$ & $1.66 \times 10^{-11}$ & 4 \\
\hline 83 & $\mathrm{O}\left({ }^{3} \mathrm{P}\right)+\mathrm{NO}_{2}$ & $\rightarrow \mathrm{NO}+\mathrm{O}_{2}$ & $5.50 \times 10^{-12} \exp \left(\frac{188}{T}\right)$ & 2 \\
\hline 84 & $\mathrm{OH}+\mathrm{C}_{5} \mathrm{H}_{8}$ & $\rightarrow \mathrm{ISO}_{2}$ & $2.70 \times 10^{-11} \exp \left(\frac{390}{T}\right)$ & 2 \\
\hline $85^{*}$ & $\mathrm{OH}+\mathrm{C}_{5} \mathrm{H}_{8}$ & $\rightarrow \mathrm{MACR}+\mathrm{HCHO}+\mathrm{MACRO}_{2}+\mathrm{MeCO}_{3}$ & $3.33 \times 10^{-15} \exp \left(\frac{-1995}{T}\right)$ & 2 \\
\hline $86^{*}$ & $\mathrm{OH}+\mathrm{C}_{5} \mathrm{H}_{8}$ & $\rightarrow \mathrm{MeOO}+\mathrm{HCOOH}+\mathrm{CO}+\mathrm{H}_{2} \mathrm{O}_{2}$ & $3.33 \times 10^{-15} \exp \left(\frac{-1995}{T}\right)$ & 2 \\
\hline $87^{*}$ & $\mathrm{OH}+\mathrm{C}_{5} \mathrm{H}_{8}$ & $\rightarrow \mathrm{HO}_{2}+\mathrm{OH}$ & $3.33 \times 10^{-15} \exp \left(\frac{-1995}{T}\right)$ & 2 \\
\hline 88 & $\mathrm{NO}_{3}+\mathrm{C}_{5} \mathrm{H}_{8}$ & $\rightarrow$ ISON & $3.15 \times 10^{-12} \exp \left(\frac{-450}{T}\right)$ & 2 \\
\hline 89 & $\mathrm{NO}+\mathrm{ISO}_{2}$ & $\rightarrow \mathrm{NO}_{2}+\mathrm{MACR}+\mathrm{HCHO}+\mathrm{HO}_{2}$ & $2.43 \times 10^{-12} \exp \left(\frac{360}{T}\right)$ & 4,7 \\
\hline 90 & $\mathrm{NO}+\mathrm{ISO}_{2}$ & $\rightarrow$ ISON & $1.12 \times 10^{-13} \exp \left(\frac{360}{T}\right)$ & 4,7 \\
\hline 91 & $\mathrm{HO}_{2}+\mathrm{ISO}_{2}$ & $\rightarrow$ ISOOH & $2.05 \times 10^{-13} \exp \left(\frac{1300}{T}\right)$ & 4,7 \\
\hline 92 & $\mathrm{ISO}_{2}+\mathrm{ISO}_{2}$ & $\rightarrow \mathrm{MACR}+\mathrm{MACR}+\mathrm{HCHO}+\mathrm{HO}_{2}$ & $2.00 \times 10^{-12}$ & 7 \\
\hline 93 & $\mathrm{OH}+\mathrm{ISOOH}$ & $\rightarrow \mathrm{MACR}+\mathrm{OH}$ & $1.00 \times 10^{-10}$ & 7 \\
\hline 94 & $\mathrm{OH}+\mathrm{ISON}$ & $\rightarrow$ HACET + NALD & $1.30 \times 10^{-11}$ & 7 \\
\hline 95 & $\mathrm{OH}+\mathrm{MACR}$ & $\rightarrow \mathrm{MACRO}_{2}$ & $1.30 \times 10^{-12} \exp \left(\frac{610}{T}\right)$ & 2 \\
\hline 96 & $\mathrm{OH}+\mathrm{MACR}$ & $\rightarrow \mathrm{MACRO}_{2}$ & $4.00 \times 10^{-12} \exp \left(\frac{380}{T}\right)$ & 2 \\
\hline $97^{*}$ & $\mathrm{O}_{3}+\mathrm{MACR}$ & $\rightarrow \mathrm{MGLY}+\mathrm{HCOOH}+\mathrm{HO}_{2}+\mathrm{CO}$ & $2.13 \times 10^{-16} \exp \left(\frac{-1520}{T}\right)$ & 2 \\
\hline $98^{*}$ & $\mathrm{O}_{3}+\mathrm{MACR}$ & $\rightarrow \mathrm{OH}+\mathrm{MeCO}_{3}$ & $2.13 \times 10^{-16} \exp \left(\frac{-1520}{T}\right)$ & 2 \\
\hline $99^{*}$ & $\mathrm{O}_{3}+\mathrm{MACR}$ & $\rightarrow \mathrm{MGLY}+\mathrm{HCOOH}+\mathrm{HO}_{2}+\mathrm{CO}$ & $3.50 \times 10^{-16} \exp \left(\frac{-2100}{T}\right)$ & 2 \\
\hline $100^{*}$ & $\mathrm{O}_{3}+\mathrm{MACR}$ & $\rightarrow \mathrm{OH}+\mathrm{MeCO}_{3}$ & $3.50 \times 10^{-16} \exp \left(\frac{-2100}{T}\right)$ & 2 \\
\hline
\end{tabular}


Table 3. Continued.

\begin{tabular}{|c|c|c|c|c|}
\hline & Reactants & Products & $k$ & Reference \\
\hline $101^{*}$ & $\mathrm{NO}+\mathrm{MACRO}_{2}$ & $\rightarrow \mathrm{NO}_{2}+\mathrm{MeCO}_{3}+\mathrm{HACET}+\mathrm{CO}$ & $1.27 \times 10^{-12} \exp \left(\frac{360}{T}\right)$ & 4,7 \\
\hline $102^{*}$ & $\mathrm{NO}+\mathrm{MACRO}_{2}$ & $\rightarrow \mathrm{MGLY}+\mathrm{HCHO}+\mathrm{HO}_{2}$ & $1.27 \times 10^{-12} \exp \left(\frac{360}{T}\right)$ & 4,7 \\
\hline 103 & $\mathrm{HO}_{2}+\mathrm{MACRO}_{2}$ & $\rightarrow \mathrm{MACROOH}$ & $1.83 \times 10^{-13} \exp \left(\frac{1300}{T}\right)$ & 4,7 \\
\hline $104^{*}$ & $\mathrm{MACRO}_{2}+\mathrm{MACRO}_{2}$ & $\rightarrow \mathrm{HACET}+\mathrm{MGLY}+\mathrm{HCHO}+\mathrm{CO}$ & $1.00 \times 10^{-12}$ & 4,7 \\
\hline $105^{*}$ & $\mathrm{MACRO}_{2}+\mathrm{MACRO}_{2}$ & $\rightarrow \mathrm{HO}_{2}$ & $1.00 \times 10^{-12}$ & 4,7 \\
\hline 106 & $\mathrm{OH}+\mathrm{MPAN}$ & $\rightarrow \mathrm{HACET}+\mathrm{NO}_{2}$ & $2.90 \times 10^{-11}$ & 2 \\
\hline 107 & $\mathrm{OH}+\mathrm{MACROOH}$ & $\rightarrow \mathrm{MACRO}_{2}$ & $3.00 \times 10^{-11}$ & 7 \\
\hline 108 & $\mathrm{OH}+\mathrm{HACET}$ & $\rightarrow \mathrm{MGLY}+\mathrm{HO}_{2}$ & $3.00 \times 10^{-12}$ & 2,7 \\
\hline 109 & $\mathrm{OH}+\mathrm{MGLY}$ & $\rightarrow \mathrm{MeCO}_{3}+\mathrm{CO}$ & $1.50 \times 10^{-11}$ & 2,7 \\
\hline 110 & $\mathrm{NO}_{3}+\mathrm{MGLY}$ & $\rightarrow \mathrm{MeCO}_{3}+\mathrm{CO}+\mathrm{HONO}_{2}$ & $3.46 \times 10^{-12} \exp \left(\frac{-1860}{T}\right)$ & 4 \\
\hline 111 & $\mathrm{OH}+\mathrm{NALD}$ & $\rightarrow \mathrm{HCHO}+\mathrm{CO}+\mathrm{NO}_{2}$ & $4.40 \times 10^{-12} \exp \left(\frac{365}{T}\right)$ & 2,7 \\
\hline 112 & $\mathrm{OH}+\mathrm{MeCO}_{3} \mathrm{H}$ & $\rightarrow \mathrm{MeCO}_{3}$ & $3.70 \times 10^{-12}$ & 4,7 \\
\hline 113 & $\mathrm{OH}+\mathrm{MeCO}_{2} \mathrm{H}$ & $\rightarrow \mathrm{MeOO}$ & $4.00 \times 10^{-13} \exp \left(\frac{200}{T}\right)$ & 7 \\
\hline 114 & $\mathrm{OH}+\mathrm{HCOOH}$ & $\rightarrow \mathrm{HO}_{2}$ & $4.50 \times 10^{-13}$ & 2 \\
\hline 115 & $\mathrm{MeOH}+\mathrm{OH}$ & $\rightarrow \mathrm{HCHO}+\mathrm{HO}_{2}$ & $2.85 \times 10^{-12} \exp \left(\frac{-345}{T}\right)$ & 3 \\
\hline 116 & $\mathrm{OH}+\mathrm{C}_{10} \mathrm{H}_{16}$ & $\rightarrow \mathrm{TERPO}_{2}$ & $1.20 \times 10^{-11} \exp \left(\frac{444}{T}\right)$ & 8 \\
\hline 117 & $\mathrm{O}_{3}+\mathrm{C}_{10} \mathrm{H}_{16}$ & $\rightarrow \mathrm{OH}+\mathrm{MEK}+\mathrm{HO}_{2}$ & $1.00 \times 10^{-15} \exp \left(\frac{-732}{T}\right)$ & 8 \\
\hline 118 & $\mathrm{NO}_{3}+\mathrm{C}_{10} \mathrm{H}_{16}$ & $\rightarrow \mathrm{ISON}+\mathrm{MACR}$ & $1.20 \times 10^{-12} \exp \left(\frac{490}{T}\right)$ & 8 \\
\hline $119^{*}$ & $\mathrm{NO}+\mathrm{TERPO}_{2}$ & $\rightarrow \mathrm{Me}_{2} \mathrm{CO}+\mathrm{HO}_{2}+\mathrm{NO}_{2}$ & $2.10 \times 10^{-12} \exp \left(\frac{180}{T}\right)$ & 8 \\
\hline $120^{*}$ & $\mathrm{NO}+\mathrm{TERPO}_{2}$ & $\rightarrow \mathrm{MACR}+\mathrm{MACR}$ & $2.10 \times 10^{-12} \exp \left(\frac{180}{T}\right)$ & 8 \\
\hline $121^{*}$ & $\mathrm{HO}_{2}+\mathrm{TERPO}_{2}$ & $\rightarrow$ TERPOOH & $7.50 \times 10^{-13} \exp \left(\frac{700}{T}\right)$ & 8 \\
\hline $122^{*}$ & $\mathrm{OH}+\mathrm{TERPOOH}$ & $\rightarrow \mathrm{TERPO}_{2}$ & $3.80 \times 10^{-12} \exp \left(\frac{200}{T}\right)$ & 8 \\
\hline 123 & $\mathrm{C}_{4} \mathrm{H}_{10}+\mathrm{OH}$ & $\rightarrow \mathrm{BtOO}+\mathrm{H}_{2} \mathrm{O}$ & $9.10 \times 10^{-12} \exp \left(\frac{-405}{T}\right)$ & 3 \\
\hline $124^{*}$ & $\mathrm{BtOO}+\mathrm{NO}$ & $\rightarrow \mathrm{NO}_{2}+\mathrm{MEK}+\mathrm{HO}_{2}+\mathrm{EtOO}$ & $1.27 \times 10^{-12} \exp \left(\frac{360}{T}\right)$ & 4 \\
\hline $125^{*}$ & $\mathrm{BtOO}+\mathrm{NO}$ & $\rightarrow \mathrm{ONIT}+\mathrm{MeCHO}$ & $1.27 \times 10^{-12} \exp \left(\frac{360}{T}\right)$ & 4 \\
\hline 126 & $\mathrm{BtOO}+\mathrm{HO}_{2}$ & $\rightarrow \mathrm{BtOOH}$ & $1.82 \times 10^{-13} \exp \left(\frac{1300}{T}\right)$ & 4 \\
\hline $127^{*}$ & $\mathrm{BtOO}+\mathrm{MeOO}$ & $\rightarrow \mathrm{MEK}+\mathrm{HCHO}+\mathrm{HO}_{2}+\mathrm{MeCHO}$ & $1.25 \times 10^{-13}$ & 4 \\
\hline $128^{*}$ & $\mathrm{BtOO}+\mathrm{MeOO}$ & $\rightarrow \mathrm{MeOH}+\mathrm{EtOO}$ & $1.25 \times 10^{-13}$ & 4 \\
\hline $129^{*}$ & $\mathrm{BtOOH}+\mathrm{OH}$ & $\rightarrow \mathrm{BtOO}+\mathrm{MEK}+\mathrm{OH}+\mathrm{H}_{2} \mathrm{O}$ & $1.90 \times 10^{-12} \exp \left(\frac{190}{T}\right)$ & 4 \\
\hline 130 & $\mathrm{MEK}+\mathrm{OH}$ & $\rightarrow$ MEKOO & $1.30 \times 10^{-12} \exp \left(\frac{-25}{T}\right)$ & 3 \\
\hline 131 & $\mathrm{MEKOO}+\mathrm{NO}$ & $\rightarrow \mathrm{MeCHO}+\mathrm{MeCO}_{3}+\mathrm{NO}_{2}+\mathrm{ONIT}$ & $2.54 \times 10^{-12} \exp \left(\frac{360}{T}\right)$ & 4 \\
\hline 132 & $\mathrm{MEKOO}+\mathrm{HO}_{2}$ & $\rightarrow \mathrm{MEKOOH}$ & $1.82 \times 10^{-13} \exp \left(\frac{1300}{T}\right)$ & 4 \\
\hline 133 & $\mathrm{MEKOOH}+\mathrm{OH}$ & $\rightarrow \mathrm{MeCOCOMe}+\mathrm{OH}+\mathrm{OH}$ & $1.90 \times 10^{-12} \exp \left(\frac{190}{T}\right)$ & 4 \\
\hline 134 & $\mathrm{ONIT}+\mathrm{OH}$ & $\rightarrow \mathrm{MEK}+\mathrm{NO}_{2}+\mathrm{H}_{2} \mathrm{O}$ & $1.60 \times 10^{-12}$ & 3 \\
\hline
\end{tabular}


Table 3. Continued.

\begin{tabular}{|c|c|c|c|c|}
\hline & Reactants & Products & $k$ & Reference \\
\hline $135^{*}$ & $\mathrm{C}_{2} \mathrm{H}_{4}+\mathrm{O}_{3}$ & $\rightarrow \mathrm{HCHO}+\mathrm{HO}_{2}+\mathrm{OH}+\mathrm{CO}$ & $4.55 \times 10^{-15} \exp \left(\frac{-2580}{T}\right)$ & 3 \\
\hline $136^{*}$ & $\mathrm{C}_{2} \mathrm{H}_{4}+\mathrm{O}_{3}$ & $\rightarrow \mathrm{H}_{2}+\mathrm{CO}_{2}+\mathrm{HCOOH}$ & $4.55 \times 10^{-15} \exp \left(\frac{-2580}{T}\right)$ & 3 \\
\hline $137^{*}$ & $\mathrm{C}_{3} \mathrm{H}_{6}+\mathrm{O}_{3}$ & $\rightarrow \mathrm{HCHO}+\mathrm{MeCHO}+\mathrm{OH}+\mathrm{HO}_{2}$ & $1.83 \times 10^{-15} \exp \left(\frac{-1880}{T}\right)$ & 3 \\
\hline $138^{*}$ & $\mathrm{C}_{3} \mathrm{H}_{6}+\mathrm{O}_{3}$ & $\rightarrow \mathrm{EtOO}+\mathrm{MGLY}+\mathrm{CH}_{4}+\mathrm{CO}$ & $1.83 \times 10^{-15} \exp \left(\frac{-1880}{T}\right)$ & 3 \\
\hline $139^{*}$ & $\mathrm{C}_{3} \mathrm{H}_{6}+\mathrm{O}_{3}$ & $\rightarrow \mathrm{MeOH}+\mathrm{MeOO}+\mathrm{HCOOH}$ & $1.83 \times 10^{-15} \exp \left(\frac{-1880}{T}\right)$ & 3 \\
\hline $140^{*}$ & $\mathrm{C}_{3} \mathrm{H}_{6}+\mathrm{NO}_{3}$ & $\rightarrow$ ONIT & $4.60 \times 10^{-13} \exp \left(\frac{-1155}{T}\right)$ & 3 \\
\hline $141^{*}$ & PrpeOO $+\mathrm{NO}$ & $\rightarrow \mathrm{MeCHO}+\mathrm{HCHO}+\mathrm{HO}_{2}+\mathrm{NO}_{2}$ & $1.27 \times 10^{-12} \exp \left(\frac{360}{T}\right)$ & 4 \\
\hline $142^{*}$ & PrpeOO + NO & $\rightarrow$ ONIT & $1.27 \times 10^{-12} \exp \left(\frac{360}{T}\right)$ & 4 \\
\hline 143 & $\mathrm{PrpeOO}+\mathrm{HO}_{2}$ & $\rightarrow$ PrpeOOH & $1.50 \times 10^{-13} \exp \left(\frac{1300}{T}\right)$ & 4 \\
\hline 144 & PrpeOOH + OH & $\rightarrow$ PrpeOO $+\mathrm{H}_{2} \mathrm{O}$ & $1.90 \times 10^{-12} \exp \left(\frac{190}{T}\right)$ & 4 \\
\hline 145 & PrpeOOH + OH & $\rightarrow \mathrm{HACET}+\mathrm{OH}$ & $2.44 \times 10^{-11}$ & 4 \\
\hline $146^{*}$ & $\mathrm{AROM}+\mathrm{OH}$ & $\rightarrow \mathrm{AROMO}_{2}+\mathrm{HO}_{2}$ & $1.81 \times 10^{-12} \exp \left(\frac{338}{T}\right)$ & 9 \\
\hline $147^{*}$ & $\mathrm{AROMO}_{2}+\mathrm{NO}$ & $\rightarrow \mathrm{MGLY}+\mathrm{NO}_{2}+\mathrm{MeCO}_{3}+\mathrm{CO}$ & $1.35 \times 10^{-12} \exp \left(\frac{360}{T}\right)$ & 9 \\
\hline $148^{*}$ & $\mathrm{AROMO}_{2}+\mathrm{NO}$ & $\rightarrow \mathrm{HO}_{2}$ & $1.35 \times 10^{-12} \exp \left(\frac{360}{T}\right)$ & 9 \\
\hline $149^{*}$ & $\mathrm{AROMO}_{2}+\mathrm{NO}_{3}$ & $\rightarrow \mathrm{MGLY}+\mathrm{NO}_{2}+\mathrm{MeCO}_{3}+\mathrm{CO}$ & $1.20 \times 10^{-12}$ & 9 \\
\hline $150^{*}$ & $\mathrm{AROMO}_{2}+\mathrm{NO}_{3}$ & $\rightarrow \mathrm{HO}_{2}$ & $1.20 \times 10^{-12}$ & 9 \\
\hline $151^{*}$ & $\mathrm{AROMO}_{2}+\mathrm{HO}_{2}$ & $\rightarrow$ AROMOOH & $1.90 \times 10^{-13} \exp \left(\frac{-1300}{T}\right)$ & 9 \\
\hline $152^{*}$ & $\mathrm{AROMO}_{2}+\mathrm{MeOO}$ & $\rightarrow \mathrm{MGLY}+\mathrm{CO}+\mathrm{MeCO}_{3}+\mathrm{MeOH}$ & $1.15 \times 10^{-13}$ & 9 \\
\hline $153^{*}$ & $\mathrm{AROMO}_{2}+\mathrm{MeOO}$ & $\rightarrow \mathrm{HO}_{2}+\mathrm{HCHO}$ & $1.15 \times 10^{-13}$ & 9 \\
\hline $154^{*}$ & $\mathrm{AROMOOH}+\mathrm{OH}$ & $\rightarrow \mathrm{AROMO}_{2}$ & $1.90 \times 10^{-12} \exp \left(\frac{190}{T}\right)$ & 9 \\
\hline $155^{*}$ & $\mathrm{AROMOOH}+\mathrm{OH}$ & $\rightarrow \mathrm{OH}+\mathrm{H}_{2} \mathrm{O}$ & $4.61 \times 10^{-18} \exp \left(\frac{253}{T}\right)$ & 9 \\
\hline $156^{*}$ & $\mathrm{AROMOOH}+\mathrm{OH}$ & $\rightarrow \mathrm{MeCO}_{3}+\mathrm{CO}+\mathrm{HO}_{2}+\mathrm{OH}$ & $4.19 \times 10^{-17} \exp \left(\frac{696}{T}\right)$ & 9 \\
\hline 157 & $\mathrm{HO}_{2}+\mathrm{O}_{3} \mathrm{~S}$ & $\rightarrow \mathrm{HO}_{2}+\mathrm{O}_{2}$ & $2.03 \times 10^{-16}\left(\frac{T}{300}\right)^{4.57} \exp \left(\frac{693}{T}\right)$ & 2 \\
\hline 158 & $\mathrm{OH}+\mathrm{O}_{3} \mathrm{~S}$ & $\rightarrow \mathrm{OH}+\mathrm{O}_{2}$ & $1.70 \times 10^{-12} \exp \left(\frac{-940}{T}\right)$ & 2 \\
\hline 159 & $\mathrm{O}\left({ }^{1} \mathrm{D}\right) \mathrm{S}+\mathrm{H}_{2} \mathrm{O}$ & $\rightarrow \mathrm{H}_{2} \mathrm{O}$ & $2.20 \times 10^{-10}$ & 2 \\
\hline 160 & $\mathrm{O}\left({ }^{1} \mathrm{D}\right) \mathrm{S}+\mathrm{N}_{2}$ & $\rightarrow \mathrm{O}\left({ }^{3} \mathrm{P}\right) \mathrm{S}+\mathrm{N}_{2}$ & $2.10 \times 10^{-11} \exp \left(\frac{115}{T}\right)$ & 6 \\
\hline 161 & $\mathrm{O}\left({ }^{1} \mathrm{D}\right) \mathrm{S}+\mathrm{O}_{2}$ & $\rightarrow \mathrm{O}\left({ }^{3} \mathrm{P}\right) \mathrm{S}+\mathrm{O}_{2}$ & $3.20 \times 10^{-11} \exp \left(\frac{67}{T}\right)$ & 2 \\
\hline
\end{tabular}

* Reactions are split between multiple lines.

tochemical ozone creation potentials of 100 and 105 for ethene and propene, respectively, compared to 8.8 and 18.3 for ethane and propane, respectively (Saunders et al., 2003). The lumped monoterpene compound $\left(\mathrm{C}_{1} \mathrm{OH}_{1} 6\right)$ is treated as $\alpha$-pinene, with emissions made up of the sum of $\alpha$-pinene, $\beta$-pinene, limonene, myrcene, ocimene, sabinene, and $\delta$-3carene. Including monoterpene chemistry adds six bimolec- ular reactions (R116-R122, Table 3) and one photolysis reaction (R26-27, Table 5) to the TOMCAT extended scheme. Monoterpenes are the second largest biogenic source of VOCs, after isoprene, and play an important role in $\mathrm{OH}$, $\mathrm{NO}_{3}, \mathrm{O}_{3}$ and aerosol chemistry (e.g. Atkinson and Arey, 2003; Fuentes et al., 2000). Accounting for monoterpenes 
Table 4. TOMCAT gas-phase termolecular and thermal decomposition reactions. Rate constant $k=\left(\frac{k_{0}[M]}{1+k_{0}[M] / k_{\infty}}\right) F_{\mathrm{c}}{ }^{\left(1+\left[\log \frac{k_{0}[M]}{k_{\infty}}\right]^{2}\right)^{-1}}$ where $k_{0}$ is the low pressure limit, $k_{\infty}$ is the high pressure limit and $M$ is the number density in molecules $\mathrm{cm}^{-3} . F_{\mathrm{c}}=f$ when $f<1$ else $F_{\mathrm{c}}=\exp (-T / f)$. Low pressure limit $k_{0}=k_{1}\left(\frac{T}{300}\right)^{\alpha_{1}} \exp \left(\frac{-\beta_{1}}{T}\right)$ and high pressure limit $k_{\infty}=k_{2}\left(\frac{T}{300}\right)^{\alpha_{2}} \exp \left(\frac{-\beta_{2}}{T}\right)$. Reaction rate references 1: Atkinson et al. (2005), 2: MCM (2004), 3: Pöschl et al. (2000), 4: Atkinson et al. (2006a).

\begin{tabular}{|c|c|c|c|c|c|c|c|c|c|c|}
\hline & Reactants & Products & $f$ & $k_{1}$ & $\alpha_{1}$ & $\beta_{1}$ & $k_{2}$ & $\alpha_{2}$ & $\beta_{2}$ & Reference \\
\hline $1^{\mathrm{a}}$ & $\mathrm{HO}_{2}+\mathrm{HO}_{2}+\mathrm{M}$ & $\rightarrow \mathrm{H}_{2} \mathrm{O}_{2}+\mathrm{O}_{2}+\mathrm{M}$ & 0.00 & $1.90 \times 10^{-33}$ & 0.00 & -980.0 & $0.00 \times 10^{+00}$ & 0.00 & $0.0^{1}$ & 1 \\
\hline 2 & $\mathrm{HO}_{2}+\mathrm{NO}_{2}+\mathrm{M}$ & $\rightarrow \mathrm{HO}_{2} \mathrm{NO}_{2}+\mathrm{M}$ & 0.60 & $1.80 \times 10^{-31}$ & -3.20 & 0.0 & $4.70 \times 10^{-12}$ & 0.00 & 0.0 & 1 \\
\hline 3 & $\mathrm{HO}_{2} \mathrm{NO}_{2}+\mathrm{M}$ & $\rightarrow \mathrm{HO}_{2}+\mathrm{NO}_{2}+\mathrm{M}$ & 0.60 & $4.10 \times 10^{-05}$ & 0.00 & 10650.0 & $4.80 \times 10^{+15}$ & 0.00 & 11170.0 & 1 \\
\hline 4 & $\mathrm{MeCO}_{3}+\mathrm{NO}_{2}+\mathrm{M}$ & $\rightarrow \mathrm{PAN}+\mathrm{M}$ & 0.30 & $2.70 \times 10^{-28}$ & -7.10 & 0.0 & $1.20 \times 10^{-11}$ & -0.90 & 0.0 & 1 \\
\hline 5 & $\mathrm{PAN}+\mathrm{M}$ & $\rightarrow \mathrm{MeCO}_{3}+\mathrm{NO}_{2}+\mathrm{M}$ & 0.30 & $4.90 \times 10^{-03}$ & 0.00 & 12100.0 & $5.40 \times 10^{+16}$ & 0.00 & 13830.0 & 1 \\
\hline 6 & $\mathrm{~N}_{2} \mathrm{O}_{5}+\mathrm{M}$ & $\rightarrow \mathrm{NO}_{2}+\mathrm{NO}_{3}+\mathrm{M}$ & 0.35 & $1.30 \times 10^{-03}$ & -3.50 & 11000.0 & $9.70 \times 10^{+14}$ & 0.10 & 11080.0 & 1 \\
\hline 7 & $\mathrm{NO}_{2}+\mathrm{NO}_{3}+\mathrm{M}$ & $\rightarrow \mathrm{N}_{2} \mathrm{O}_{5}+\mathrm{M}$ & 0.35 & $3.60 \times 10^{-30}$ & -4.10 & 0.0 & $1.90 \times 10^{-12}$ & 0.20 & 0.0 & 1 \\
\hline 8 & $\mathrm{O}\left({ }^{3} \mathrm{P}\right)+\mathrm{O}_{2}+\mathrm{M}$ & $\rightarrow \mathrm{O}_{3}+\mathrm{M}$ & 0.00 & $5.70 \times 10^{-34}$ & -2.60 & 0.0 & $0.00 \times 10^{+00}$ & 0.00 & 0.0 & 1 \\
\hline 9 & $\mathrm{OH}+\mathrm{NO}+\mathrm{M}$ & $\rightarrow \mathrm{HONO}+\mathrm{M}$ & 1420.00 & $7.40 \times 10^{-31}$ & -2.40 & 0.0 & $3.30 \times 10^{-11}$ & -0.30 & 0.0 & 1 \\
\hline 10 & $\mathrm{OH}+\mathrm{NO}_{2}+\mathrm{M}$ & $\rightarrow \mathrm{HONO}_{2}+\mathrm{M}$ & 0.40 & $3.30 \times 10^{-30}$ & -3.00 & 0.0 & $4.10 \times 10^{-11}$ & 0.00 & 0.0 & 1 \\
\hline 11 & $\mathrm{OH}+\mathrm{OH}+\mathrm{M}$ & $\rightarrow \mathrm{H}_{2} \mathrm{O}_{2}+\mathrm{M}$ & 0.50 & $6.90 \times 10^{-31}$ & -0.80 & 0.0 & $2.60 \times 10^{-11}$ & 0.00 & 0.0 & 1 \\
\hline 12 & $\mathrm{EtCO}_{3}+\mathrm{NO}_{2}+\mathrm{M}$ & $\rightarrow$ PPAN + M & 0.30 & $2.70 \times 10^{-28}$ & -7.10 & 0.0 & $1.20 \times 10^{-11}$ & -0.90 & 0.0 & 2 \\
\hline 13 & $\mathrm{PPAN}+\mathrm{M}$ & $\rightarrow \mathrm{EtCO}_{3}+\mathrm{NO}_{2}+\mathrm{M}$ & 0.36 & $1.70 \times 10^{-03}$ & 0.00 & 11280.0 & $8.30 \times 10^{+16}$ & 0.00 & 13940.0 & 1 \\
\hline 14 & $\mathrm{MACRO}_{2}+\mathrm{NO}_{2}+\mathrm{M}$ & $\rightarrow \mathrm{MPAN}+\mathrm{M}$ & 0.30 & $2.70 \times 10^{-28}$ & 0.00 & 11280.0 & $8.30 \times 10^{+16}$ & 0.00 & 13940.0 & 3 \\
\hline 15 & $\mathrm{MPAN}+\mathrm{M}$ & $\rightarrow \mathrm{MACRO}_{2}+\mathrm{NO}_{2}+\mathrm{M}$ & 0.30 & $4.90 \times 10^{-03}$ & 0.00 & 12100.0 & $5.40 \times 10^{+16}$ & 0.00 & 13830.0 & 3 \\
\hline 16 & $\mathrm{O}\left({ }^{3} \mathrm{P}\right)+\mathrm{O}_{2}+\mathrm{M}$ & $\rightarrow \mathrm{O}_{3}+\mathrm{M}$ & 0.00 & $5.70 \times 10^{-34}$ & -2.60 & 0.0 & $0.00 \times 10^{+00}$ & 0.00 & 0.0 & 1 \\
\hline $17^{\mathrm{b}}$ & $\mathrm{C}_{2} \mathrm{H}_{4}+\mathrm{OH}+\mathrm{M}$ & $\rightarrow$ PrpeOO $+\mathrm{M}$ & 0.48 & $2.87 \times 10^{-29}$ & -3.10 & 0.0 & $3.00 \times 10^{-12}$ & -0.85 & 0.0 & 4 \\
\hline $18^{\mathrm{b}}$ & $\mathrm{C}_{2} \mathrm{H}_{4}+\mathrm{OH}+\mathrm{M}$ & $\rightarrow$ PrpeOO $+\mathrm{M}$ & 0.48 & $2.87 \times 10^{-29}$ & -3.10 & 0.0 & $3.00 \times 10^{-12}$ & -0.85 & 0.0 & 4 \\
\hline $19^{\mathrm{b}}$ & $\mathrm{C}_{2} \mathrm{H}_{4}+\mathrm{OH}+\mathrm{M}$ & $\rightarrow$ & 0.48 & $2.87 \times 10^{-29}$ & -3.10 & 0.0 & $3.00 \times 10^{-12}$ & -0.85 & 0.0 & 4 \\
\hline 20 & $\mathrm{C}_{3} \mathrm{H}_{6}+\mathrm{OH}+\mathrm{M}$ & $\rightarrow$ PrpeOO $+\mathrm{M}$ & 0.50 & $8.00 \times 10^{-27}$ & -3.50 & 0.0 & $3.00 \times 10^{-11}$ & -1.00 & 0.0 & 4 \\
\hline
\end{tabular}

${ }^{a}$ Reaction rate is dependent on $\mathrm{H}_{2} \mathrm{O}$ so $k$ is weighted by factor of $1+1.4 \times 10^{-21}\left[\mathrm{H}_{2} \mathrm{O}\right] \exp (2200 / T)$, where $\left[\mathrm{H}_{2} \mathrm{O}\right]$ is in molecules $\mathrm{cm}^{-3}$.

$\mathrm{b}$ Reactions are split between multiple lines.

also allows the coupling of the atmospheric chemistry model, TOMCAT, to the aerosol model, GLOMAP.

Photolysis rates are calculated online at each chemical time step based on the two-stream method of Hough (1988), which considers both direct and scattered radiation. The scheme has total of 203 wavelength intervals from 120 to $850 \mathrm{~nm}$, though only wavelengths above $175 \mathrm{~nm}$ are used for stratosphere-troposphere studies. These wavelength intervals are the same as those employed in the TOMCAT stratospheric chemistry scheme (Chipperfield et al., 2015; Sukhodolov et al., 2016). The top of the atmosphere solar flux spectrum is fixed in time and there is no account of, for example, the 11-year solar cycle in the standard model. This photolysis scheme is coupled with the TOMCAT model by using the model temperature and ozone concentration profiles. The scheme is also supplied with surface albedo, aerosol concentrations and monthly mean climatological cloud fields. This scheme was first used in this manner by Arnold et al. (2005). Previously, an offline approach was used where photolysis rates were calculated offline and then read in to the model (e.g. Law et al., 1998). Where possible, photochemical data are taken from Sander et al. (2011) for species which are also relevant for the stratosphere. Otherwise, photochemical data are generally taken from IUPAC (Atkinson et al., 2004b, 2006b). The UV absorption cross sections for methyl hydroperoxide (MeOOH), which are from the Jet Propulsion Laboratory (JPL) (Sander et al., 2006), are used for the hydroperoxides produced from the oxidation of butane $(\mathrm{BtOOH})$, toluene $(\mathrm{AROMOOH})$ and monoterpene (TERPOOH). For the photolysis of ONIT, which represents organic nitrates produced from higher alkanes (currently only butane), cross sections for methyl nitrate are used based on IUPAC recommendations (Atkinson et al., 2006b). ONIT can be an important reservoir of reactive nitrogen (von Kuhlmann, 2001). Stratospheric concentrations of $\mathrm{O}_{3}$ and $\mathrm{NO}_{y}$ calculated offline by the 2-D Cambridge model (Law and Pyle, 1993) are read in by TOMCAT in the absence of stratospheric chemistry. Hydrogen, carbon dioxide, oxygen and nitrogen are kept at fixed global mean volume mixing ratios in the model. Water vapour is calculated from the specific humidity field read in from the meteorological input data.

Heterogeneous chemistry is known to affect the global concentrations of $\mathrm{O}_{3}, \mathrm{OH}$ and $\mathrm{NO}_{x}$ in the troposphere (Ja$\mathrm{cob}, 2000)$. One important reaction is that of dinitrogen pentoxide $\left(\mathrm{N}_{2} \mathrm{O}_{5}\right)$ with water $\left(\mathrm{H}_{2} \mathrm{O}\right)$ on the surface of aerosols to form nitric acid $\left(\mathrm{HNO}_{3}\right) . \mathrm{HNO}_{3}$ is highly soluble and is therefore efficiently lost through wet deposition, making this an important loss channel for $\mathrm{NO}_{x}$ from the atmosphere. This is important in the troposphere when there is no sunlight, allowing time for the formation of $\mathrm{N}_{2} \mathrm{O}_{5}$. TOMCAT can be run coupled to the GLOMAP aerosol module (Mann et al., 2010), which can then calculate the available aerosol surface area for use in the heterogeneous chemistry calculation (e.g. 
Table 5. TOMCAT photolysis reactions.

\begin{tabular}{|c|c|c|}
\hline Reaction & Reactants & Products \\
\hline 1 & $\mathrm{EtOOH}+h v$ & $\rightarrow \mathrm{MeCHO}+\mathrm{HO}_{2}+\mathrm{OH}$ \\
\hline 2 & $\mathrm{H}_{2} \mathrm{O}_{2}+h v$ & $\rightarrow \mathrm{OH}+\mathrm{OH}$ \\
\hline $3 a$ & $\mathrm{HCHO}+h v$ & $\rightarrow \mathrm{HO}_{2}+\mathrm{HO}_{2}+\mathrm{CO}$ \\
\hline $3 b$ & $\mathrm{HCHO}+h v$ & $\rightarrow \mathrm{H}_{2}+\mathrm{CO}$ \\
\hline 5 & $\mathrm{HO}_{2} \mathrm{NO}_{2}+h v$ & $\rightarrow \mathrm{HO}_{2}+\mathrm{NO}_{2}$ \\
\hline 6 & $\mathrm{HONO}_{2}+h v$ & $\rightarrow \mathrm{OH}+\mathrm{NO}_{2}$ \\
\hline $7 \mathrm{a}$ & $\mathrm{MeCHO}+h v$ & $\rightarrow \mathrm{MeOO}+\mathrm{HO}_{2}+\mathrm{CO}$ \\
\hline $7 b$ & $\mathrm{MeCHO}+h v$ & $\rightarrow \mathrm{CH} 4+\mathrm{CO}$ \\
\hline 9 & $\mathrm{MeOOH}+h v$ & $\rightarrow \mathrm{HO}_{2}+\mathrm{HCHO}+\mathrm{OH}$ \\
\hline 10 & $\mathrm{~N}_{2} \mathrm{O}_{5}+h v$ & $\rightarrow \mathrm{NO}_{3}+\mathrm{NO}_{2}$ \\
\hline 11 & $\mathrm{NO}_{2}+h v$ & $\rightarrow \mathrm{NO}+\mathrm{O}\left({ }^{3} \mathrm{P}\right)$ \\
\hline $12 \mathrm{a}$ & $\mathrm{NO}_{3}+h v$ & $\rightarrow \mathrm{NO}+\mathrm{O}_{2}$ \\
\hline $12 b$ & $\mathrm{NO}_{3}+h v$ & $\rightarrow \mathrm{NO}_{2}+\mathrm{O}\left({ }^{3} \mathrm{P}\right)$ \\
\hline 14 & $\mathrm{O}_{2}+h v$ & $\rightarrow \mathrm{O}\left({ }^{3} \mathrm{P}\right)+\mathrm{O}\left({ }^{3} \mathrm{P}\right)$ \\
\hline $15 \mathrm{a}$ & $\mathrm{O}_{3}+h v$ & $\rightarrow \mathrm{O}_{2}+\mathrm{O}\left({ }^{1} \mathrm{D}\right)$ \\
\hline $15 b$ & $\mathrm{O}_{3}+h v$ & $\rightarrow \mathrm{O}_{2}+\mathrm{O}\left({ }^{3} \mathrm{P}\right)$ \\
\hline 17 & $\mathrm{PAN}+h v$ & $\rightarrow \mathrm{MeCO}_{3}+\mathrm{NO}_{2}$ \\
\hline 18 & $\mathrm{HONO}+h v$ & $\rightarrow \mathrm{OH}+\mathrm{NO}$ \\
\hline 19 & $\mathrm{EtCHO}+h v$ & $\rightarrow \mathrm{EtOO}+\mathrm{HO}_{2}+\mathrm{CO}$ \\
\hline 20 & $\mathrm{Me}_{2} \mathrm{CO}+h v$ & $\rightarrow \mathrm{MeCO}_{3}+\mathrm{MeOO}$ \\
\hline 21 & $\mathrm{n}-\mathrm{PrOOH}+h v$ & $\rightarrow \mathrm{EtCHO}+\mathrm{HO}_{2}+\mathrm{OH}$ \\
\hline 22 & $\mathrm{i}-\mathrm{PrOOH}+h v$ & $\rightarrow \mathrm{Me}_{2} \mathrm{CO}+\mathrm{HO}_{2}+\mathrm{OH}$ \\
\hline 23 & $\mathrm{MeCOCH}_{2} \mathrm{OOH}+h v$ & $\rightarrow \mathrm{MeCO}_{3}+\mathrm{HCHO}+\mathrm{OH}$ \\
\hline 24 & $\mathrm{PPAN}+h v$ & $\rightarrow \mathrm{EtCO}_{3}+\mathrm{NO}_{2}$ \\
\hline 25 & $\mathrm{MeONO}_{2}+h v$ & $\rightarrow \mathrm{HO}_{2}+\mathrm{HCHO}+\mathrm{NO}_{2}$ \\
\hline $26 \mathrm{a}$ & TERPOOH $+h v$ & $\rightarrow \mathrm{OH}+\mathrm{HO}_{2}+\mathrm{MACR}+\mathrm{MACR}$ \\
\hline $26 b$ & $\mathrm{TERPOOH}+h v$ & $\rightarrow \mathrm{TERPOOH}+\mathrm{Me}_{2} \mathrm{CO}$ \\
\hline 28 & $\mathrm{ISOOH}+h v$ & $\rightarrow \mathrm{OH}+\mathrm{MACR}+\mathrm{HCHO}+\mathrm{HO}_{2}$ \\
\hline 29 & ISON $+h v$ & $\rightarrow \mathrm{NO}_{2}+\mathrm{MACR}+\mathrm{HCHO}+\mathrm{HO}_{2}$ \\
\hline 30 & $\mathrm{MACR}+h v$ & $\rightarrow \mathrm{MeCO}_{3}+\mathrm{HCHO}+\mathrm{CO}+\mathrm{HO}_{2}$ \\
\hline 31 & $\mathrm{MPAN}+h v$ & $\rightarrow \mathrm{MACRO}_{2}+\mathrm{NO}_{2}$ \\
\hline $32 \mathrm{a}$ & $\mathrm{MACROOH}+h v$ & $\rightarrow \mathrm{OH}+\mathrm{HO}_{2}+\mathrm{OH}+\mathrm{HO}_{2}$ \\
\hline $32 b$ & $\mathrm{MACROOH}+h v$ & $\rightarrow \mathrm{HACET}+\mathrm{CO}+\mathrm{MGLY}+\mathrm{HCHO}$ \\
\hline 34 & $\mathrm{HACET}+h v$ & $\rightarrow \mathrm{MeCO}_{3}+\mathrm{HCHO}+\mathrm{HO}_{2}$ \\
\hline 35 & MGLY + hv & $\rightarrow \mathrm{MeCO}_{3}+\mathrm{CO}+\mathrm{HO}_{2}+$ \\
\hline 36 & $\mathrm{NALD}+h v$ & $\rightarrow \mathrm{HCHO}+\mathrm{CO}+\mathrm{NO}_{2}+\mathrm{HO}_{2}$ \\
\hline 37 & $\mathrm{MeCO}_{3} \mathrm{H}+h v$ & $\rightarrow \mathrm{MeOO}+\mathrm{OH}$ \\
\hline $38 \mathrm{a}$ & $\mathrm{O}_{3} \mathrm{~S}+h v$ & $\rightarrow \mathrm{O}_{2}+\mathrm{O}(1 \mathrm{D}) \mathrm{S}$ \\
\hline $38 b$ & $\mathrm{O}_{3} \mathrm{~S}+h v$ & $\rightarrow \mathrm{O}_{2}+\mathrm{O}(3 \mathrm{P}) \mathrm{S}$ \\
\hline $40 \mathrm{a}$ & $\mathrm{BtOOH}+h v$ & $\rightarrow \mathrm{MEK}+\mathrm{MEK}+\mathrm{EtOO}+\mathrm{MeCHO}$ \\
\hline $40 \mathrm{~b}$ & $\mathrm{BtOOH}+h v$ & $\rightarrow \mathrm{HO}_{2}+\mathrm{HO}_{2}$ \\
\hline $40 \mathrm{c}$ & $\mathrm{BtOOH}+h v$ & $\rightarrow \mathrm{OH}+\mathrm{OH}+\mathrm{OH}$ \\
\hline 43 & $\mathrm{MEK}+h v$ & $\rightarrow \mathrm{MeCO}_{3}+\mathrm{EtOO}$ \\
\hline 44 & $\mathrm{MeCOCOMe}+h v$ & $\rightarrow \mathrm{MeCO}_{3}+\mathrm{MeCO}_{3}$ \\
\hline 45 & $\mathrm{MEKOOH}+h v$ & $\rightarrow \mathrm{MeCO}_{3}+\mathrm{MeCHO}+\mathrm{OH}$ \\
\hline $46 \mathrm{a}$ & $\mathrm{ONIT}+h v$ & $\rightarrow \mathrm{NO}_{2}+\mathrm{MEK}+\mathrm{HO}_{2}+\mathrm{EtOO}$ \\
\hline $46 b$ & $\mathrm{ONIT}+h v$ & $\rightarrow \mathrm{MeCHO}+\mathrm{ONIT}$ \\
\hline $48 \mathrm{a}$ & $\mathrm{AROMOOH}+h v$ & $\rightarrow \mathrm{OH}+\mathrm{Me}_{2} \mathrm{CO}+\mathrm{HO}_{2}+\mathrm{CO}$ \\
\hline $48 b$ & $\mathrm{AROMOOH}+h v$ & $\rightarrow \mathrm{MeCO}_{3}+\mathrm{AROMOOH}$ \\
\hline
\end{tabular}

Breider et al., 2010). When TOMCAT is run uncoupled to GLOMAP, there is an option to account for heterogeneous uptake of $\mathrm{N}_{2} \mathrm{O}_{5}$ using prescribed monthly mean aerosol number density and radius for five different aerosol types (sulfate, black carbon, organic carbon, sea salt and dust). Currently, these data are taken from a previous GLOMAP run for the year 2000 (Mann et al., 2010). In this simplified scheme, the uptake coefficients are based on Evans and Jacob (2005), 
Table 6. List of $\gamma$ values used in TOMCAT for heterogeneous uptake of $\mathrm{N}_{2} \mathrm{O}_{5}$ by aerosol.

\begin{tabular}{ll}
\hline Aerosol type & $\begin{array}{l}\text { Reaction probability } \\
(T=\text { temperature }(\mathrm{K}), \\
\end{array}$ \\
& $\mathrm{RH}=$ relative humidity $(\%))$ \\
\hline Sulfate & $\gamma=\alpha \times 10^{\beta}$ \\
& $\alpha=2.79 \times 10^{-4}+1.3$ \\
& $\times 10^{-4} \times \mathrm{RH}-3.43$ \\
& $\times 10^{-6} \times \mathrm{RH}^{2}+7.52$ \\
& $\times 10^{-8} \times \mathrm{RH}^{3}$ \\
& $\beta=4 \times 10^{-2} \times(T-294)(T \geq 282 \mathrm{~K})$ \\
& $\beta=-0.48(T<282 \mathrm{~K})$ \\
Organic Carbon & $\gamma=\mathrm{RH} \times 5.2 \times 10^{-4}(\mathrm{RH}<57 \%)$ \\
& \\
Black Carbon & $\gamma=0.005$ \\
Sea Salt & $\gamma=0.005(\mathrm{RH}<62 \%)$ \\
& $\gamma=0.03(\mathrm{RH} \geq 62 \%)$ \\
Dust & $\gamma=0.02$ \\
\hline
\end{tabular}

with the exception of dust, which is based on Mogili et al. (2006) (see Table 6). The overall uptake coefficient varies as a function of temperature, humidity and aerosol composition. Similarly, computationally cheap TOMCAT-GLOMAP "aerosol-only" experiments can be run using specified fields of oxidants. Uptake of $\mathrm{N}_{2} \mathrm{O}_{5}$ on cloud surfaces is currently not included due to the use of climatological clouds in the model. Code exists to take account of $\mathrm{HO}_{2}$ uptake but is currently not used in the model as it requires evaluation and testing within the model.

\subsection{Model set-up and emissions}

A simulation has been performed using the current chemical mechanism scheme for the year 2008 (with a 1-year spinup). The model uses 31 vertical levels (surface to $10 \mathrm{hPa}$ ) and a horizontal resolution of $2.8^{\circ} \times 2.8^{\circ}$. ERA-Interim meteorology is used to drive the model. Offline aerosol concentrations are used for $\mathrm{N}_{2} \mathrm{O}_{5}$ uptake. This run uses emissions that were chosen for the POLARCAT (POLar study using Aircraft, Remote Sensing, surface measurements and models of Climate, chemistry, Aerosols, and Transport) Model Intercomparison Project (POLMIP) (Emmons et al., 2015). Monthly mean anthropogenic and ship emissions are based on the Streets v1.2 inventory, which was updated with several recent regional inventories in 2008 for the POLARCAT campaign (see Table 1). This included Asian emissions from Zhang et al. (2009), North American emissions from USNEI 2002 and CAC 2005, and European emissions from the EMEP 2006 database (http://www.ceip.at). Where regional inventories were unavailable, emissions were taken from the
EDGAR 3.2FT2000 database. These emissions have no seasonal cycle and are therefore the same each month. Monthly varying biogenic emissions are from the MACC (Monitoring Atmospheric Composition and Climate) project (MACCity), which provides simulated VOCs calculated offline by the Model of Emissions of Gases and Aerosols from Nature (MEGAN) v2.0 for the reference year 2000 (Guenther et al., 2006). Oceanic CO and VOC emissions and soil $\mathrm{NO}_{x}$ are from the POET inventory. For 2008, daily biomass burning emissions are taken from the Fire INventory from NCAR (FINN) (Wiedinmyer et al., 2011). Aircraft emissions of $\mathrm{NO}_{x}$ are based on estimated aircraft movements for the year 2002 (Lamarque et al., 2010) and were calculated for the European QUANTIFY project (http://www.pa.op.dlr.de/ quantify/). They are provided on 25 vertical levels from the surface to $14.5 \mathrm{~km}$ and are regridded to the TOMCAT vertical levels online. Surface $\mathrm{CH}_{4}$ is set to equal zonal mean concentrations calculated from NOAA/ESRL/GMD surface observations for the year 2000 (Meinshausen et al., 2011). There is also the option to use an emission file for $\mathrm{CH}_{4}$ and then scale the surface global mean concentration to a suitable value for the year in question. As already mentioned, lightning $\mathrm{NO}_{x}$ emissions are also included but are dealt with online depending on the convection in the model.

\section{Observations}

\subsection{Satellite data}

Simulated CO is compared on a global scale to $\mathrm{CO}$ distributions retrieved from the satellite instrument, MOPITT (Measurements Of Pollution In The Troposphere) version 6. MOPITT is a nadir-viewing instrument onboard the NASA Terra satellite and retrieves $\mathrm{CO}$ concentrations globally at a horizontal resolution of $\sim 22 \mathrm{~km}$ by measuring infrared radiances in the CO absorption band (Deeter et al., 2010). The Terra satellite has an overpass time at the Equator of 10:30 local time (LT). Version 6 uses an a priori based on climatological output from the CAM-Chem model for 2000 to 2009 (Deeter, 2013). It has increased sensitivity to lower tropospheric CO by using both near-infrared and thermal infrared wavelengths (Deeter et al., 2011). As MOPITT is a nadir-viewing instrument, it is more sensitive to certain altitudes; therefore, averaging kernels (AKs) that contain information about the instrument's varying sensitivities at different altitudes are used, along with the a priori, to apply the same vertical sensitivity to the TOMCAT CO profiles. This allows a more accurate comparison between the observed and simulated CO. Incidents where the degrees of freedom signal (DOFS) is less than 1 are used to identify data where the satellite sensitivity is low. These data points have been removed from both the satellite and model columns.

Satellite $\mathrm{O}_{3}$ is taken from Global Ozone Monitoring Experiment-2 (GOME-2) aboard EUMETSAT's Metop-A 
Table 7. Model diagnostics compared to previously published values.

\begin{tabular}{llll}
\hline Diagnostic & TOMCAT & Published values & Reference \\
\hline $\mathrm{O}_{3}$ Burden $(\mathrm{Tg})^{\mathrm{a}}$ & 331 & $337 \pm 23$ & Young et al. (2013) \\
$\left.\begin{array}{l}\mathrm{OH} \text { concentration } \\
\left(\times 10^{6} \text { molecules cm }\right.\end{array}{ }^{-3}\right)^{\mathrm{b}}$ & 1.08 & $0.94-1.06$ & $\begin{array}{l}\text { Krol and Lelieveld (2003); Prinn et al. (2001), } \\
\mathrm{CH}_{4} \text { lifetime (yrs) }\end{array}$ \\
\hline
\end{tabular}

${ }^{\mathrm{a}}$ Annual mean. ${ }^{\mathrm{b}}$ Mass-weighted annual mean.

(a) CO measurements

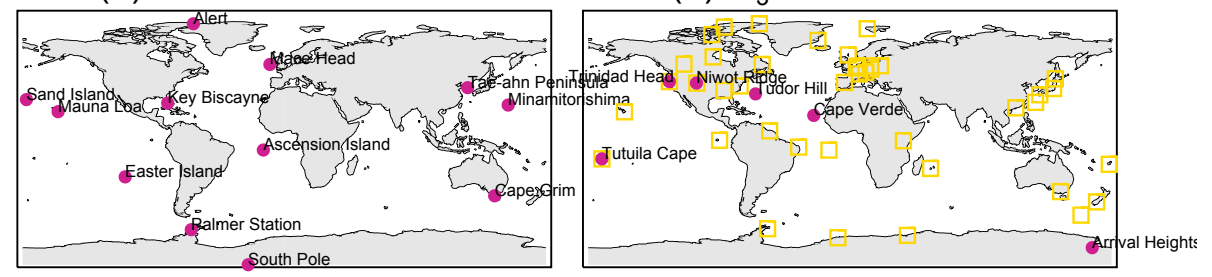

(c) $\mathrm{NO}_{x}$, PAN and

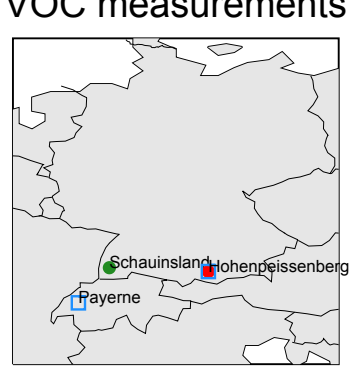

(b) $\mathrm{O}_{3}$ measurements

\begin{tabular}{l} 
WDCGG CO and $\mathrm{O}_{3}$ \\
$\mathrm{O}_{3}$ sonde data \\
WDCGG VOCs \\
WDCGG PAN \\
WDCGG NO \\
\hline
\end{tabular}

Figure 2. Location of the World Data Centre for Greenhouse Gases (WDCGG) surface observatories and ozonesonde release sites used to evaluate the model for (a) $\mathrm{CO}$, (b) $\mathrm{O}_{3}$ and (c) PAN, $\mathrm{NO}_{x}$ and VOCs.

polar-orbiting satellite. GOME-2 is a nadir-viewing instrument with an approximate local Equator crossing time of 09:30 LT. It has a spectral range of $240-790 \mathrm{~nm}$ and the pixel sizes are between 40 and $80 \mathrm{~km}$ along and across track, respectively (Miles et al., 2015b). The data come from the Rutherford Appleton Laboratory and are based on an optimal estimation algorithm (Rodgers, 1976). Miles et al. (2015b) describes how the GOME-2 retrievals are quality controlled prior to use, with data being removed where geometric cloud fraction is greater than 0.2 and the solar zenith angle is less than $80^{\circ}$. For optimal comparisons, the GOME-2 AKs are applied to the TOMCAT data, as described in Miles et al. (2015a). The model and satellite data are matched in space and time by choosing the closest model grid box to the satellite pixel, to within $3 \mathrm{~h}$ of the satellite daylight overpass time (6-hourly model output is being used).

For nitrogen dioxide $\left(\mathrm{NO}_{2}\right)$, we use data from the Ozone Monitoring Instrument (OMI) aboard NASA's EOS-Aura polar-orbiting satellite. It has an approximate Equator crossing of 13:30 LT (Boersma et al., 2007) and is a nadirviewing instrument with a spectral range of $270-500 \mathrm{~nm}$. The pixel sizes are between $16-23 \mathrm{~km}$ and $24-135 \mathrm{~km}$ along and across track, respectively, depending on the viewing zenith angle (Boersma et al., 2007). The tropospheric column $\mathrm{NO}_{2}$ data, known as the DOMINO product (v2.0) (Boersma et al., 2011), were downloaded from the Tropospheric Emissions Monitoring Internet Service (TEMIS; http://www.temis.nl/airpollution/no2.html). The retrieval of OMI tropospheric column $\mathrm{NO}_{2}$ is based on differential optical absorption spectroscopy (DOAS), as discussed by Eskes and Boersma (2003). OMI retrievals have been quality controlled, and data are only used where they have geometric cloud cover less than $20 \%$ and good-quality data flags. The product also uses the algorithm of Braak (2010) to remove OMI pixels affected by row anomalies. Studies have shown the DOMINO product to have small biases against other independent observational data with some evidence of a small low bias over oceans (Irie et al., 2012; Boersma et al., 2008). The product has also been used in model evaluation studies previously (e.g. Huijnen et al., 2010; Pope et al., 2015). For the TOMCAT comparisons, AKs are applied following Boersma et al. (2011). The model and satellite data are matched in space and time by choosing the closest model 
(a) $\mathrm{CO} 2008$
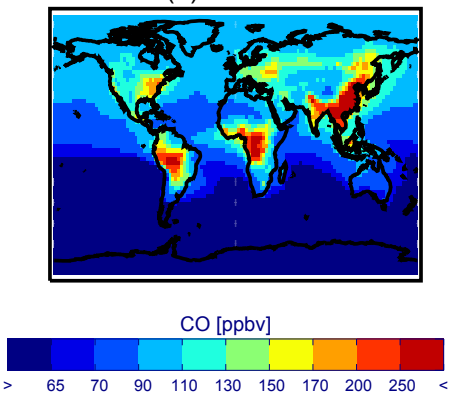

(b) CO 2008

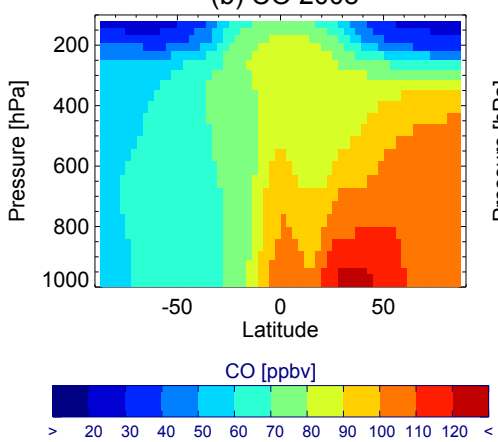

(c) $\mathrm{O}_{3} 2008$

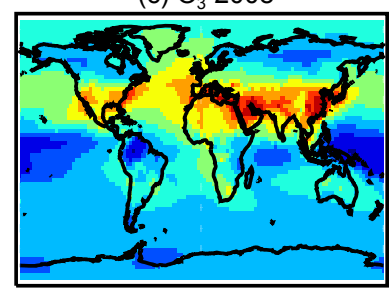

$\mathrm{O}_{3}[\mathrm{ppbv}]$

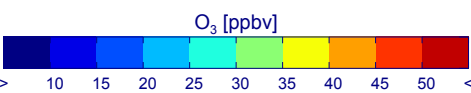

(e) $\mathrm{OH} 2008$
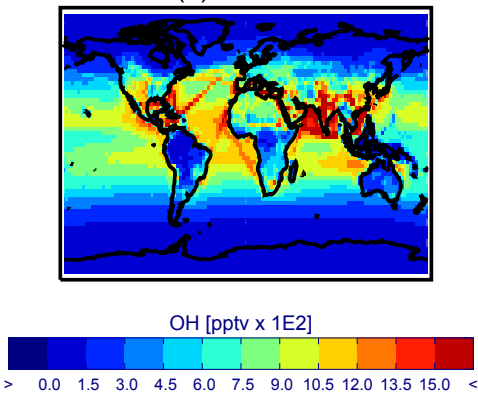

(f) $\mathrm{OH} 2008$
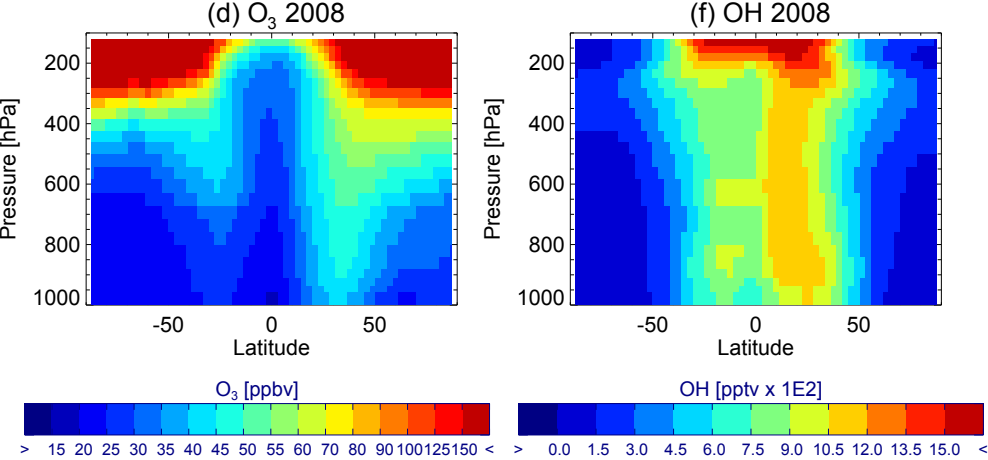

Figure 3. Concentrations of TOMCAT-simulated annual surface mean and annual zonal mean $\mathrm{CO}(\mathbf{a}, \mathbf{b}), \mathrm{O}_{3}(\mathbf{c}, \mathbf{d})$ and $\mathrm{OH}(\mathbf{e}, \mathbf{f})$.

grid box to the satellite pixel, to within $3 \mathrm{~h}$ of the satellite daylight overpass time (6-hourly model output is being used).

\subsection{Surface data}

We take $\mathrm{O}_{3}$ measurements at the surface over the US from the United States Environmental Protection Agency (EPA) Clean Air Status and Trends Network (CASTNET) database. They provide hourly mean concentrations from continuous ozone monitoring instruments that have undergone a large amount of quality assurance. Here, we use data from 44 sites, which cover large parts of the US, excluding highly urbanised sites as identified by Sofen et al. (2016). The model output is interpolated to the location of each station both horizontally and vertically.

Observations of CO, VOCs, peroxyacetyl nitrate (PAN) and some $\mathrm{O}_{3}$ measurements are taken from the World Data Centre for Greenhouse Gases (WDCGG; http://ds.data.jma. go.jp/gmd/wdcgg/; see Fig. 2 for locations). Most of the surface $\mathrm{O}_{3}$ and $\mathrm{CO}$ measurements are provided by the National Oceanic and Atmospheric Administration (NOAA). NOAA $\mathrm{CO}$ is from flask samples that have been analysed using gas chromatography (Novelli et al., 1998) and $\mathrm{O}_{3}$ is measured by ultraviolet (UV) light absorption at $254 \mathrm{~nm}$ (Oltmans and Levy, 1994). The $\mathrm{O}_{3}$ measurements at Cabo Verde are provided by the University of York and were made using a UV light absorption instrument (Read et al., 2008). CO at Minami Torishima is from continuous measurements made by the Japan Meteorological Agency (JMA) using gas chro- matography (Watanabe et al., 2000). PAN measurements at Zugspitze and Schauinsland are provided to the WDCGG by the German Federal Environment Agency (UBA) and were made using a commercial gas chromatograph (GC) analyser (Pandey Deolal et al., 2014). VOC measurements of ethene, ethane, propene, propane, toluene and butane made using gas chromatography at Hohenpeissenberg were provided by the German Meteorological Service (DWD) (PlassDülmer et al., 2002). All NO $\mathrm{N}_{x}$ measurements were made using chemiluminescence and are provided by DWD at Hohenpeissenberg (Mannschreck et al., 2004), UBA at Zugspitze, Empa (Swiss Federal Laboratories for Materials Science and Technology) at Jungfraujoch, Payerne and Rigi (Zellweger et al., 2003), and by RIVM (Netherlands National Institute for Public Health and the Environment) at Kollumerwaard.

\subsection{Ozonesonde data climatology}

Simulated $\mathrm{O}_{3}$ profiles are compared to ozonesonde data from a climatology, which uses 17 years of ozone balloon soundings made between 1995 and 2011 (Tilmes et al., 2012). The data are available as profiles between 1000 and $10 \mathrm{hPa}$ at 42 stations, covering large parts of the globe. The model output is interpolated to the longitude and latitude of each station location. The site locations are shown in Fig. 2b. The ozonesondes tend to measure concentrations around $10 \mathrm{ppbv}$ higher over the eastern US and around 5 ppbv lower over Europe compared to independent observational data from aircraft and surface data (Tilmes et al., 2012). For comparison 
to TOMCAT, both the model and the observations have been averaged into three different altitude and latitude bands for comparison.

\subsection{Aircraft ARCTAS data}

We use observations taken aboard the DC- 8 aircraft between 29 June and 10 July 2008 during the Arctic Research of the Composition of the Troposphere from Aircraft and Satellites (ARCTAS) campaign (Jacob et al., 2010). At this time, the aircraft was based at Cold Lake, Canada, and flew over large parts of North America and the Arctic. For the comparisons, the model monthly mean data for July are used and are averaged over a region contained by the maximum and minimum longitude and latitude ranges of the aircraft during the campaign.

\subsection{OH estimates}

$\mathrm{OH}$ is difficult to measure due to its very short lifetime ( $1 \mathrm{~s})$ and low concentrations, and even though vast improvements have been made to in situ measurement techniques (Heard and Pilling, 2003), they do not provide a global picture. A common method to estimate $\mathrm{OH}$ is by using measurements of methyl chloroform $\left(\mathrm{CH}_{3} \mathrm{CCl}_{3}, \mathrm{MCF}\right)$, for which the primary loss channel is through reaction with $\mathrm{OH}$. Accurate determination of $\mathrm{OH}$ from MCF relies on accurate estimation of emissions and the use of models, introducing possible biases. These measurements are frequently used to estimate the global mean $\mathrm{OH}$ concentration (e.g. Krol et al., 1998; Prinn et al., 2001; Montzka et al., 2011) and can offer some insight into the regional distribution of $\mathrm{OH}$ (e.g. Krol and Lelieveld, 2003; Patra et al., 2014). We use published estimates of global mean $\mathrm{OH}$ to discuss possible biases in simulated $\mathrm{OH}$ in Sect. 4.2.

\section{Results}

\subsection{Simulated distributions of $\mathrm{CO}, \mathrm{O}_{3}$ and $\mathrm{OH}$}

Figure 3 shows annual mean surface and zonal mean concentrations of $\mathrm{CO}, \mathrm{O}_{3}$ and $\mathrm{OH}$ from the TOMCAT 2008 simulation. CO is emitted directly from natural and anthropogenic sources and produced in the atmosphere from chemical destruction of VOCs (Logan et al., 1981). Direct emission at the Earth's surface and secondary production in the troposphere from VOCs (most notably $\mathrm{CH}_{4}$ ) are estimated to be of equal importance in terms of total global tropospheric $\mathrm{CO}$ sources (Duncan et al., 2007). High concentrations due to direct emission of $\mathrm{CO}$ from fossil fuel burning can be seen in Fig. 3a in the densely populated regions of North America, central Europe and Asia. Large concentrations are also seen over regions with high rates of biomass burning, such as South America and Africa. Both at the surface and throughout the troposphere, higher background concentrations of $\mathrm{CO}$ (a) Spivakovsky dataset
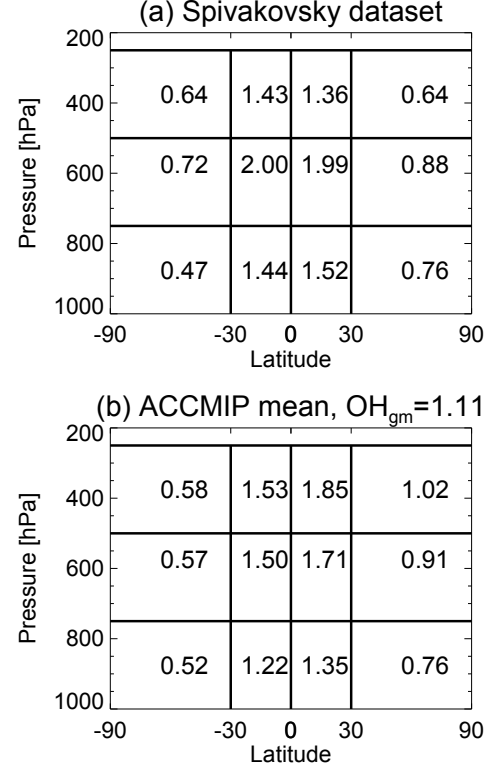

(c) TOMCAT, $\mathrm{OH}_{\mathrm{gm}}=1.08$

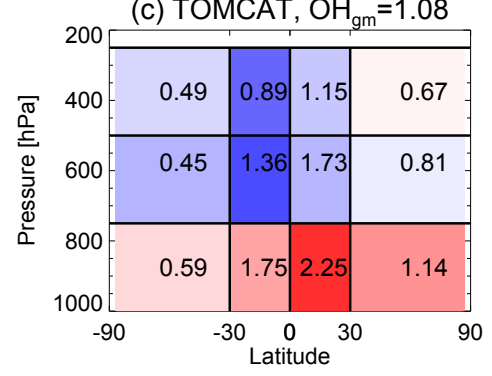

Figure 4. Regional annual mean $\mathrm{OH}$ concentrations $\left(\times 10^{6}\right.$ molecules $\left.\mathrm{cm}^{-3}\right)$ split into subsections as recommended by Lawrence et al. (2001). (a) OH estimated from methyl chloroform observations from Spivakovsky et al. (2000), (b) the Atmospheric Chemistry and Climate Model Intercomparison Project (ACCMIP) multi-model mean simulated $\mathrm{OH}$ concentrations from Naik et al. (2013) and (c) TOMCAT-simulated OH concentrations for the year 2008. The air-mass-weighted global mean tropospheric $\mathrm{OH}\left(\mathrm{OH}_{\mathrm{gm}}\right)$ is indicated above each plot for panels (b) and (c). In TOMCAT, the troposphere was defined as the area below a climatological tropopause $\left(p=300-215(\cos (\text { lat }))^{2}\right)$ (as discussed in Lawrence et al. (2001)) and for ACCMIP it was defined as below $200 \mathrm{hPa}$. The colours in panel (c) are scaled according to the difference from panel (a), with the darkest blue representing the largest negative differences and the darkest red representing the largest positive differences.

are seen in the Northern Hemisphere $(\mathrm{NH})$ due to larger emissions.

$\mathrm{O}_{3}$ is important in the troposphere as it is a major source of $\mathrm{OH}$, the primary oxidising agent in the troposphere, and is an air pollutant and greenhouse gas (Monks et al., 2015a). It is not directly emitted but produced from photochemical reactions involving $\mathrm{NO}_{x}$, VOCs and $\mathrm{CO}$, and is transported from the stratosphere to the troposphere (Lelieveld and Dentener, 2000). The atmospheric burden of $\mathrm{O}_{3}$ is controlled by 
(a) MOPITT April 2008
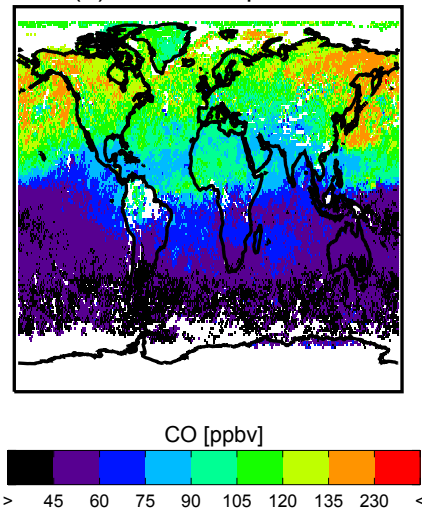

(d) MOPITT October 2008

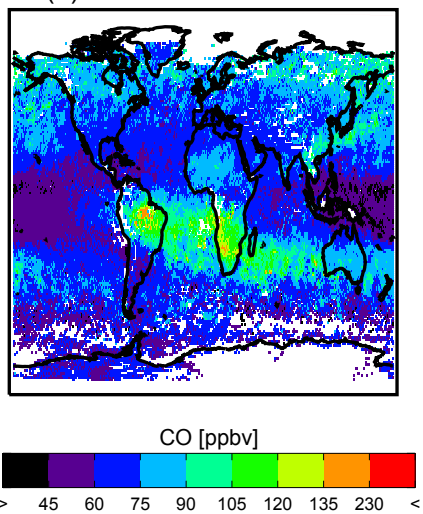

(b) TOMCAT April 2008

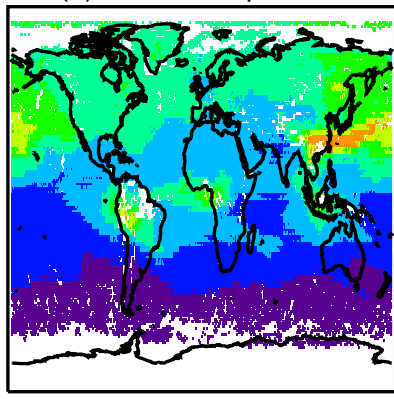

$\mathrm{CO}[\mathrm{ppbv}]$

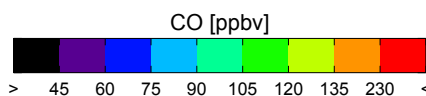

(e) TOMCAT October 2008

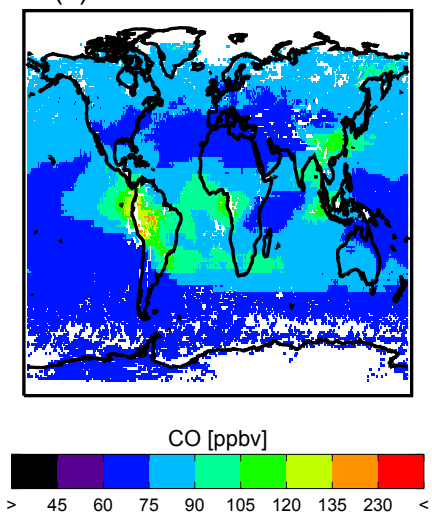

(c) April 2008

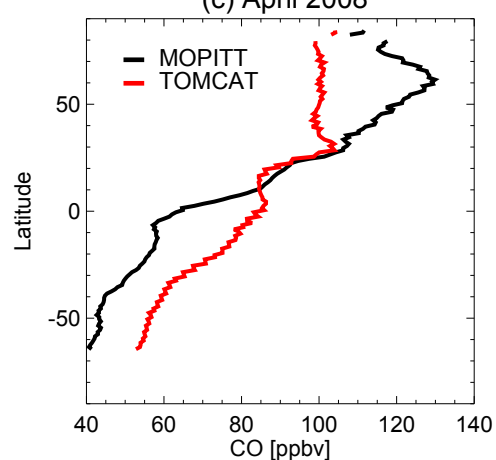

(f) October 2008

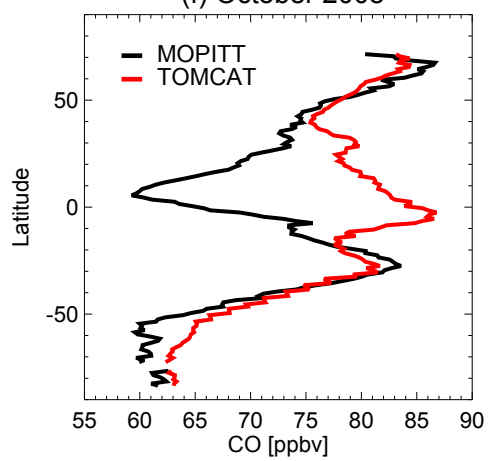

Figure 5. April and October 2008 monthly mean $500 \mathrm{hPa}$ CO concentrations (ppbv) observed by MOPITT (a, d) and simulated by TOMCAT $(\mathbf{b}, \mathbf{e})$. The zonal mean concentrations at $500 \mathrm{hPa}$ are also shown $(\mathbf{c}, \mathbf{f}$; data are only shown when there is $>25 \%$ coverage in a given latitude band). MOPITT averaging kernels have been applied to the TOMCAT fields.

a balance between these sources and loss through chemical reactions and deposition (Stevenson et al., 2006). Figure $3 \mathrm{c}$ shows the highest concentrations at the surface lie within the $\mathrm{NH}$ extratropical region due to the proximity to large emissions of $\mathrm{NO}_{x}$ and VOCs, and photochemical production. Some of the highest concentrations of $\mathrm{O}_{3}$ are found downwind of regions with high $\mathrm{NH}$ anthropogenic emissions (identified by $\mathrm{CO}$ in Fig. 3a). This is due to production of $\mathrm{O}_{3}$ being greater downwind of source regions away from very high $\mathrm{NO}_{x}$ concentrations that can titrate $\mathrm{O}_{3}$ in urban environments (Monks et al., 2015a). Low $\mathrm{O}_{3}$ over the central Pacific Ocean and northern South America is also seen in the model. In the tropics, lower $\mathrm{O}_{3}$ concentrations are seen at $100-300 \mathrm{hPa}$ due to a higher tropopause in this region and the uplift of air with low $\mathrm{O}_{3}$ within deep tropical convection. At around $20-40^{\circ} \mathrm{S} / \mathrm{N}$, evidence of the downward transport of stratospheric $\mathrm{O}_{3}$ by the Brewer-Dobson circulation (Butchart, 2014) can be seen. The overall features of TOMCAT $\mathrm{O}_{3}$ are consistent with multi-model results from the Atmospheric Chemistry and Climate Model Intercomparison Project (ACCMIP) (Young et al., 2013) and those observed by satellite (Ziemke et al., 2011). TOMCAT sim- ulates an annual mean tropospheric burden of $331 \mathrm{Tg}$ (see Table 7), which agrees well with the present-day ACCMIP multi-model mean tropospheric ozone burden of $337 \pm 23 \mathrm{Tg}$ (Young et al., 2013).

$\mathrm{OH}$ is the dominant radical responsible for the removal of pollutants such as $\mathrm{NO}_{x}$ and VOCs from the atmosphere, initiating the production of $\mathrm{O}_{3}$ (Gligorovski et al., 2015) and aerosols (e.g. Carlton et al., 2009). OH is produced in the troposphere when $\mathrm{O}_{3}$ is photolysed to produce $\mathrm{O}\left({ }^{1} \mathrm{D}\right)$ and a subsequent reaction with $\mathrm{H}_{2} \mathrm{O}$. It is therefore produced in large quantities in the tropics, where there are large concentrations of $\mathrm{H}_{2} \mathrm{O}$ and a high incidence of solar radiation. This can be seen in TOMCAT in Fig. 3e and $\mathrm{f}$ with high concentrations of $\mathrm{OH}$ occurring between $50^{\circ} \mathrm{N}$ and $50^{\circ} \mathrm{S}$. The spatial distribution of TOMCAT surface $\mathrm{OH}$ is broadly similar to multi-model surface $\mathrm{OH}$ from the ACCMIP study shown by Voulgarakis et al. (2013).

\subsection{Evaluation of $\mathrm{OH}$}

TOMCAT global mean air-mass-weighted tropospheric $\mathrm{OH}$ was calculated using a climatological tropopause (see definition in Fig. 4) following Lawrence et al. (2001). TOM- 

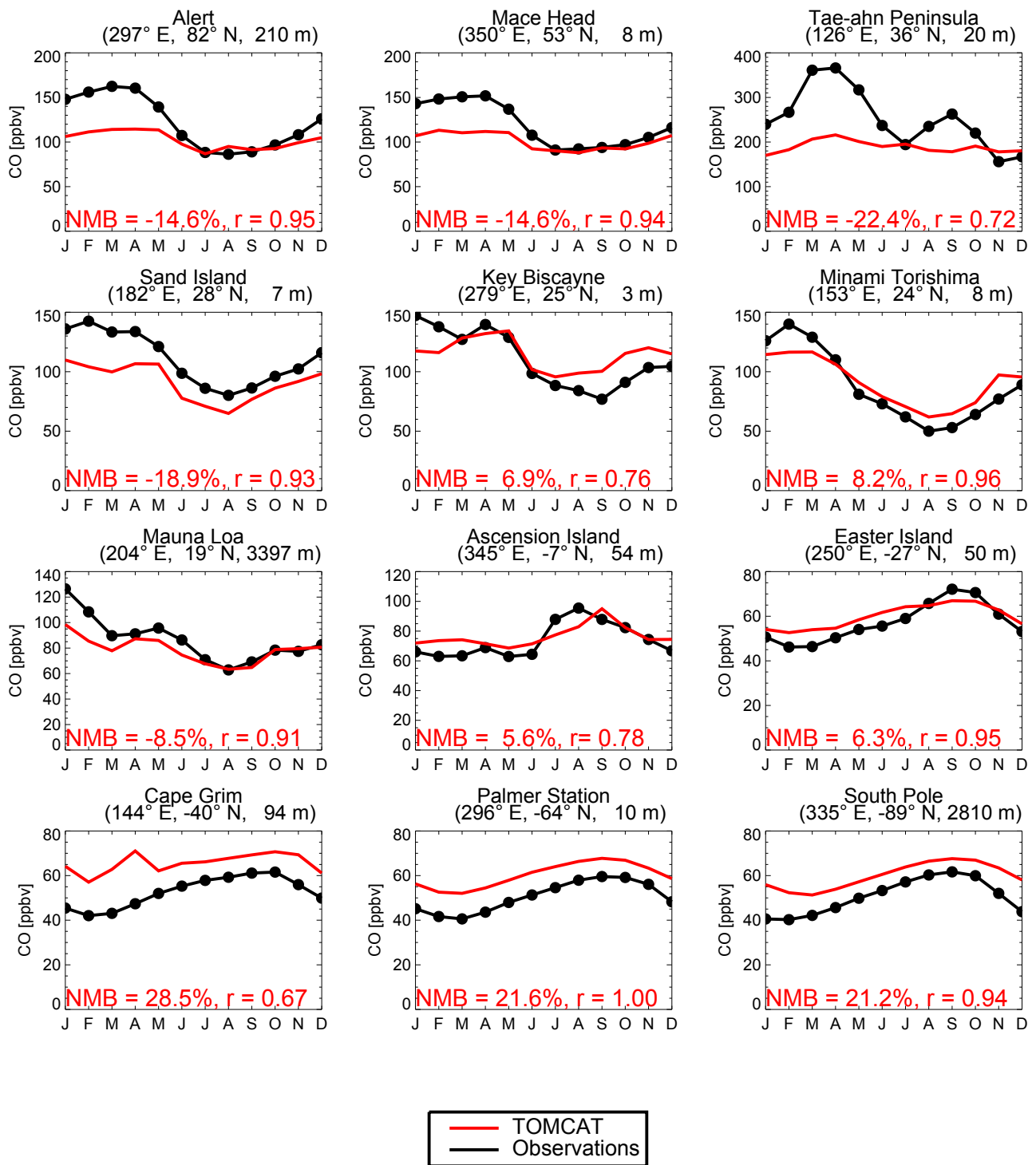

Figure 6. Monthly mean 2008 observed and simulated CO (ppbv) at several surface sites located throughout the globe. The panels are arranged by latitude from north to south, with Pearson correlation $(r)$ and normalised mean bias (NMB) between the observed and simulated monthly mean data printed on each panel.

CAT has an annual mean tropospheric $\mathrm{OH}$ concentration of $1.08 \times 10^{6}$ molecules $\mathrm{cm}^{-3}$. Concentrations of global mean tropospheric $\mathrm{OH}$ calculated from $\mathrm{MCF}$ observations have been estimated to be $0.94 \times 10^{6}$ molecules $\mathrm{cm}^{-3}$ by Prinn et al. (2001), $1.0 \times 10^{6}$ molecules $\mathrm{cm}^{-3}$ by Krol et al. (2003) and $0.98 \times 10^{6}$ molecules $\mathrm{cm}^{-3}$ by Bousquet et al. (2005). These estimates indicate that the TOMCAT global mean $\mathrm{OH}$ may be slightly high. However, a recent inverse modelling study calculated a global mean $\mathrm{OH}$ concentration of $1.06 \times 10^{6}$ molecules $\mathrm{cm}^{-3}$, highlighting uncertainties in using MCF observations to calculate $\mathrm{OH}$ (Wang et al., 2008). In addition to this, concentrations reported by model intercomparison studies are also higher than those reported based on observations. The POLARCAT Model
Intercomparison Project (POLMIP) found a multi-model mean value of $1.08 \pm 0.6 \times 10^{6}$ molecules $\mathrm{cm}^{-3}$ when using eight models (including a previous version of TOMCAT). The multi-model mean was the same whether a climatological tropopause was used, as done here, or when the $150 \mathrm{ppb} \mathrm{O}_{3}$ contour line was used. Voulgarakis et al. (2013) found a multi-model mean concentration of $1.17 \pm 0.1 \times$ $10^{6}$ molecules $\mathrm{cm}^{-3}$ when using a subset of 12 ACCMIP models, and Naik et al. (2013) found a multi-model mean of $1.11 \pm 0.2 \times 10^{6}$ molecules $\mathrm{cm}^{-3}$ when using all 16 ACCMIP models. Both of these ACCMIP concentrations were calculated using a tropopause of $200 \mathrm{hPa}$. However, Voulgarakis et al. (2013) found little difference in the resulting concentrations of $\mathrm{OH}$ when using different methods of defining the 
tropopause $\left(200 \mathrm{hPa}, 150 \mathrm{ppbv} \mathrm{O}_{3}\right.$ contour and the climatological tropopause, as used here).

Whilst comparing the global mean $\mathrm{OH}$ concentration in TOMCAT to those reported in the literature is very useful, it is also important to consider the regional distribution of $\mathrm{OH}$ in TOMCAT. Figure 4 shows TOMCAT OH averaged into nine regional subsections defined by Lawrence et al. (2001), along with $\mathrm{OH}$ from Spivakovsky et al. (2000) (referred to as the Spivakovsky dataset) and the multi-model mean $\mathrm{OH}$ from the ACCMIP study (Naik et al., 2013). Patra et al. (2011) used the Spivakovsky dataset in a recent multi-model intercomparison project, but revised the concentrations down by $8 \%$ to match more recent measurements of MCF. This highlights that quantitative comparison of TOMCAT OH with the Spivakovsky dataset is limited due to observational and modelling uncertainties. However, the Spivakovsky dataset is still valuable for estimating the regional distribution of $\mathrm{OH}$.

The largest concentrations of $\mathrm{OH}$ are found in the tropics for the Spivakovsky dataset and for the ACCMIP and TOMCAT simulations. However, the ACCMIP models have the highest $\mathrm{OH}$ concentrations between 500 and $250 \mathrm{hPa}$, Spivakovsky has the highest concentrations between 750 and $500 \mathrm{hPa}$ and TOMCAT has the highest concentrations between the surface and $750 \mathrm{hPa}$. Large differences in the spatial distribution of simulated $\mathrm{OH}$ have recently been identified in models, highlighting uncertainties in the ability of current models to accurately simulate $\mathrm{OH}$ concentrations and distributions (Emmons et al., 2015; Monks et al., 2015b). TOMCAT was shown to have lower photolysis rates in the upper troposphere and higher photolysis rates in the lower troposphere compared to other models, with model differences in clouds and water vapour in the POLMIP models being identified as possible reasons for differences in the $\mathrm{OH}$ (Emmons et al., 2015; Monks et al., 2015b).

In addition to this, Patra et al. (2014) found that the NH to $\mathrm{SH}$ ratio of $\mathrm{OH}$, inferred from observations of $\mathrm{MCF}$, is equal to 0.97. TOMCAT has an annual $\mathrm{NH}$ : $\mathrm{SH}$ ratio of 1.37. Naik et al. (2013) found a $\mathrm{NH}: \mathrm{SH}$ ratio of $1.28 \pm 0.1$ for the ACCMIP models, which is also higher than that estimated from observations, indicating that this is a common feature in global models.

TOMCAT $\mathrm{OH}$ results in a chemical methane lifetime of 7.9 years. Voulgarakis et al. (2013) found an ACCMIP multimodel mean methane lifetime of $9.3 \pm 0.9$, with a minimum of 7.1 years and a maximum of 13.9 years. This indicates TOMCAT has a methane lifetime that is generally shorter than other models. As the majority of methane oxidation occurs in the tropics near the surface (Lawrence et al., 2001; Bloss et al., 2005), the short methane lifetime is likely due to TOMCAT having a higher concentration of $\mathrm{OH}$ in this region compared to other models.

\subsection{Evaluation of carbon monoxide}

As mentioned in Sect. 4.1, CO is emitted from a wide range of natural and anthropogenic sources and can provide insight into model emissions and subsequent transport of sources due to its lifetime of several months. Figure 5 shows retrieved $\mathrm{CO}$ from MOPITT (see Sect. 3) at $500 \mathrm{hPa}$ during April and October 2008 along with simulated CO from TOMCAT with the MOPITT averaging kernels applied.

In April, both the model and the satellite show higher $\mathrm{CO}$ concentrations in the NH compared to the Southern Hemisphere $(\mathrm{SH})$ due to a longer $\mathrm{CO}$ lifetime at this time of year in conjunction with higher anthropogenic emissions in the $\mathrm{NH}$. MOPITT observes concentrations around 10-30 ppbv larger than simulated in the NH midlatitudes and Arctic (Fig. 5c). This negative model bias is a well-known problem with current CTMs during winter and spring, with models having a 15 to $50 \mathrm{ppbv}$ negative bias against MOPITT at $500 \mathrm{hPa}$ in April in the NH (Shindell et al., 2006) and 5 to 40 ppbv negative bias against Arctic surface stations in the spring (Monks et al., 2015b). The model shows the best agreement in the $\mathrm{NH}$ tropics at this time of year.

TOMCAT CO concentrations in the SH in April are around $10-15$ ppbv larger than observed. Shindell et al. (2006) found good agreement between a 26-model ensemble mean at $500 \mathrm{hPa}$ compared to MOPITT, with individual models showing both negative and positive biases of between -15 and +15 ppbv, showing that the TOMCAT bias at this time of year is at the high end of the multi-model positive bias range.

The model negative bias in the $\mathrm{NH}$ and positive bias in the SH leads to a simulated interhemispheric gradient that is too low (see Fig. 5c), which is a common feature in chemistry models (Shindell et al., 2006). Several inverse modelling studies have suggested that wintertime $\mathrm{CO}$ emissions in the $\mathrm{NH}$ need to be increased in order to better match observations of CO (Pétron et al., 2004; Kopacz et al., 2010; Fortems-Cheiney et al., 2011). Transport errors in the model could also play a role; however, they are unlikely to cause such widespread biases of this magnitude in background $\mathrm{CO}$. In addition to this, as mentioned in Sect. 4.2, OH in TOMCAT is most likely too high at the surface, particularly in the tropics, and the $\mathrm{NH}: \mathrm{SH} \mathrm{OH}$ ratio is higher than estimates based on observations. This is likely to influence the lifetime of simulated $\mathrm{CO}$ and will contribute to the $\mathrm{NH}$ and $\mathrm{SH}$ biases. Strode et al. (2015) showed that by lowering the $\mathrm{NH}: \mathrm{SH} \mathrm{OH}$ ratio of current state-of-the-art models, simulations of $\mathrm{CO}$ can be improved. The cause of the lower simulated $\mathrm{NH}: \mathrm{SH}$ $\mathrm{OH}$ ratio in models is still unclear and may be linked to emission biases, where higher emissions of $\mathrm{CO}$ and VOCs in the $\mathrm{NH}$ may reduce $\mathrm{OH}$ concentrations, reducing the $\mathrm{NH}: \mathrm{SH}$ $\mathrm{OH}$ ratio.

In October, the interhemispheric gradient in $\mathrm{CO}$ is no longer as clear due to longer $\mathrm{CO}$ lifetimes in the $\mathrm{SH}$ and shorter lifetimes in the NH. This time of year is charac- 

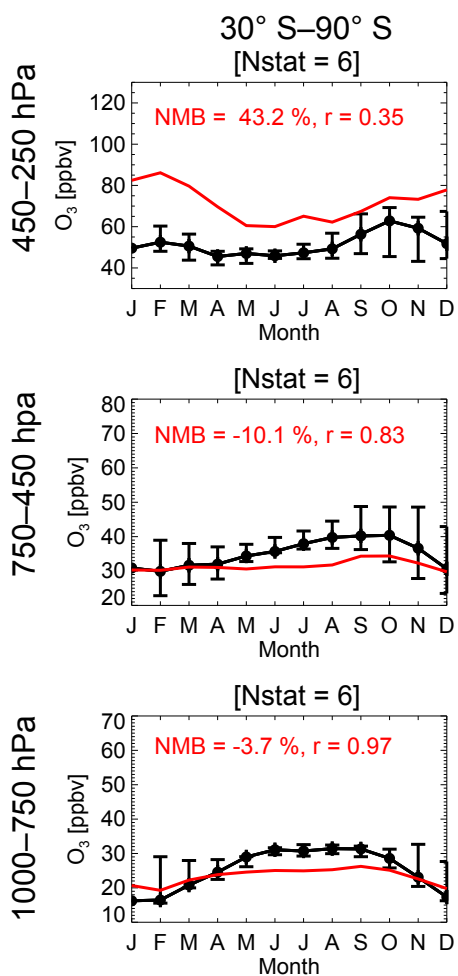
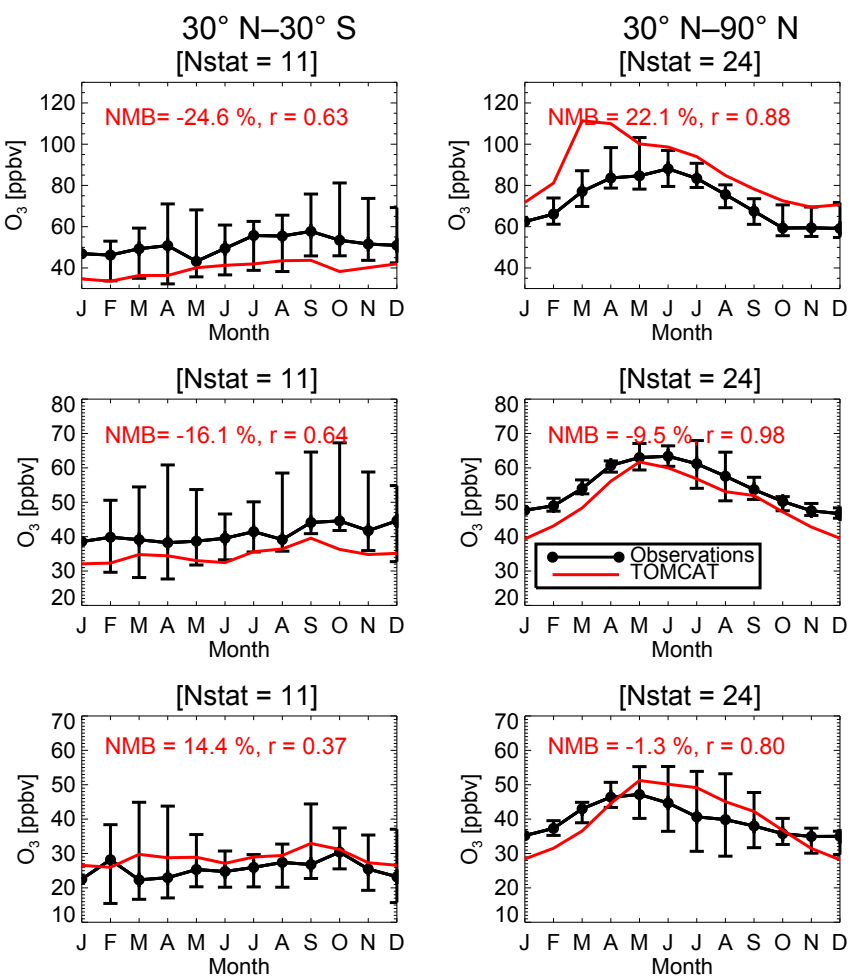

Figure 7. Median $\mathrm{O}_{3}$ concentrations (ppbv) taken from the Tilmes et al. (2012) ozonesonde climatology compared to TOMCAT-simulated concentrations. The data are averaged over three latitude ranges (left to right) and three pressure level ranges (top to bottom), where the error bars show the 25th and 75th percentiles of the observed concentrations and Nstat gives the number of sonde release sites located within each latitude range.

terised by peak fire emissions in the SH (van der Werf et al., 2010). For this reason, high concentrations of $\mathrm{CO}$ are seen by MOPITT over South America and there is a shift in the biomass burning emissions further south over Africa, resulting in higher $\mathrm{CO}$ over the Southern Ocean. TOMCAT also shows higher concentrations over the Southern Ocean due to the influence of fire emissions compared to April. However, fire emission location errors are clearly contributing to a mismatch between the $\mathrm{CO}$ plumes in the model and those seen by MOPITT. Total column CO over this region suggests that emissions from fires may be too large in the tropics, particularly over tropical Asia (not shown), and the fires are located too far north in Africa and too far west in South America, resulting in too much $\mathrm{CO}$ being transported out over the oceans in the tropics (see Fig. 5d and e). Naik et al. (2013) also showed that the ACCMIP multi-model annual mean simulated $\mathrm{CO}$ at $500 \mathrm{hPa}$ was $2-45 \mathrm{ppbv}$ too high compared to MOPITT in this region, supporting a high bias in $\mathrm{CO}$ fire emissions across different emission inventories in the $\mathrm{SH}$ and tropics at this time of year. Outside of the $10^{\circ} \mathrm{S}-$ $30^{\circ} \mathrm{N}$ region, the zonal mean $\mathrm{CO}$ shows much better agreement between TOMCAT and MOPITT than seen in April (see Fig. 5f).

Figure 6 compares simulated and measured $\mathrm{CO}$ at 14 different surface observatories that are located at several differ- ent latitudes and longitudes for the year 2008 (see Fig. 2a for station locations). TOMCAT generally captures the seasonal cycle, with high correlations values found at most stations (see $r$ values in Fig. 6). However, the amplitude of the seasonal cycle is less pronounced in the model in some regions. In agreement with the MOPITT comparison results, the model shows a large negative bias in winter and spring in the $\mathrm{NH}$, with particularly large biases at stations located at higher latitudes (Alert and Mace Head). This has been documented at Arctic surface sites previously (Shindell et al., 2008; Monks et al., 2015b). At latitudes $>25^{\circ} \mathrm{N}$ the model has a normalised mean bias (NMB) of between -14.6 and $-22.4 \%$. The model performs the best near the tropics, with NMBs of between -8.5 and $8.2 \%$. In the middle to high $\mathrm{SH}$ latitudes, the model overestimates $\mathrm{CO}$ concentrations throughout the year, with the largest biases occurring during the austral summer (NMBs of 21.2 to $28.5 \%$ ).

The 26-model study by Shindell et al. (2006) found that models have a negative bias between 20 and 80 ppbv at Alert in the Arctic during winter/spring and a more persistent positive bias throughout the year of up to $20-25 \mathrm{ppbv}$ at Cape Grim, exhibiting a transition from a negative bias in the $\mathrm{NH}$ to a positive bias in the SH that is similar to that found in TOMCAT. TOMCAT is within the bias range at Alert, with a winter negative bias of up to $\sim 50 \mathrm{ppbv}$, and at the upper 

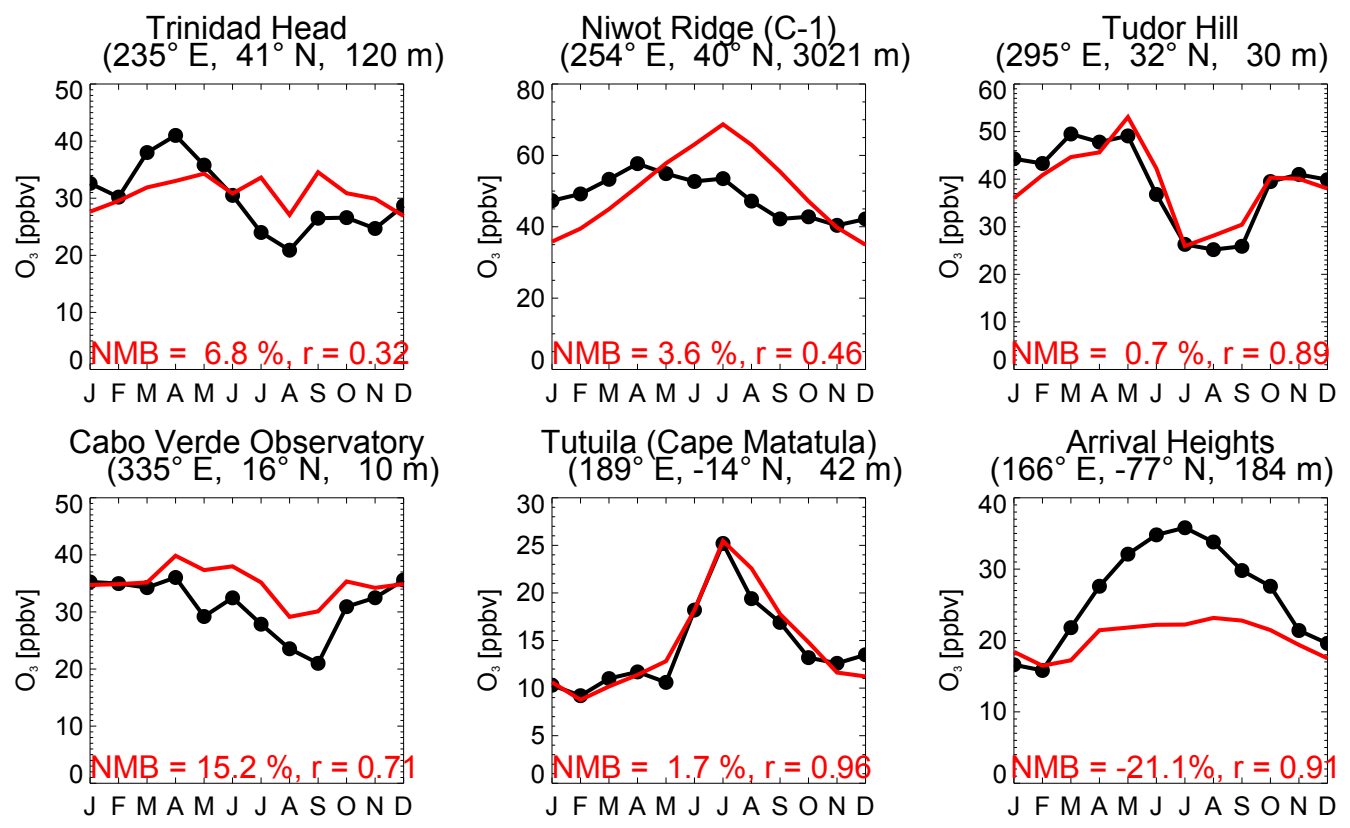

TOMCAT

Observations

Figure 8. Monthly mean 2008 observed and simulated $\mathrm{O}_{3}$ (ppbv) at several surface sites located throughout the globe. The panels are arranged by latitude from north to south, with Pearson correlation $(r)$ and normalised mean bias (NMB) between the observed and simulated monthly mean data printed on each panel.

(a) DJF

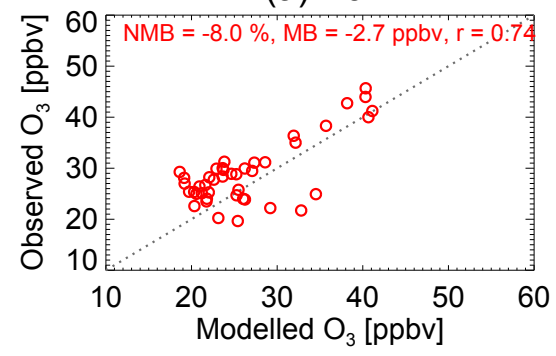

(c) JJA

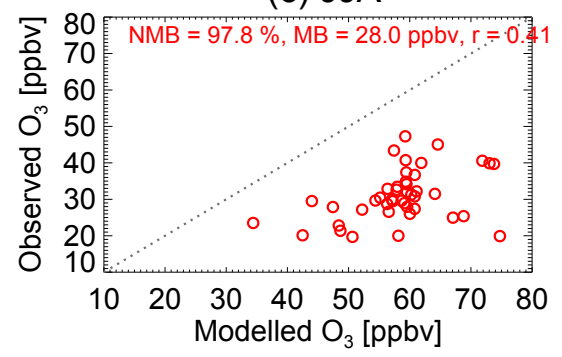

(b) MAM

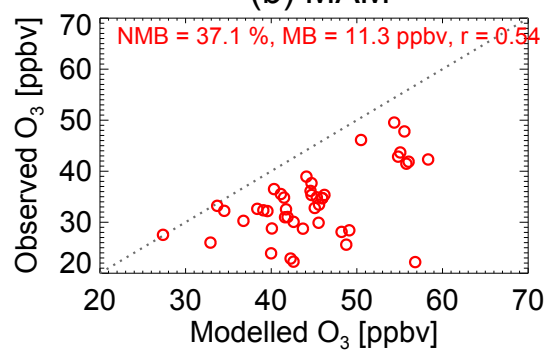

(d) SON

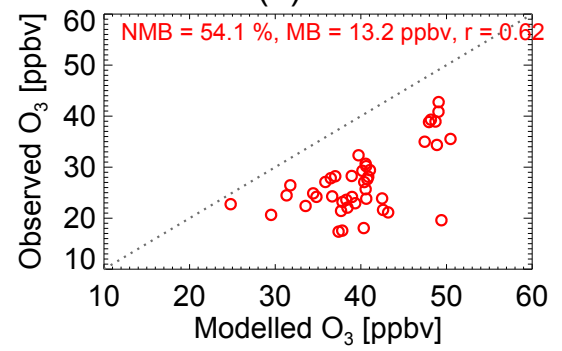

Figure 9. Scatter plots of seasonal mean observed and simulated $2008 \mathrm{O}_{3}$ concentrations (ppbv) at CASTNET EPA monitoring stations located in North America. 
(a) GOME-2 Ozone (DU) JJA
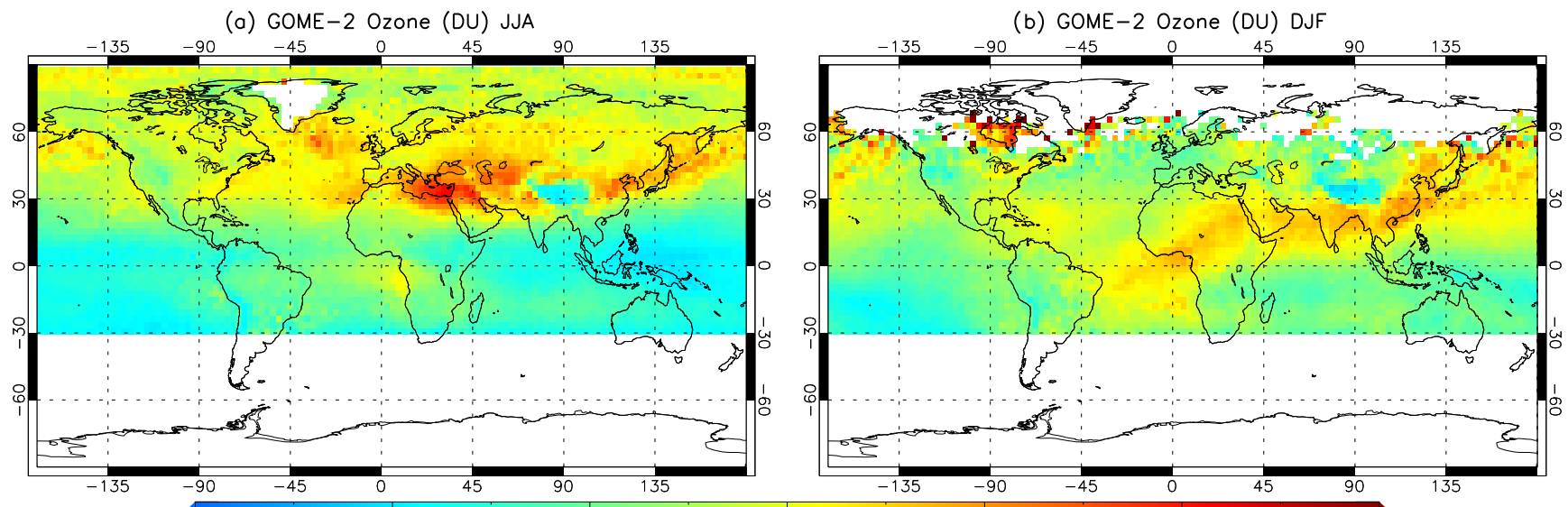

0.0

6.7

(c) TOMCAT - GOME-2 Ozone (DU) JJA

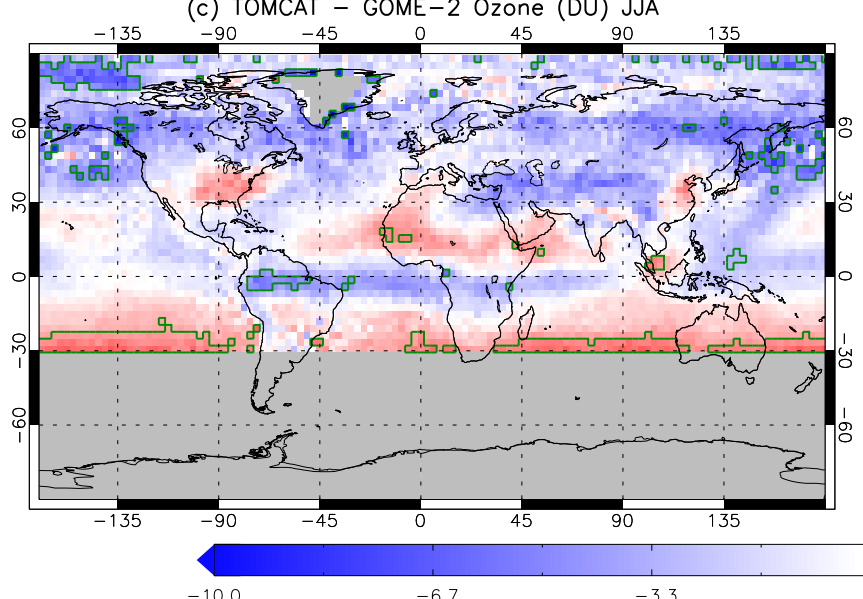

$20.0 \quad 26.7$

26.7
(d) TOMCAT 33.3
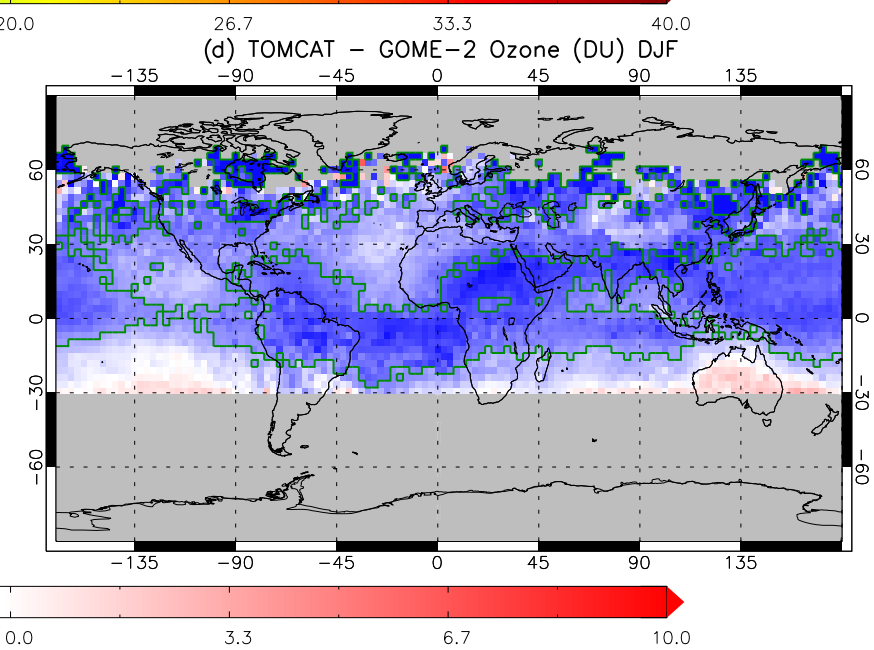

Figure 10. GOME-2 subcolumn $\mathrm{O}_{3}\left(0-6 \mathrm{~km}\right.$, DU) on the TOMCAT $2.8^{\circ} \times 2.8^{\circ}$ grid for (a) June-July-August 2008 (JJA) and (b) DecemberJanuary-February 2008 (DJF). Panels (c) and (d) show the difference in concentrations between TOMCAT and GOME-2. The green polygons indicate where the mean bias $(\mathrm{MB})$ is greater than the satellite error.

end of the bias range at Cape Grim, with up to $\sim 25 \mathrm{ppbv}$ at Cape Grim. The surface and MOPITT comparisons show that these model biases exist at the surface and throughout the free troposphere, and are generally consistent with biases found in other chemical transport models.

\subsection{Evaluation of ozone}

Ozonesonde data are compared to simulated $\mathrm{O}_{3}$ in Fig. 7 . The data have been separated into three different altitude and latitude bands. The model overestimates $\mathrm{O}_{3}$ at higher $\mathrm{NH}$ and SH latitudes in the highest altitude band (NMB of 22 to $43.2 \%$ ), possibly due to too much downward mixing of stratospheric $\mathrm{O}_{3}$ in the model at these altitudes. TOMCAT also overestimates $\mathrm{O}_{3}$ at the surface in the tropics (NMB of 14.4 to $16.7 \%$ ), but the model lies within the range of observations. Elsewhere, the model has a negative bias (NMB of -1.2 to $-24.6 \%$ ) but lies within the range of observations at several times of the year. Most of the negative bias in the higher latitudes is being driven by wintertime underestimates in $\mathrm{O}_{3}$ in both the $\mathrm{SH}$ and $\mathrm{NH}$. Young et al. (2013) found that the multi-model ACCMIP mean $\mathrm{O}_{3}$ is also negatively biased in the SH during the winter months when compared to the same data, suggesting that this is a common feature in chemistry models. However, they found that the ACCMIP models overestimated $\mathrm{O}_{3}$ in the $\mathrm{NH}$ high latitudes during winter.

The low TOMCAT bias in wintertime $\mathrm{O}_{3}$ can also be seen in surface data located at high latitudes in the $\mathrm{SH}$ at Arrival Heights (see Fig. 8), where TOMCAT has a negative $\mathrm{O}_{3}$ bias of $\sim 10-15 \mathrm{ppbv}$ during the $\mathrm{SH}$ austral winter (NMB of $-21.1 \%$ ). This suggests that the model may have difficulties reproducing $\mathrm{O}_{3}$ photochemistry in the winter in remote, dark and cold regions or the model may deposit too much $\mathrm{O}_{3}$ onto snow/ice covered surfaces. Whilst most models in the POLMIP study were also negatively biased at the Summit observatory in the Arctic during winter, TOMCAT simulated some of the lowest concentrations (Monks et al., 2015b). Outside of the poles, the model simulates concentrations of $\mathrm{O}_{3}$ that are in much better agreement with the obser- 
vations (NMB of 0.7 to $15.2 \%$ ). In the NH during the summer, TOMCAT tends to overestimate concentrations. This is a common feature in models in the $\mathrm{NH}$ during summer, which has been identified to be particularly pronounced over the eastern US (e.g. Ellingsen et al., 2008; Fiore et al., 2009; $\mathrm{Yu}$ et al., 2010). $\mathrm{O}_{3}$ at the surface is also compared to data from 44 EPA CASTNET stations located in the US (Fig. 9). This high summer bias over the US is clearly evident, with a large mean bias (MB) of $28 \mathrm{ppbv}$ (NMB of $97.8 \%$ ). The best agreement is seen in winter (MB of $-2.7 \mathrm{ppbv}, \mathrm{NMB}$ of $-8 \%$ ). ValMartin et al. (2014) showed that model summertime $\mathrm{O}_{3}$ biases could be reduced from 44 to $28 \%$ over the US and from 25 to $14 \%$ over Europe when improvements were made to a coupled land-atmosphere model's deposition scheme. This suggests that using a more sophisticated deposition scheme coupled to a land model may improve TOMCAT simulations of summertime $\mathrm{O}_{3}$.

Subcolumn $\mathrm{O}_{3}$ between 0 and $6 \mathrm{~km}$ (up to $\sim 500 \mathrm{hPa}$ ) is compared to GOME-2 retrievals in Fig. 10. MB errors that are greater than the satellite error are highlighted with green polygons. In DJF, GOME-2 measures the highest concentrations of $\mathrm{O}_{3}(\sim 25 \mathrm{DU})$ in regions near $\mathrm{O}_{3}$ precursor emissions and those with enough sunlight to initiate photochemistry at this time of year (e.g. India, China and northern Africa; Fig. 10b). TOMCAT shows negative MBs of up to $-10 \mathrm{DU}$ in several regions, with some of the larger biases being co-located with high observed $\mathrm{O}_{3}$ concentrations (see Fig. 10d). Comparisons to ozonesondes (see Fig. 7) further support this and show that the model $\mathrm{O}_{3}$ may be biased low (by 5-10 ppbv) in the tropical region at this time of year at altitudes between 750 and $450 \mathrm{hPa}$ (although the model does lie within the ozonesonde observed ranges). In JJA, the model bias is much smaller with very few significant MBs being highlighted (see Fig. 10c). There is evidence that the model overestimates $\mathrm{O}_{3}$ at this time of year over the southeast US, in agreement with the CASTNET modelobservation comparisons, as well as some evidence that $\mathrm{O}_{3}$ is also overestimated near Cabo Verde off the coast of Africa, as seen in Fig. 8.

\subsection{Evaluation of VOCs}

In Fig. 11, measurements of ethene, ethane, propene, propane, toluene and butane are compared to simulated concentrations at the mountain site in Hohenpeissenberg, Germany. The observations show a seasonal cycle that is particularly pronounced for ethene, ethane, propene and toluene, with peak concentrations in winter and spring, when $\mathrm{OH}$ concentrations are lower and the lifetimes of VOCs are longer, and a minimum in summer. For ethane and propane, the model captures the seasonal transitions ( $r$ values of 0.94 and 0.99 , respectively) but shows a much smaller amplitude due to large negative biases, particularly in winter (NMB of -31.3 and $-25.8 \%$, respectively). This can also be seen at high latitudes over North America throughout the tropo-

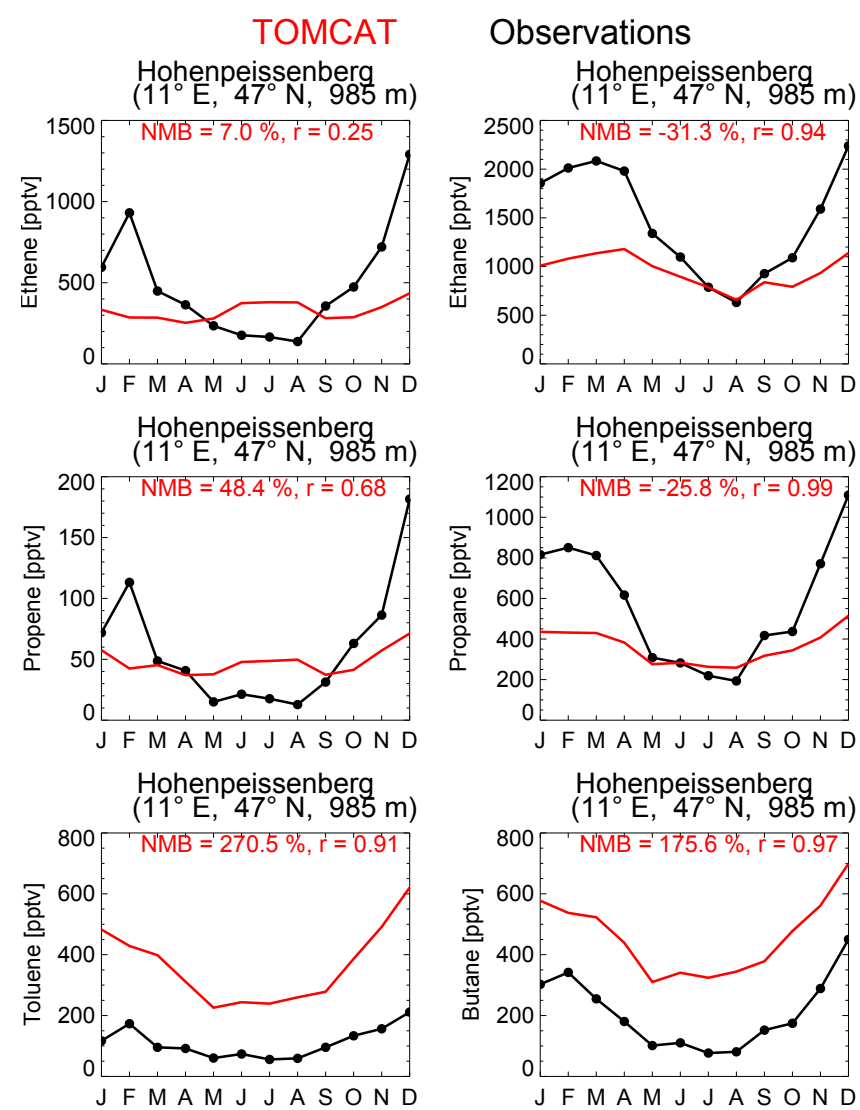

Figure 11. Monthly mean 2008 observed and simulated VOCs (pptv) at the European high-altitude observatory, Hohenpeissenberg. Pearson correlation $(r)$ and normalised mean bias (NMB) between the observed and simulated monthly mean data are printed on each panel.

sphere when compared to the ARCTAS data in Fig. 12. It has been suggested previously that the current anthropogenic emissions of ethane are too low in global models (Franco et al., 2016; Tilmes et al., 2016), which is likely to explain the negative bias in TOMCAT ethane. Propane is also shown to be too low throughout the tropospheric column (Fig. 12) and has also been found to be biased low in the $\mathrm{NH}$ across different models, suggesting a similar problem with emissions (Emmons et al., 2015; Tilmes et al., 2015). For ethene and propene, the seasonal cycle is not well captured by the model due to enhancements in summer $(r=0.25-0.68)$. This is likely to be due to incorrect local emissions at this time of year (from biogenic or fire sources) or difficulties capturing local turbulent transport at this mountain site, which is a common problem in models (Zhang et al., 2008; Feng et al., 2011). Similar to ethane and propane, the model also shows negative biases that are particularly large in winter, suggesting underestimated anthropogenic emissions in the NH. For toluene and butane, the model captures the seasonal cycle well ( $r$ values of 0.91 and 0.97 , respectively), but some large 

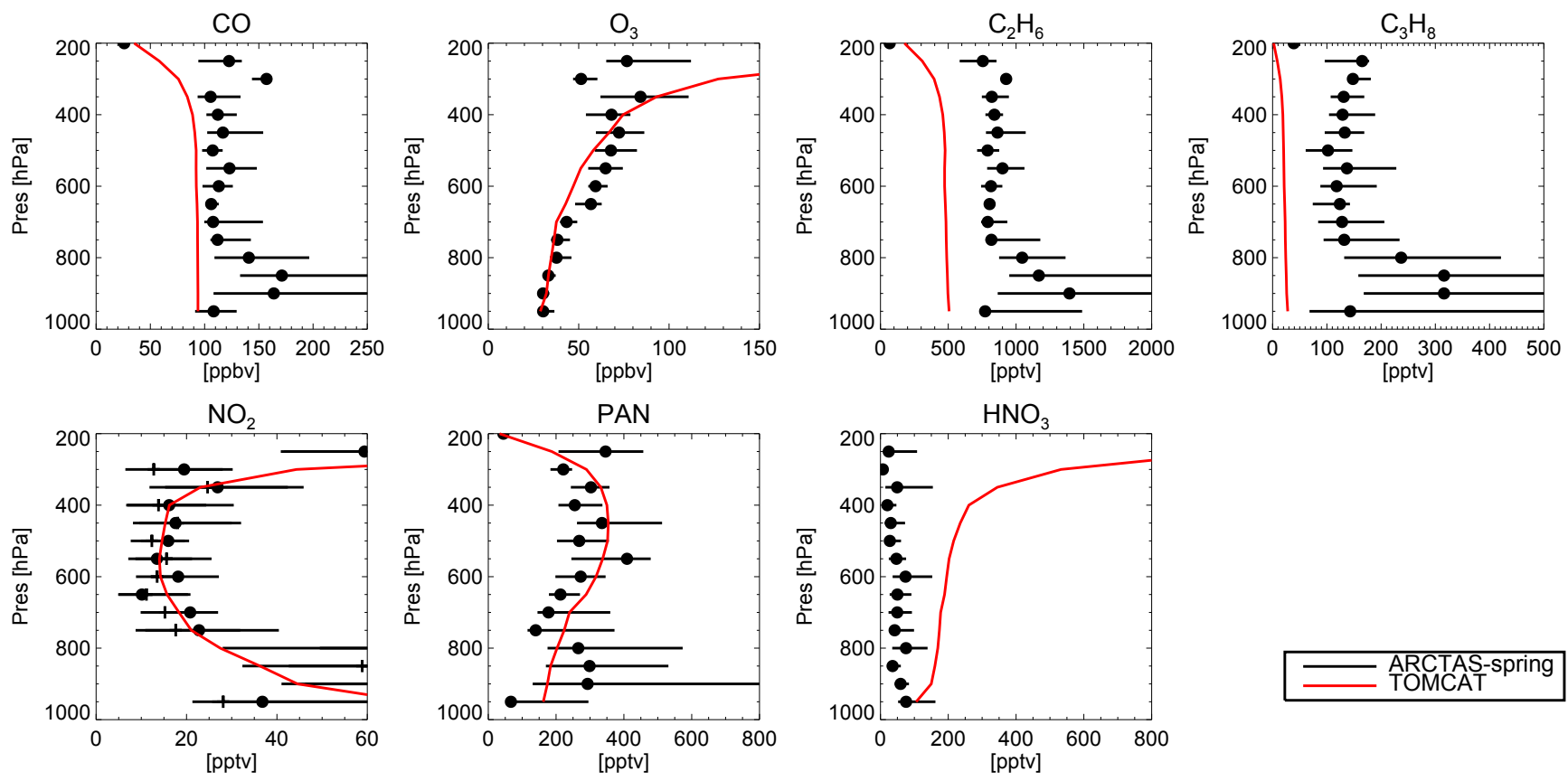

Figure 12. Comparison of simulated and observed concentrations of $\mathrm{CO}, \mathrm{O}_{3}, \mathrm{C}_{2} \mathrm{H}_{6}, \mathrm{C}_{3} \mathrm{H}_{8}, \mathrm{NO}_{2}, \mathrm{PAN}_{\text {and }} \mathrm{HNO}_{3}$ for the ARCTAS July 2008 flights (seven flights).
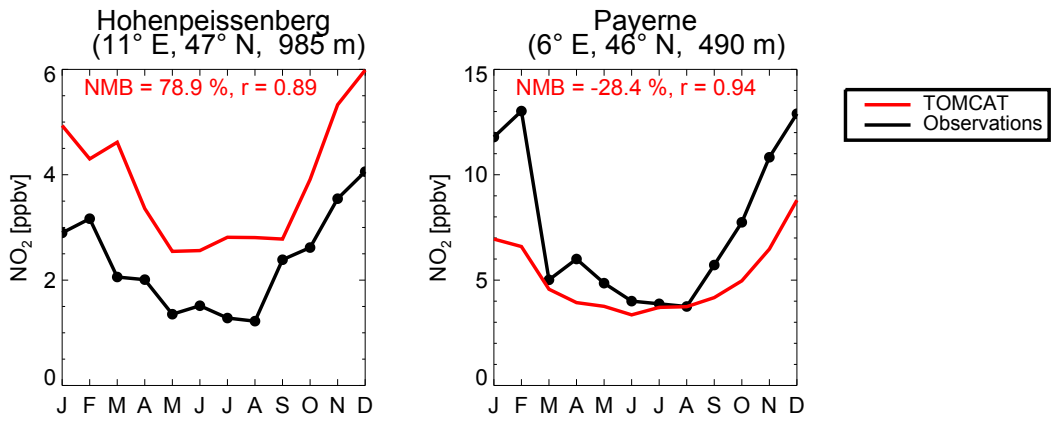

Figure 13. Monthly mean 2008 observed and simulated $\mathrm{NO}_{2}$ (ppbv) at two European surface sites located in Europe. Pearson correlation ( $r$ ) and normalised mean bias (NMB) between the observed and simulated monthly mean data are printed on each panel.

positive biases are found consistently throughout the year (NMBs of 270.5 and $175.6 \%$, respectively).

\subsection{Evaluation of reactive nitrogen}

Oxides of nitrogen $\left(\mathrm{NO}_{y}\right)$ are important atmospheric pollutants and are key in the production of $\mathrm{O}_{3}$. In addition, speciation of $\mathrm{NO}_{y}$ is dependent on oxidative capacity, organic chemistry and heterogeneous chemistry. Hence, evaluation of speciated $\mathrm{NO}_{y}$ is a valuable test of several interrelated aspects of model chemistry. Here, we use observations of $\mathrm{NO}_{2}$, nitric acid $\left(\mathrm{HNO}_{3}\right)$ and PAN to evaluate the model $\mathrm{NO}_{y}$.

In Fig. 13, 2008 measurements of $\mathrm{NO}_{2}$ from two European observatories are compared to simulated concentrations (see Fig. 2c for locations). Observed concentrations at both Hohenpeissenberg and Payerne show a minimum in summer and a maximum in winter, with the model capturing the seasonal cycle well, suggesting that the model is able to reproduce seasonal changes in photochemistry ( $r$ values of 0.89 and 0.94 , respectively). However, TOMCAT overestimates the concentrations at Hohenpeissenberg throughout the year (NMB of $78.9 \%$ ) but underestimates concentrations at Payerne (NMB of $-28.4 \%$ ). The model shows only marginally higher concentrations at Payerne compared to Hohenpeissenberg. The observations show that this difference is larger in reality, suggesting a higher gradient in concentrations between the two different stations both horizontally and vertically. As $\mathrm{NO}_{2}$ is short-lived, it is difficult for global models to reproduce observations due to coarse horizontal and vertical resolutions, which is likely to affect the model's ability to capture concentration gradients (Huijnen et al., 2010). The model is able to capture the changes in $\mathrm{NO}_{2}$ with altitude and 
(a) OMI $\mathrm{NO}_{2}\left(10^{15}\right.$ molecules $\left.\mathrm{cm}^{-2}\right) \mathrm{JJA}$

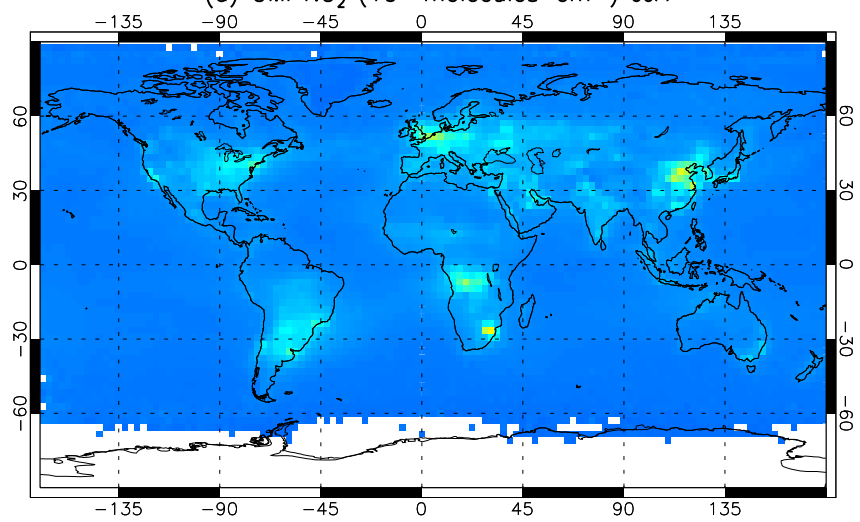

(c) TOMCAT - OMI NO$\left(10^{15}\right.$ molecules $\left.\mathrm{cm}^{-2}\right)$ JJA

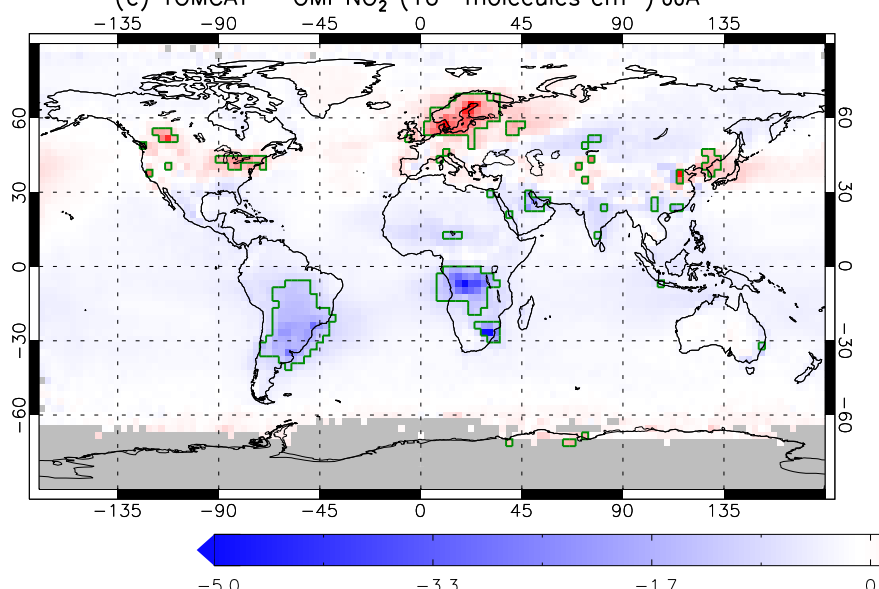

$-5.0$

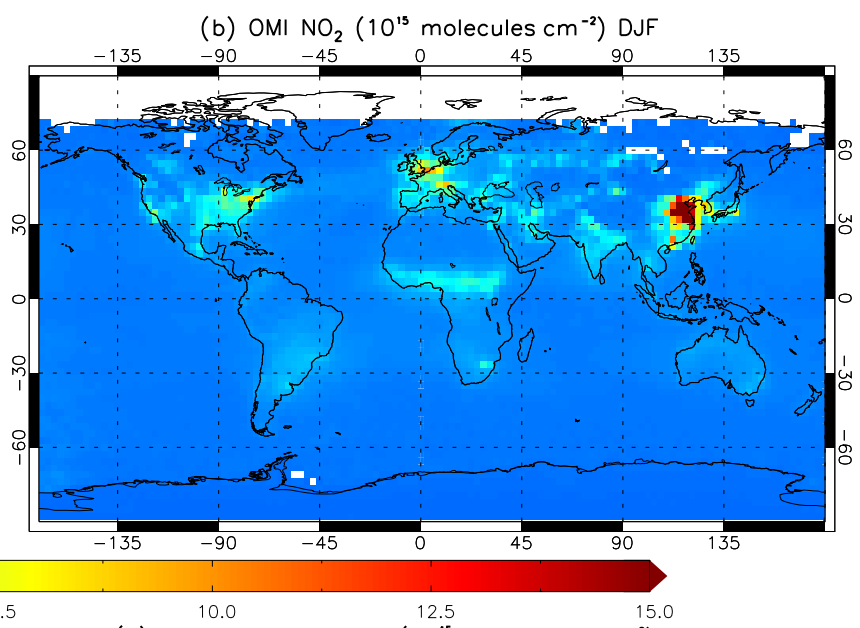

(b) OMI NO $2\left(10^{15}\right.$ molecules $\left.\mathrm{cm}^{-2}\right)$ DJF

(d) TOMCAT - OMI NO $2\left(10^{15}\right.$ molecules $\left.\mathrm{cm}^{-2}\right)$ DJF

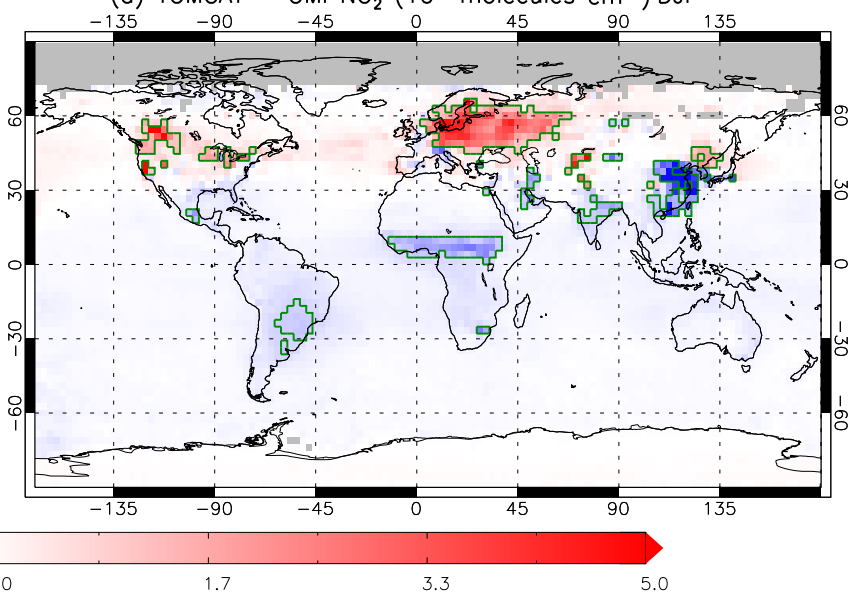

Figure 14. 2008 tropospheric $\mathrm{NO}_{2}$ column $\left(\times 10^{15}\right.$ molecules $\left.\mathrm{cm}^{-2}\right)$ from OMI on the TOMCAT model $2.8^{\circ} \times 2.8^{\circ}$ grid for (a) JuneJuly-August and (b) December-January-February, along with the TOMCAT OMI tropospheric column $\mathrm{NO}_{2}$ mean bias (MB) for the same periods (c, d). The green polygons are where the absolute MB is greater than the satellite error.

captures the magnitude well in the middle troposphere (see Fig. 12).

Figure 14 shows 2008 DJF and JJA OMI satellite $\mathrm{NO}_{2}$ column data alongside the TOMCAT MB. Due to the short lifetime of $\mathrm{NO}_{2}$, high concentrations are observed near emission regions. In the $\mathrm{NH}$, high concentrations are seen over Asia, North America and Europe, near some of the largest anthropogenic emission sources. In both seasons, the model simulates concentrations that are too high over parts of Europe. This is likely to be linked to emissions due to the short lifetime of $\mathrm{NO}_{2}$. Due to the location near the Baltic and North seas, this could indicate that ship emissions are too large in this region. Large negative biases in $\mathrm{NO}_{2}$ near China are seen in the model in the $\mathrm{NH}$ winter. This has been seen in several models previously when comparing to OMI and is thought to be due to anthropogenic emissions that are too low (Emmons et al., 2015). In contrast, TOMCAT has a positive model bias in this region during summer, most likely due to the FINN fire emissions being too high, which has also been seen in multiple models being compared to OMI (Emmons et al., 2015).

In the SH, OMI observes the largest concentrations over the high-biomass-burning regions of South America, Africa and Australia. In these regions, the model shows $\mathrm{NO}_{2}$ concentrations that are too low during both seasons, suggesting FINN fire emissions are too low in the SH. This is in contrast to CO satellite comparisons, which suggested fire emissions are too high in this region (see Sect. 4.3). This therefore indicates that emission factors used to calculate fire emissions need to be further evaluated in the tropics and the $\mathrm{SH}$.

Figure 15 shows PAN comparisons at the mountain site in Schauinsland, Germany. Observations at this location show concentrations that peak in April, with a winter minimum. TOMCAT concentrations peak later in the year in June but capture the strong drop in concentrations leading towards a winter minimum $(r=0.82)$. Simulated concentrations show reasonable agreement with the observations (NMB of $6.9 \%$ ). PAN during the summer months shows reasonable agreement 


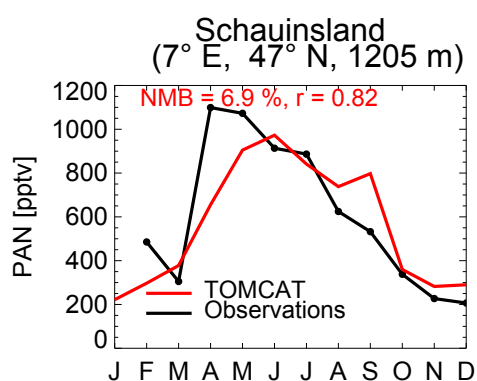

Figure 15. Monthly mean 2008 observed and simulated PAN (pptv) at the Schauinsland mountain observatory in Germany.

with aircraft data shown in Fig. 12. However, $\mathrm{HNO}_{3}$ is overestimated in the model, possibly due to too much production or not enough washout. In an Arctic model intercomparison project (POLMIP), TOMCAT had some of the highest concentrations of PAN and $\mathrm{HNO}_{3}$ compared to other models (Emmons et al., 2015), suggesting that TOMCAT $\mathrm{NO}_{y}$ production is higher and/or loss may be lower compared to other models. The observations at Schauinsland suggest that TOMCAT may do a better job in simulating PAN over Europe at lower latitudes. In addition to this, Pope et al. (2016) found that TOMCAT PAN overestimated upper tropospheric Michelson Interferometer for Passive Atmospheric Sounding (MIPAS) PAN at altitudes above $200 \mathrm{hPa}$ in winter and spring. Due to the importance of reactive nitrogen in $\mathrm{O}_{3}$ production, there is a need for further investigation and evaluation of these species in the future when more recent observations become available.

\section{Summary}

This paper describes the TOMCAT 3-D chemical transport model's tropospheric chemistry scheme. The current scheme has a more detailed representation of hydrocarbon chemistry compared to the previously documented version found in Arnold et al. (2005). The current scheme includes the degradation of ethene, propene, toluene and butane based on the Extended Tropospheric Chemistry scheme and monoterpene chemistry based on MOZART-3 chemistry. A 1-year simulation for the year 2008 is used to document model performance against a range of surface, satellite, aircraft and balloon measurements. The model is generally able to capture the main spatial and seasonal features of high and low concentrations of $\mathrm{CO}, \mathrm{O}_{3}$, VOCs and reactive nitrogen. However, several negative and positive biases are present in TOMCAT during certain times of the year and at certain locations. Some of these biases are prevalent in current state-of-the-art chemistry models, but some biases that are specific to TOMCAT are also highlighted.

TOMCAT global mean tropospheric OH $(1.08 \times$ $10^{6}$ molecules $\mathrm{cm}^{-3}$ ) is higher than estimates inferred from MCF observations $\left(0.94-1.0 \times 10^{6}\right.$ molecules $\left.\mathrm{cm}^{-3}\right)$. How- ever, this is a common feature across chemistry models, and the TOMCAT global mean $\mathrm{OH}$ is at the lower end of concentrations reported in previous multi-model intercomparison projects $\left(1.08-1.17 \times 10^{6}\right.$ molecules $\left.\mathrm{cm}^{-3}\right)$. TOMCAT has the highest concentrations (in molecules $\mathrm{cm}^{-3}$ ) of $\mathrm{OH}$ in the lower tropical troposphere, which is in contrast to the ACCMIP multi-model mean $\mathrm{OH}$, which has the highest $\mathrm{OH}$ concentrations in the tropical upper troposphere. Observationally constrained $\mathrm{OH}$ shows the highest concentrations of $\mathrm{OH}$ in the middle tropical troposphere suggesting that TOMCAT has too much $\mathrm{OH}$ at the surface in the tropics. In addition to this, TOMCAT has a higher $\mathrm{NH}: \mathrm{SH} \mathrm{OH}$ ratio (1.37) compared to the ratio inferred from $\mathrm{MCF}$ observations (0.98), which is again a common feature in chemistry models, with TOMCAT being at the upper limit of the multi-model mean value calculated from the ACCMIP models $(1.28 \pm 0.1)$. This suggests that simulated $\mathrm{OH}$ in current chemistry models is largely uncertain and more work is needed to understand the cause of the lower simulated $\mathrm{NH}: \mathrm{SH} \mathrm{OH}$ ratio in models. One possibility could be underestimated emissions in the $\mathrm{NH}$ which may be contributing to $\mathrm{OH}$ concentrations being too high in this region.

TOMCAT CO is negatively biased during winter and spring in the NH when compared to MOPITT and surface observations. In contrast, $\mathrm{CO}$ is positively biased throughout the year in the SH. The negative bias in the $\mathrm{NH}$ is a common feature in chemistry models and TOMCAT lies well within the range of biases found in other models. The TOMCAT SH positive bias is at the upper range of positive biases reported in other models, with some models reporting negative biases. Underestimated emissions in the $\mathrm{NH}$ are thought to play a role in the negative $\mathrm{NH} \mathrm{CO}$ bias, whilst comparisons with MOPITT suggest that TOMCAT fire emissions may be too high in the $\mathrm{SH}$, contributing to the model positive bias. $\mathrm{OH}$ biases could also play a role in the $\mathrm{CO}$ bias, in particular in the $\mathrm{NH}$ where near-surface $\mathrm{OH}$ is around $50 \%$ larger than methyl chloroform-constrained $\mathrm{OH}$ estimates. Lower $\mathrm{OH}$ concentrations in the model at the surface would lead to an increase in $\mathrm{CO}$ concentrations in the $\mathrm{NH}$ and would also reduce the $\mathrm{NH}$ : $\mathrm{SH} \mathrm{OH}$ ratio.

TOMCAT is able to capture the seasonality of $\mathrm{O}_{3}$ in most locations, with the model lying within the range of observations made during balloon soundings during most times of the year. The notable exceptions to this are (1) at high latitudes during winter conditions, where TOMCAT simulates $\mathrm{O}_{3}$ that is negatively biased by up to $15 \mathrm{ppbv}$ when compared to both surface and ozonesonde measurements and (2) in the $\mathrm{NH}$ during summer, where TOMCAT is positively biased by up to $28 \mathrm{ppbv}$ over North America when compared to surface sites. GOME-2 satellite data show that model performance is better in JJA compared to DJF, where the model underestimates $\mathrm{O}_{3}$ by up to $10 \mathrm{DU}$ in regions with high observed $\mathrm{O}_{3}$ concentrations near Asia and Africa. 
VOC surface measurements show large negative biases in simulated winter/spring $\mathrm{C}_{2}-\mathrm{C}_{3}$ alkanes and alkenes, which is likely driven by underestimated anthropogenic emissions. This has been seen previously for ethane and propane in several models in the $\mathrm{NH}$.

TOMCAT captures the rapid decline in PAN concentrations between summer and winter at a European mountain site but simulates peak PAN concentrations in June rather than in April. TOMCAT is able to capture the seasonal cycle of $\mathrm{NO}_{x}$ well at two European surface sites but has trouble capturing the concentrations, overestimating them at the lower altitude Payerne site and underestimating them at the higher altitude site of Hohenpeissenberg. This is likely to be at least partly due to the very short lifetime of $\mathrm{NO}_{x}$ and the coarse model grid. Tropospheric satellite $\mathrm{OMI} \mathrm{NO}_{2}$ showed regional differences in TOMCAT biases, with negative biases existing over China in DJF (possibly due to anthropogenic emissions) and South America and Africa (possibly due to fire emissions), and positive biases over Europe in DJF and JJA. The biases over Asia have been shown to exist in several other models when using the same emissions as used here. In addition to this, models have been shown previously to vary widely in the simulation of species such as $\mathrm{HNO}_{3}, \mathrm{PAN}$ and acetaldehyde. Therefore, observations of these species that are collected continuously throughout the year at several locations globally would be valuable in evaluating chemical transport models in the future and understanding model biases in $\mathrm{O}_{3}$.

Code availability. TOMCAT/SLIMCAT (www.see.leeds.ac.uk/ tomcat) is a UK community model. It is available to UK (or NERC-funded) researchers who normally access the model on common facilities or who are helped to install it on their local machines. As it is a complex research tool, new users will need help to use the model optimally. We do not have the resources to release and support the model in an open way. Any potential user interested in the model should contact Martyn Chipperfield. The model updates described in this paper are included in the standard model library.

\section{The Supplement related to this article is available online at https://doi.org/10.5194/gmd-10-3025-2017- supplement.}

Competing interests. The authors declare that they have no conflict of interest.

Acknowledgements. Funding for this work was provided by the NERC EurEx project (NE/H020241/1) and TOMCAT model simulations were performed on the UK Archer HPC system. We would like to thank the many providers of observational data that have been used in this paper. Specifically, we thank the World Ozone and Ultraviolet Data Centre (WOUDC), the National
Oceanic and Atmospheric Administration Earth System Research Laboratory, Global Monitoring Division (NOAA ESRL, GMD) and the Southern Hemisphere ADditional OZonesondes (SHADOZ) for data that were used in the ozonesonde climatology. We also thank the MOPITT team that provided $\mathrm{CO}$ satellite data and Louisa K. Emmons for providing code to process the MOPITT data; the TEMIS website and KNMI OMI satellite group for use of tropospheric $\mathrm{NO}_{2}$ column data; the ARCTAS DC-8 team who provided aircraft measurements; the United States Environmental Protection Agency (EPA) Clean Air Status and Trends Network (CASTNET) for $\mathrm{O}_{3}$ surface measurements; and the Global Atmosphere Watch (GAW) program for the use of the World Data Centre for Greenhouse Gases (WDCGG), which provided surface measurements of $\mathrm{CO}, \mathrm{O}_{3}, \mathrm{NO}_{x}, \mathrm{PAN}$ and VOCs. The WDCGG providers whose data were used include NOAA/ESRL, University of York, UBA, Empa, RIVM and DWD.

Edited by: Slimane Bekki

Reviewed by: four anonymous referees

\section{References}

Archibald, A. T., Cooke, M. C., Utembe, S. R., Shallcross, D. E., Derwent, R. G., and Jenkin, M. E.: Impacts of mechanistic changes on $\mathrm{HO}_{x}$ formation and recycling in the oxidation of isoprene, Atmos. Chem. Phys., 10, 8097-8118, https://doi.org/10.5194/acp-10-8097-2010, 2010.

Archibald, A. T., Levine, J. G., Abraham, N. L., Cooke, M. C., Edwards, P. M., Heard, D. E., Jenkin, M. E., Karunaharan, A., Pike, R. C., Monks, P. S., Shallcross, D. E., Telford, P. J., Whalley, L. K., and Pyle, J. A.: Impacts of HOx regeneration and recycling in the oxidation of isoprene: Consequences for the composition of past, present and future atmospheres, Geophys. Res. Lett., 38, 105804, https://doi.org/10.1029/2010GL046520, 2011.

Arnold, S. R., Chipperfield, M. P., and Blitz, M. A.: A threedimensional model study of the effect of new temperaturedependent quantum yields for acetone photolysis, J. Geophys. Res., 110, D22305, https://doi.org/10.1029/2005JD005998, 2005.

Atkinson, R. and Arey, J.: Gas-phase tropospheric chemistry of biogenic volatile organic compounds: a review, Atmos. Environ., 37, 197-219, https://doi.org/10.1016/S1352-2310(03)00391-1, 2003.

Atkinson, R., Baulch, D. L., Cox, R. A., Crowley, J. N., Hampson, R. F., Hynes, R. G., Jenkin, M. E., Rossi, M. J., and Troe, J.: IUPAC Subcommittee for Gas Kinetic Data Evaluation. Summary of evaluated kinetic data and photochemical data for atmospheric chemistry, available at: http://www.iupac-kinetic.ch.cam.ac.uk/, last access: 1 January 2004a.

Atkinson, R., Baulch, D. L., Cox, R. A., Crowley, J. N., Hampson, R. F., Hynes, R. G., Jenkin, M. E., Rossi, M. J., and Troe, J.: Evaluated kinetic and photochemical data for atmospheric chemistry: Volume I - gas phase reactions of $\mathrm{O}_{x}, \mathrm{HO}_{x}$, $\mathrm{NO}_{x}$ and $\mathrm{SO}_{x}$ species, Atmos. Chem. Phys., 4, 1461-1738, https://doi.org/10.5194/acp-4-1461-2004, 2004b.

Atkinson, R., Baulch, D. L., Cox, R. A., Crowley, J. N., Hampson, R. F., Hynes, R. G., Jenkin, M. E., Rossi, M. J., and Troe, J.: IUPAC Subcommittee for Gas Kinetic Data Evaluation. Summary 
of evaluated kinetic data and photochemical data for atmospheric chemistry, available at: http://www.iupac-kinetic.ch.cam.ac.uk/, last access: 1 January 2005.

Atkinson, R., Baulch, D. L., Cox, R. A., Crowley, J. N., Hampson, R. F., Hynes, R. G., Jenkin, M. E., Rossi, M. J., and Troe, J.: IUPAC Subcommittee for Gas Kinetic Data Evaluation. Summary of evaluated kinetic data and photochemical data for atmospheric chemistry, available at: http://www.iupac-kinetic.ch.cam.ac.uk/, last access: 1 January 2006a.

Atkinson, R., Baulch, D. L., Cox, R. A., Crowley, J. N., Hampson, R. F., Hynes, R. G., Jenkin, M. E., Rossi, M. J., Troe, J., and Subcommittee, I.: Evaluated kinetic and photochemical data for atmospheric chemistry: Volume II - gas phase reactions of organic species, Atmos. Chem. Phys., 6, 3625-4055, https://doi.org/10.5194/acp-6-3625-2006, 2006b.

Berntsen, T., Fuglestvedt, J., Joshi, M., Shine, K., Stuber, N., Ponater, M., Sausen, R., Hauglustaine, D., and Li, L.: Response of climate to regional emissions of ozone precursors: sensitivities and warming potentials, Tellus B, 57, 283-304, https://doi.org/10.1111/j.1600-0889.2005.00152.x, 2005.

Bloss, W. J., Evans, M. J., Lee, J. D., Sommariva, R., Heard, D. E., and Pilling, M. J.: The oxidative capacity of the troposphere: Coupling of field measurements of $\mathrm{OH}$ and a global chemistry transport model, Faraday Discuss., 130, 425-436, https://doi.org/10.1039/B419090D, 2005.

Boersma, K., Jacob, D., Bucsela, E., Perring, A., Dirksen, R., van der A, R., Yantosca, R., Park, R., Wenig, M., Bertram, T., and Cohen, R.: Validation of OMI tropospheric $\mathrm{NO}_{2}$ observations during INTEX-B and application to constrain emissions over the eastern United States and Mexico, Atmos. Environ., 42, 44804497, https://doi.org/10.1016/j.atmosenv.2008.02.004, 2008.

Boersma, K. F., Eskes, H. J., Veefkind, J. P., Brinksma, E. J., van der A, R. J., Sneep, M., van den Oord, G. H. J., Levelt, P. F., Stammes, P., Gleason, J. F., and Bucsela, E. J.: Near-real time retrieval of tropospheric $\mathrm{NO}_{2}$ from OMI, Atmos. Chem. Phys., 7, 2103-2118, https://doi.org/10.5194/acp-7-2103-2007, 2007.

Boersma, K. F., Eskes, H. J., Dirksen, R. J., van der A, R. J., Veefkind, J. P., Stammes, P., Huijnen, V., Kleipool, Q. L., Sneep, M., Claas, J., Leitão, J., Richter, A., Zhou, Y., and Brunner, D.: An improved tropospheric $\mathrm{NO}_{2}$ column retrieval algorithm for the Ozone Monitoring Instrument, Atmos. Meas. Tech., 4, 19051928, https://doi.org/10.5194/amt-4-1905-2011, 2011.

Bousquet, P., Hauglustaine, D. A., Peylin, P., Carouge, C., and Ciais, P.: Two decades of $\mathrm{OH}$ variability as inferred by an inversion of atmospheric transport and chemistry of methyl chloroform, Atmos. Chem. Phys., 5, 2635-2656, https://doi.org/10.5194/acp-5-2635-2005, 2005.

Braak, R.: Row Anomaly Flagging Rules Lookup Table, KNMI Technical Document, TN-OMIE-KNMI-950, KMNI, De Bilt, the Netherlands, 2010

Breider, T. J., Chipperfield, M. P., Richards, N. A. D., Carslaw, K. S., Mann, G. W., and Spracklen, D. V.: Impact of BrO on dimethylsulfide in the remote marine boundary layer, Geophys. Res. Lett., 37, 102807, https://doi.org/10.1029/2009GL040868, 2010.

Butchart, N.: The Brewer-Dobson circulation, Rev. Geophys., 52, 157-184, https://doi.org/10.1002/2013RG000448, 2014.

Carlton, A. G., Wiedinmyer, C., and Kroll, J. H.: A review of Secondary Organic Aerosol (SOA) formation from isoprene, Atmos.
Chem. Phys., 9, 4987-5005, https://doi.org/10.5194/acp-9-49872009, 2009.

Carver, G., Brown, P., and Wild, O.: The ASAD atmospheric chemistry integration package and chemical reaction database, Comput. Phys. Commun., 105, 197-215, 1997.

Chameides, W. L., Lindsay, R. W., Richardson, J., and Kiangs, C. S.: The role of biogenic hydrocarbons in urban photochemical smog: Atlanta as a case study, Science, 241, 1473-1475, https://doi.org/10.5194/acp-9-4987-2009, 1988.

Chipperfield, M. P.: New version of the TOMCAT/SLIMCAT offline chemical transport model: Intercomparison of stratospheric tracer experiments, Q. J. Roy. Meteor. Soc., 132, 1179-1203, https://doi.org/10.1256/qj.05.51, 2006.

Chipperfield, M. P., Dhomse, S. S., Feng, W., McKenzie, R. L., Velders, G., and Pyle, J. A.: Quantifying the ozone and ultraviolet benefits already achieved by the Montreal Protocol, Nat. Commun., 6, 7233, https://doi.org/10.1038/ncomms8233, 2015.

Dee, D. P., Uppala, S. M., Simmons, A. J., Berrisford, P., Poli, P., Kobayashi, S., Andrae, U., Balmaseda, M. A., Balsamo, G., Bauer, P., Bechtold, P., Beljaars, A. C. M., van de Berg, L., Bidlot, J., Bormann, N., Delsol, C., Dragani, R., Fuentes, M., Geer, A. J., Haimberger, L., Healy, S. B., Hersbach, H., Hólm, E. V., Isaksen, L., Kållberg, P., Köhler, M., Matricardi, M., McNally, A. P., Monge-Sanz, B. M., Morcrette, J.-J., Park, B.-K., Peubey, C., de Rosnay, P., Tavolato, C., Thépaut, J.-N., and Vitart, F.: The ERA-Interim reanalysis: configuration and performance of the data assimilation system, Q. J. Roy. Meteor. Soc., 137, 553-597, https://doi.org/10.1002/qj.828, 2011.

Deeter, M. N.: MOPITT (Measurements of Pollution in the Troposphere) Version 6 Product User's Guide, National Center for Atmospheric Research, Boulder, 2013.

Deeter, M. N., Edwards, D. P., Gille, J. C., Emmons, L. K., Francis, G., Ho, S.-P., Mao, D., Masters, D., Worden, H., Drummond, J. R., and Novelli, P. C.: The MOPITT version 4 CO product: Algorithm enhancements, validation, and long-term stability, J. Geophys. Res., 115, D07306, https://doi.org/10.1029/2009JD013005, 2010.

Deeter, M. N., Worden, H. M., Gille, J. C., Edwards, D. P., Mao, D., and Drummond, J. R.: MOPITT multispectral CO retrievals: Origins and effects of geophysical radiance errors, J. Geophys. Res., 116, D15303, https://doi.org/10.1029/2011JD015703, 2011.

Dhomse, S. S., Chipperfield, M. P., Feng, W., Ball, W. T., Unruh, Y. C., Haigh, J. D., Krivova, N. A., Solanki, S. K., and Smith, A. K.: Stratospheric $\mathrm{O}_{3}$ changes during 2001-2010: the small role of solar flux variations in a chemical transport model, Atmos. Chem. Phys., 13, 10113-10123, https://doi.org/10.5194/acp-1310113-2013, 2013.

Duncan, B. N., Logan, J. A., Bey, I., Megretskaia, I. A., Yantosca, R. M., Novelli, P. C., Jones, N. B., and Rinsland, C. P.: Global budget of CO, 1988-1997: Source estimates and validation with a global model, J. Geophys. Res., 112, D22301, https://doi.org/10.1029/2007JD008459, 2007.

Ellingsen, K., Gauss, M., Van Dingenen, R., Dentener, F. J., Emberson, L., Fiore, A. M., Schultz, M. G., Stevenson, D. S., Ashmore, M. R., Atherton, C. S., Bergmann, D. J., Bey, I., Butler, T., Drevet, J., Eskes, H., Hauglustaine, D. A., Isaksen, I. S. A., Horowitz, L. W., Krol, M., Lamarque, J. F., Lawrence, M. G., van Noije, T., Pyle, J., Rast, S., Rodriguez, J., Savage, N., Strahan, S., Sudo, K., Szopa, S., and Wild, O.: Global ozone 
and air quality: a multi-model assessment of risks to human health and crops, Atmos. Chem. Phys. Discuss., 8, 2163-2223, https://doi.org/10.5194/acpd-8-2163-2008, 2008.

Emmons, L. K., Arnold, S. R., Monks, S. A., Huijnen, V., Tilmes, S., Law, K. S., Thomas, J. L., Raut, J.-C., Bouarar, I., Turquety, S., Long, Y., Duncan, B., Steenrod, S., Strode, S., Flemming, J., Mao, J., Langner, J., Thompson, A. M., Tarasick, D., Apel, E. C., Blake, D. R., Cohen, R. C., Dibb, J., Diskin, G. S., Fried, A., Hall, S. R., Huey, L. G., Weinheimer, A. J., Wisthaler, A., Mikoviny, T., Nowak, J., Peischl, J., Roberts, J. M., Ryerson, T., Warneke, C., and Helmig, D.: The POLARCAT Model Intercomparison Project (POLMIP): overview and evaluation with observations, Atmos. Chem. Phys., 15, 6721-6744, https://doi.org/10.5194/acp-15-6721-2015, 2015.

Eskes, H. J. and Boersma, K. F.: Averaging kernels for DOAS totalcolumn satellite retrievals, Atmos. Chem. Phys., 3, 1285-1291, https://doi.org/10.5194/acp-3-1285-2003, 2003.

Evans, M. J. and Jacob, D. J.: Impact of new laboratory studies of $\mathrm{N}_{2} \mathrm{O}_{5}$ hydrolysis on global model budgets of tropospheric nitrogen oxides, ozone, and $\mathrm{OH}$, Geophys. Res. Lett., 32, 109813, https://doi.org/10.1029/2005GL022469, 2005.

Feng, W., Chipperfield, M. P., Dhomse, S., Monge-Sanz, B. M., Yang, X., Zhang, K., and Ramonet, M.: Evaluation of cloud convection and tracer transport in a three-dimensional chemical transport model, Atmos. Chem. Phys., 11, 5783-5803, https://doi.org/10.5194/acp-11-5783-2011, 2011.

Fiore, A. M., Dentener, F. J., Wild, O., Cuvelier, C., Schultz, M. G., Hess, P., Textor, C., Schulz, M., Doherty, R. M., Horowitz, L. W., MacKenzie, I. A., Sanderson, M. G., Shindell, D. T., Stevenson, D. S., Szopa, S., Van Dingenen, R., Zeng, G., Atherton, C., Bergmann, D., Bey, I., Carmichael, G., Collins, W. J., Duncan, B. N., Faluvegi, G., Folberth, G., Gauss, M., Gong, S., Hauglustaine, D., Holloway, T., Isaksen, I. S. A., Jacob, D. J., Jonson, J. E., Kaminski, J. W., Keating, T. J., Lupu, A., Marmer, E., Montanaro, V., Park, R. J., Pitari, G., Pringle, K. J., Pyle, J. A., Schroeder, S., Vivanco, M. G., Wind, P., Wojcik, G., Wu, S., and Zuber, A.: Multimodel estimates of intercontinental sourcereceptor relationships for ozone pollution, J. Geophys. Res., 114, D04301, https://doi.org/10.1029/2008JD010816, 2009.

Folberth, G. A., Hauglustaine, D. A., Lathière, J., and Brocheton, F.: Interactive chemistry in the Laboratoire de Météorologie Dynamique general circulation model: model description and impact analysis of biogenic hydrocarbons on tropospheric chemistry, Atmos. Chem. Phys., 6, 2273-2319, https://doi.org/10.5194/acp-6-2273-2006, 2006.

Fortems-Cheiney, A., Chevallier, F., Pison, I., Bousquet, P., Szopa, S., Deeter, M. N., and Clerbaux, C.: Ten years of $\mathrm{CO}$ emissions as seen from Measurements of Pollution in the Troposphere (MOPITT), J. Geophys. Res., 116, D05304, https://doi.org/10.1029/2010JD014416, 2011.

Franco, B., Mahieu, E., Emmons, L., Tzompa-Sosa, Z., Fischer, E., Sudo, K., Bovy, B., Conway, S., Griffin, D., Hannigan, J., and Strong, K.: Evaluating ethane and methane emissions associated with the development of oil and natural gas extraction in North America, Environ. Res. Lett., 11, 044010, https://doi.org/10.1088/1748-9326/11/4/044010, 2016.

Fuentes, J. D., Gu, L., Lerdau, M., Atkinson, R., Baldocchi, D., Bottenheim, J. W., Ciccioli, P., Lamb, B., Geron, C., Guenther, A., Sharkey, T. D., and Stockwell, W.: Biogenic hydro- carbons in the atmospheric boundary layer: a review, B. Am. Meteorol. Soc., 81, 1537-1575, https://doi.org/10.1175/15200477(2000)081<1537:BHITAB>2.3.CO;2, 2000.

Giannakopoulos, C., Chipperfield, M. P., Law, K. S., and Pyle, J. A.: Validation and intercomparison of wet and dry deposition schemes using $210 \mathrm{~Pb}$ in a global three-dimensional off-line chemical transport model, J. Geophys. Res., 104, 23761-23784, https://doi.org/10.1029/1999JD900392, 1999.

Gligorovski, S., Strekowski, R., Barbati, S., and Vione, D.: Environmental implications of hydroxyl radicals $\left({ }^{\bullet} \mathrm{OH}\right)$, Chem. Rev., 115, 13051-13092, https://doi.org/10.1021/cr500310b, 2015.

Guenther, A., Karl, T., Harley, P., Wiedinmyer, C., Palmer, P. I., and Geron, C.: Estimates of global terrestrial isoprene emissions using MEGAN (Model of Emissions of Gases and Aerosols from Nature), Atmos. Chem. Phys., 6, 3181-3210, https://doi.org/10.5194/acp-6-3181-2006, 2006.

Heard, D. E. and Pilling, M. J.: Measurement of $\mathrm{OH}$ and $\mathrm{HO}_{2}$ in the Troposphere, Chem. Rev., 103, 5163-5198, https://doi.org/10.1021/cr020522s, 2003.

Hollaway, M. J., Arnold, S. R., Challinor, A. J., and Emberson, L. D.: Intercontinental trans-boundary contributions to ozone-induced crop yield losses in the Northern Hemisphere, Biogeosciences, 9, 271-292, https://doi.org/10.5194/bg-9-2712012, 2012.

Holtslag, A. and Bolville, B.: Local versus nonlocal boundary layer diffusion in a global climate model, J. Climate, 6, 1825-1842, https://doi.org/10.1175/15200442(1993)006<1825:LVNBLD>2.0.CO;2, 1993.

Huijnen, V., Eskes, H. J., Poupkou, A., Elbern, H., Boersma, K. F., Foret, G., Sofiev, M., Valdebenito, A., Flemming, J., Stein, O., Gross, A., Robertson, L., D'Isidoro, M., Kioutsioukis, I., Friese, E., Amstrup, B., Bergstrom, R., Strunk, A., Vira, J., Zyryanov, D., Maurizi, A., Melas, D., Peuch, V.-H., and Zerefos, C.: Comparison of $\mathrm{OMI} \mathrm{NO}_{2}$ tropospheric columns with an ensemble of global and European regional air quality models, Atmos. Chem. Phys., 10, 3273-3296, https://doi.org/10.5194/acp10-3273-2010, 2010.

Irie, H., Boersma, K. F., Kanaya, Y., Takashima, H., Pan, X., and Wang, Z. F.: Quantitative bias estimates for tropospheric $\mathrm{NO}_{2}$ columns retrieved from SCIAMACHY, OMI, and GOME-2 using a common standard for East Asia, Atmos. Meas. Tech., 5, 2403-2411, https://doi.org/10.5194/amt-5-2403-2012, 2012.

Jacob, D. J.: Heterogeneous chemistry and tropospheric ozone, Atmos. Environ., 34, 2131-2159, https://doi.org/10.1016/S13522310(99)00462-8, 2000.

Jacob, D. J., Crawford, J. H., Maring, H., Clarke, A. D., Dibb, J. E., Emmons, L. K., Ferrare, R. A., Hostetler, C. A., Russell, P. B., Singh, H. B., Thompson, A. M., Shaw, G. E., McCauley, E., Pederson, J. R., and Fisher, J. A.: The Arctic Research of the Composition of the Troposphere from Aircraft and Satellites (ARCTAS) mission: design, execution, and first results, Atmos. Chem. Phys., 10, 5191-5212, https://doi.org/10.5194/acp10-5191-2010, 2010.

Kinnison, D. E., Brasseur, G. P., Walters, S., Garcia, R. R., Marsh, D. R., Sassi, F., Harvey, V. L., Randall, C. E., Emmons, L., Lamarque, J. F., Hess, P., Orlando, J. J., Tie, X. X., Randel, W., Pan, L. L., Gettelman, A., Granier, C., Diehl, T., Niemeier, U., and Simmons, A. J.: Sensitivity of chemical tracers to meteorological parameters in the MOZART- 
3 chemical transport model, J. Geophys. Res., 112, D20302, https://doi.org/10.1029/2006JD007879, 2007.

Kopacz, M., Jacob, D. J., Fisher, J. A., Logan, J. A., Zhang, L., Megretskaia, I. A., Yantosca, R. M., Singh, K., Henze, D. K., Burrows, J. P., Buchwitz, M., Khlystova, I., McMillan, W. W., Gille, J. C., Edwards, D. P., Eldering, A., Thouret, V., and Nedelec, P.: Global estimates of $\mathrm{CO}$ sources with high resolution by adjoint inversion of multiple satellite datasets (MOPITT, AIRS, SCIAMACHY, TES), Atmos. Chem. Phys., 10, 855-876, https://doi.org/10.5194/acp-10-855-2010, 2010.

Krol, M. and Lelieveld, J.: Can the variability in tropospheric $\mathrm{OH}$ be deduced from measurements of 1,1,1-trichloroethane (methyl chloroform)?, J. Geophys. Res., 108, 4125, https://doi.org/10.1029/2002JD002423, 2003.

Krol, M., van Leeuwen, P. J., and Lelieveld, J.: Global OH trend inferred from methylchloroform measurements, J. Geophys. Res., 103, 10697-10711, https://doi.org/10.1029/98JD00459, 1998.

Krol, M. C., Lelieveld, J., Oram, D. E., Sturrock, G. A., Penkett, S. A., Brenninkmeijer, C. A. M., Gros, V., Williams, J., and Scheeren, H. A.: Continuing emissions of methyl chloroform from Europe, Nature, 421, 131-135, https://doi.org/10.1038/nature01311, 2003.

Lamarque, J.-F., Bond, T. C., Eyring, V., Granier, C., Heil, A., Klimont, Z., Lee, D., Liousse, C., Mieville, A., Owen, B., Schultz, M. G., Shindell, D., Smith, S. J., Stehfest, E., Van Aardenne, J., Cooper, O. R., Kainuma, M., Mahowald, N., McConnell, J. R., Naik, V., Riahi, K., and van Vuuren, D. P.: Historical (1850-2000) gridded anthropogenic and biomass burning emissions of reactive gases and aerosols: methodology and application, Atmos. Chem. Phys., 10, 7017-7039, https://doi.org/10.5194/acp-10-7017-2010, 2010.

Law, K. S. and Pyle, J. A.: Modeling trace gas budgets in the troposphere: 1. Ozone and odd nitrogen, J. Geophys. Res.-Atmos., 98, 18377-18400, https://doi.org/10.1029/93JD01479, 1993.

Law, K. S., Plantevin, P. H., Shallcross, D. E., Rogers, H. L., Pyle, J. A., Grouhel, C., Thouret, V., and Marenco, A.: Evaluation of modeled $\mathrm{O}_{3}$ using Measurement of Ozone by Airbus In-Service Aircraft (MOZAIC) data, J. Geophys. Res.-Atmos., 103, 2572125737, https://doi.org/10.1029/98JD01482, 1998.

Lawrence, M. G., Jöckel, P., and von Kuhlmann, R.: What does the global mean $\mathrm{OH}$ concentration tell us?, Atmos. Chem. Phys., 1, 37-49, https://doi.org/10.5194/acp-1-37-2001, 2001.

Lelieveld, J. and Dentener, F. J.: What controls tropospheric ozone?, J. Geophys. Res., 105, 3531-3551, https://doi.org/10.1029/1999JD901011, 2000.

Lelieveld, J., Butler, T. M., Crowley, J. N., Dillon, T. J., Fischer, H., Ganzeveld, L., Harder, H., Lawrence, M. G., Martinez, M., Taraborrelli, D., and Williams, J.: Atmospheric oxidation capacity sustained by a tropical forest, Nature, 452, 737-740, https://doi.org/10.1038/nature06870, 2008.

Lelieveld, J., Evans, J., Fnais, M., Giannadaki, D., and Pozzer, A.: The contribution of outdoor air pollution sources to premature mortality on a global scale, Nature, 525, 367-371, https://doi.org/10.1038/nature15371, 2015.

Logan, J., Prather, M., Wofsy, S., and McElroy, M.: Tropospheric chemistry: A global perspective, J. Geophys. Res., 86, 72107254, https://doi.org/10.1029/JC086iC08p07210, 1981.

Mann, G. W., Carslaw, K. S., Spracklen, D. V., Ridley, D. A., Manktelow, P. T., Chipperfield, M. P., Pickering, S. J., and
Johnson, C. E.: Description and evaluation of GLOMAP-mode: a modal global aerosol microphysics model for the UKCA composition-climate model, Geosci. Model Dev., 3, 519-551, https://doi.org/10.5194/gmd-3-519-2010, 2010.

Mannschreck, K., Gilge, S., Plass-Duelmer, C., Fricke, W., and Berresheim, H.: Assessment of the applicability of $\mathrm{NO}-\mathrm{NO}_{2}-$ $\mathrm{O}_{3}$ photostationary state to long-term measurements at the Hohenpeissenberg GAW Station, Germany, Atmos. Chem. Phys., 4, 1265-1277, https://doi.org/10.5194/acp-4-1265-2004, 2004.

MCM: Master Chemical Mechanism v3.1, available at: http://mcm. leeds.ac.uk/MCM/, last access: 1 February 2004.

Meinshausen, M., Smith, S. J., Calvin, K., Daniel, J. S., Kainuma, M. L. T., Lamarque, J.-F., Matsumoto, K., Montzka, S. A., Raper, S. C. B., Riahi, K., Thomson, A., Velders, G. J. M., and van Vuuren, D. P.: The RCP greenhouse gas concentrations and their extensions from 1765 to 2300, Clim. Chang., 109, 213-241, https://doi.org/10.1007/s10584-011-0156-z, 2011.

Miles, G., Siddans, R., Kerridge, B., and Latter, B.: RAL Ozone Profile Algorithm Product User Guide Version 1.1, $2015 \mathrm{a}$.

Miles, G. M., Siddans, R., Kerridge, B. J., Latter, B. G., and Richards, N. A. D.: Tropospheric ozone and ozone profiles retrieved from GOME-2 and their validation, Atmos. Meas. Tech., 8, 385-398, https://doi.org/10.5194/amt-8-385-2015, 2015 b.

Mogili, P. K., Kleiber, P. D., Young, M. A., and Grassian, V. H.: $\mathrm{N}_{2} \mathrm{O}_{5}$ hydrolysis on the components of mineral dust and sea salt aerosol: Comparison study in an environmental aerosol reaction chamber, Atmos. Environ., 40, 7401-7408, https://doi.org/10.1016/j.atmosenv.2006.06.048, 2006.

Monks, S. A., Arnold, S. R., and Chipperfield, M. P.: Evidence for El Niño-Southern Oscillation (ENSO) influence on Arctic $\mathrm{CO}$ interannual variability through biomass burning emissions, Geophys. Res. Lett., 39, L14804, https://doi.org/10.1029/2012GL052512, 2012.

Monks, P. S., Archibald, A. T., Colette, A., Cooper, O., Coyle, M., Derwent, R., Fowler, D., Granier, C., Law, K. S., Mills, G. E., Stevenson, D. S., Tarasova, O., Thouret, V., von Schneidemesser, E., Sommariva, R., Wild, O., and Williams, M. L.: Tropospheric ozone and its precursors from the urban to the global scale from air quality to short-lived climate forcer, Atmos. Chem. Phys., 15, 8889-8973, https://doi.org/10.5194/acp-15-8889-2015, $2015 \mathrm{a}$.

Monks, S. A., Arnold, S. R., Emmons, L. K., Law, K. S., Turquety, S., Duncan, B. N., Flemming, J., Huijnen, V., Tilmes, S., Langner, J., Mao, J., Long, Y., Thomas, J. L., Steenrod, S. D., Raut, J. C., Wilson, C., Chipperfield, M. P., Diskin, G. S., Weinheimer, A., Schlager, H., and Ancellet, G.: Multi-model study of chemical and physical controls on transport of anthropogenic and biomass burning pollution to the Arctic, Atmos. Chem. Phys., 15, 3575-3603, https://doi.org/10.5194/acp-15-3575-2015, 2015 b.

Montzka, S. A., Krol, M., Dlugokencky, E., Hall, B., Jöckel, P., and Lelieveld, J.: Small interannual variability of global atmospheric hydroxyl, Science, 331, 67-69, https://doi.org/10.1126/science.1197640, 2011.

Naik, V., Voulgarakis, A., Fiore, A. M., Horowitz, L. W., Lamarque, J.-F., Lin, M., Prather, M. J., Young, P. J., Bergmann, D., Cameron-Smith, P. J., Cionni, I., Collins, W. J., Dalsøren, S. B., Doherty, R., Eyring, V., Faluvegi, G., Folberth, G. A., Josse, B., Lee, Y. H., MacKenzie, I. A., Nagashima, T., van Noije, T. P. C., Plummer, D. A., Righi, M., Rumbold, S. T., Skeie, R., Shindell, D. T., Stevenson, D. S., Strode, S., Sudo, K., Szopa, S., and Zeng, 
G.: Preindustrial to present-day changes in tropospheric hydroxyl radical and methane lifetime from the Atmospheric Chemistry and Climate Model Intercomparison Project (ACCMIP), Atmos. Chem. Phys., 13, 5277-5298, https://doi.org/10.5194/acp13-5277-2013, 2013

Novelli, P., Masarie, K., and Lang, P.: Distributions and recent changes of carbon monoxide in the lower troposphere, J. Geophys. Res., 103, 19015-19033, 1998.

Oleson, K. W., Lawrence, D. M., Bonan, G. B., Flanner, M. G., Kluzek, E., Lawrence, P. J., Levis, S., Swenson, S. C., Thornton, P. E., Dai, A., Decker, M., Dickinson, R., Feddema, J., Heald, C. L., Hoffman, F., Lamarque, J.-F., Mahowald, N., Niu, G.Y., Qian, T., Randerson, J., Running, S., Sakaguchi, K., Slater, A., Stockli, R., Wang, A., Yang, Z.-L., Zeng, X., and Zeng, X.: Technical Description of version 4.0 of the Community Land Model(CLM), Ncar technical note ncar/tn-478+str, NationalCenter for Atmospheric Research, Boulder, CO, 2010.

Oltmans, S. J. and Levy, H.: Surface ozone measurements from a global network, Atmos. Environ., 28, 9-24, https://doi.org/10.1016/1352-2310(94)90019-1, 1994.

Pandey Deolal, S., Henne, S., Ries, L., Gilge, S., Weers, U., Steinbacher, M., Staehelin, J., and Peter, T.: Analysis of elevated springtime levels of Peroxyacetyl nitrate (PAN) at the high Alpine research sites Jungfraujoch and Zugspitze, Atmos. Chem. Phys., 14, 12553-12571, https://doi.org/10.5194/acp-14-125532014, 2014.

Patra, P. K., Houweling, S., Krol, M., Bousquet, P., Belikov, D., Bergmann, D., Bian, H., Cameron-Smith, P., Chipperfield, M. P., Corbin, K., Fortems-Cheiney, A., Fraser, A., Gloor, E., Hess, P., Ito, A., Kawa, S. R., Law, R. M., Loh, Z., Maksyutov, S., Meng, L., Palmer, P. I., Prinn, R. G., Rigby, M., Saito, R., and Wilson, C.: TransCom model simulations of $\mathrm{CH}_{4}$ and related species: linking transport, surface flux and chemical loss with $\mathrm{CH}_{4}$ variability in the troposphere and lower stratosphere, Atmos. Chem. Phys., 11, 12813-12837, https://doi.org/10.5194/acp-11-128132011, 2011.

Patra, P. K., Krol, M. C., Montzka, S. A., Arnold, T., Atlas, E. L., Lintner, B. R., Stephens, B. B., Xiang, B., Elkins, J. W., Fraser, P. J., Ghosh, A., Hintsa, E. J., Hurst, D. F., Ishijima, K., Krummel, P. B., Miller, B. R., Miyazaki, K., Moore, F. L., Mühle, J., O’Doherty, S., Prinn, R. G., Steele, L. P., Takigawa, M., Wang, H. J., Weiss, R. F., Wofsy, S. C., and Young, D.: Observational evidence for interhemispheric hydroxyl-radical parity, Nature, 513, 219-223, https://doi.org/10.1038/nature13721, 2014.

Pétron, G., Granier, C., Khattatov, B., Yudin, V., Lamarque, J.-F., Emmons, L., Gille, J., and Edwards, D. P.: Monthly CO surface sources inventory based on the 2000-2001 MOPITT satellite data, Geophys. Res. Lett., 31, 121107 , https://doi.org/10.1029/2004GL020560, 2004.

Plass-Dülmer, C., Michl, K., Ruf, R., and Berresheim, H.: $\mathrm{C}_{2}-$ $\mathrm{C}_{8}$ Hydrocarbon measurement and quality control procedures at the Global Atmosphere Watch Observatory Hohenpeissenberg, J. Chromatogr. A, 953, 175-197, https://doi.org/10.1016/S00219673(02)00128-0, 2002.

Pope, R. J., Chipperfield, M. P., Savage, N. H., Ordóñez, C., Neal, L. S., Lee, L. A., Dhomse, S. S., Richards, N. A. D., and Keslake, T. D.: Evaluation of a regional air quality model using satellite column $\mathrm{NO}_{2}$ : treatment of observation errors and model boundary conditions and emissions, Atmos. Chem. Phys., 15, 5611-5626, https://doi.org/10.5194/acp-15-5611-2015, 2015.

Pope, R. J., Richards, N. A. D., Chipperfield, M. P., Moore, D. P., Monks, S. A., Arnold, S. R., Glatthor, N., Kiefer, M., Breider, T. J., Harrison, J. J., Remedios, J. J., Warneke, C., Roberts, J. M., Diskin, G. S., Huey, L. G., Wisthaler, A., Apel, E. C., Bernath, P. F., and Feng, W.: Intercomparison and evaluation of satellite peroxyacetyl nitrate observations in the upper tropospherelower stratosphere, Atmos. Chem. Phys., 16, 13541-13559, https://doi.org/10.5194/acp-16-13541-2016, 2016.

Pöschl, U., von Kuhlmann, R., Poisson, N., and Crutzen, P. J.: Development and intercomparison of condensed isoprene oxidation mechanisms for global atmospheric modeling, J. Atmos. Chem., 37, 29-52, 2000.

Prather, M.: Numerical advection by conservation of second-order moments, J. Geophys. Res., 91, 6671-6681, 1986.

Prinn, R. G., Huang, J., Weiss, R. F., Cunnold, D. M., Fraser, P. J., Simmonds, P. G., McCulloch, A., Harth, C., Salameh, P., O'Doherty, S., Wang, R. H. J., Porter, L., and Miller, B. R.: Evidence for substantial variations of atmospheric hydroxyl radicals in the past two decades, Science, 292, 1882-1888, https://doi.org/10.1126/science.1058673, 2001.

Ravishankara, A. R., Dunlea, E. J., Blitz, M. A., Dillon, T. J., Heard, D. E., Pilling, M. J., Strekowski, R. S., Nicovich, J. M., and Wine, P. H.: Redetermination of the rate coefficient for the reaction of $\mathrm{O}\left({ }^{1} \mathrm{D}\right)$ with $\mathrm{N}_{2}$, Geophys. Res. Lett., 29, 35-1-35-4, https://doi.org/10.1029/2001GL014850, 2002.

Read, K., Mahajan, A., Carpenter, L., Evans, M., Faria, B. Heard, D., Hopkins, J., Lee, J., Moller, S., Lewis, A., Mendes, L., McQuaid, J., Oetjen, H., Saiz-Lopez, A., Pilling, M., and Plane, J.: Extensive halogen-mediated ozone destruction over the tropical Atlantic Ocean, Nature, 453, 1232-1235, https://doi.org/10.1038/nature07035, 2008.

Richards, N. A. D., Arnold, S. R., Chipperfield, M. P., Miles, G., Rap, A., Siddans, R., Monks, S. A., and Hollaway, M. J.: The Mediterranean summertime ozone maximum: global emission sensitivities and radiative impacts, Atmos. Chem. Phys., 13, 2331-2345, https://doi.org/10.5194/acp-13-2331-2013, 2013.

Rodgers, C. D.: Retrieval of atmospheric temperature and composition from remote measurements of thermal radiation, Rev. Geophys., 14, 609-624, https://doi.org/10.1029/RG014i004p00609, 1976.

Sander, S. P., Golden, D. M., Kurylo, M. J., Moortgat, G. K., Wine, P. H., Ravishankara, A. R., Kolb, C. E., Molina, M. J., FinlaysonPitts, B. J., Huie, R. E., and Orkin, V. L.: Chemical Kinetics and Photochemical Data for Use in Atmospheric Studies Evaluation Number 15. JPL Publication 06-2, Tech. rep., Jet Propulsion Laboratory, Pasadena, USA, 2006.

Sander, S. P., Abbatt, J., Barker, J. R., Burkholder, J. B., Friedl, R. R., Golden, D. M., Huie, R. E., Kolb, C. E., Kurylo, M. J., Moortgat, G. K., Orkin, V. L., and Wine, P. H.: Chemical Kinetics and Photochemical Data for Use in Atmospheric Studies, Evaluation No. 17, JPL Publication 10-6, Jet Propulsion Laboratory, Pasadena, available at: http://jpldataeval.jpl.nasa.gov (last access: 9 August 2017), 2011.

Sanderson, M. G., Dentener, F. J., Fiore, A. M., Cuvelier, C., Keating, T. J., Zuber, A., Atherton, C. S., Bergmann, D. J., Diehl, T., Doherty, R. M., Duncan, B. N., Hess, P., Horowitz, L. W., Jacob, D. J., Jonson, J.-E., Kaminski, J. W., Lupu, 
A., MacKenzie, I. A., Mancini, E., Marmer, E., Park, R., Pitari, G., Prather, M. J., Pringle, K. J., Schroeder, S., Schultz, M. G., Shindell, D. T., Szopa, S., Wild, O., and Wind, P.: A multi-model study of the hemispheric transport and deposition of oxidised nitrogen, Geophys. Res. Lett., 35, 117815, https://doi.org/10.1029/2008GL035389, 2008.

Saunders, S. M., Jenkin, M. E., Derwent, R. G., and Pilling, M. J.: Protocol for the development of the Master Chemical Mechanism, MCM v3 (Part A): tropospheric degradation of nonaromatic volatile organic compounds, Atmos. Chem. Phys., 3, 161-180, https://doi.org/10.5194/acp-3-161-2003, 2003.

Shindell, D. T., Faluvegi, G., Stevenson, D. S., Krol, M. C., Emmons, L. K., Lamarque, J.-F., Pétron, G., Dentener, F. J., Ellingsen, K., Schultz, M. G., Wild, O., Amann, M., Atherton, C. S., Bergmann, D. J., Bey, I., Butler, T., Cofala, J., Collins, W. J., Derwent, R. G., Doherty, R. M., Drevet, J., Eskes, H. J., Fiore, A. M., Gauss, M., Hauglustaine, D. A., Horowitz, L. W., Isaksen, I. S. A., Lawrence, M. G., Montanaro, V., Müller, J.-F., Pitari, G., Prather, M. J., Pyle, J. A., Rast, S., Rodriguez, J. M., Sanderson, M. G., Savage, N. H., Strahan, S. E., Sudo, K., Szopa, S., Unger, N., van Noije, T. P. C., and Zeng, G.: Multimodel simulations of carbon monoxide: Comparison with observations and projected near-future changes, J. Geophys. Res., 111, D19306, https://doi.org/10.1029/2006JD007100, 2006.

Shindell, D. T., Chin, M., Dentener, F., Doherty, R. M., Faluvegi, G., Fiore, A. M., Hess, P., Koch, D. M., MacKenzie, I. A., Sanderson, M. G., Schultz, M. G., Schulz, M., Stevenson, D. S., Teich, H., Textor, C., Wild, O., Bergmann, D. J., Bey, I., Bian, H., Cuvelier, C., Duncan, B. N., Folberth, G., Horowitz, L. W., Jonson, J., Kaminski, J. W., Marmer, E., Park, R., Pringle, K. J., Schroeder, S., Szopa, S., Takemura, T., Zeng, G., Keating, T. J., and Zuber, A.: A multi-model assessment of pollution transport to the Arctic, Atmos. Chem. Phys., 8, 5353-5372, https://doi.org/10.5194/acp-8-5353-2008, 2008.

Sofen, E. D., Bowdalo, D., Evans, M. J., Apadula, F., Bonasoni, P., Cupeiro, M., Ellul, R., Galbally, I. E., Girgzdiene, R., Luppo, S., Mimouni, M., Nahas, A. C., Saliba, M., and Tørseth, K.: Gridded global surface ozone metrics for atmospheric chemistry model evaluation, Earth Syst. Sci. Data, 8, 4159, https://doi.org/10.5194/essd-8-41-2016, 2016.

Spivakovsky, C. M., Logan, J. A., Montzka, S. A., Balkanski, Y. J., Foreman-Fowler, M., Jones, D. B. A., Horowitz, L. W., Fusco, A. C., Brenninkmeijer, C. A. M., Prather, M. J., Wofsy, S. C., and McElroy, M. B.: Three-dimensional climatological distribution of tropospheric $\mathrm{OH}$ : Update and evaluation, J. Geophys. Res., 105, 8931-8980, 2000.

Squire, O. J., Archibald, A. T., Griffiths, P. T., Jenkin, M. E., Smith, D., and Pyle, J. A.: Influence of isoprene chemical mechanism on modelled changes in tropospheric ozone due to climate and land use over the 21st century, Atmos. Chem. Phys., 15, 5123-5143, https://doi.org/10.5194/acp-15-5123-2015, 2015.

Stevenson, D. S., Dentener, F. J., Schultz, M. G., Ellingsen, K., van Noije, T. P. C., Wild, O., Zeng, G., Amann, M., Atherton, C. S., Bell, N., Bergmann, D. J., Bey, I., Butler, T., Cofala, J., Collins, W. J., Derwent, R. G., Doherty, R. M., Drevet, J., Eskes, H. J., Fiore, A. M., Gauss, M., Hauglustaine, D. A., Horowitz, L. W., Isaksen, I. S. A., Krol, M. C., Lamarque, J.-F., Lawrence, M. G., Montanaro, V., Müller, J.-F., Pitari, G., Prather, M. J., Pyle, J. A., Rast, S., Rodriguez, J. M., Sanderson, M. G.,
Savage, N. H., Shindell, D. T., Strahan, S. E., Sudo, K., and Szopa, S.: Multimodel ensemble simulations of present-day and near-future tropospheric ozone, J. Geophys. Res., 111, D08301, https://doi.org/10.1029/2005JD006338, 2006.

Stocker, T., Qin, D., Plattner, G.-K., Tignor, M., Allen, S., Boschung, J., Nauels, A., Xia, Y., Bex, V., and Midgley, P., eds.: IPCC, 2013: Climate Change 2013: The Physical Science Basis. Contribution of Working Group I to the Fifth Assessment Report of the Intergovernmental Panel on Climate Change, Cambridge University Press, Cambridge, UK and New York, NY, USA, 2013.

Stockwell, D., Giannakopoulos, C., Plantevin, P.-H., Carver, G., Chipperfield, M., Law, K., Pyle, J., Shallcross, D., and Wang, K.-Y.: Modelling $\mathrm{NO}_{x}$ from lightning and its impact on global chemical fields, Atmos. Environ., 33, 4477-4493, https://doi.org/10.1016/S1352-2310(99)00190-9, 1999.

Strode, S. A., Duncan, B. N., Yegorova, E. A., Kouatchou, J., Ziemke, J. R., and Douglass, A. R.: Implications of carbon monoxide bias for methane lifetime and atmospheric composition in chemistry climate models, Atmos. Chem. Phys., 15, 11789-11805, https://doi.org/10.5194/acp-15-11789-2015, 2015.

Sukhodolov, T., Rozanov, E., Ball, W. T., Bais, A., Tourpali, K., Shapiro, A. I., Telford, P., Smyshlyaev, S., Fomin, B., Sander R., Bossay, S., Bekki, S., Marchand, M., Chipperfield, M. P., Dhomse, S., Haigh, J. D., Peter, T., and Schmutz, W.: Evaluation of simulated photolysis rates and their response to solar irradiance variability, J. Geophys. Res., 121, 6066-6084, https://doi.org/10.1002/2015JD024277, 2015JD024277, 2016.

Tiedtke, M.: A comprehensive mass flux scheme for cumulus parameterization in large-scale models, Mon. Weather Rev., 117, 1779-1800, 1989.

Tilmes, S., Lamarque, J.-F., Emmons, L. K., Conley, A., Schultz, M. G., Saunois, M., Thouret, V., Thompson, A. M., Oltmans, S. J., Johnson, B., and Tarasick, D.: Technical Note: Ozonesonde climatology between 1995 and 2011: description, evaluation and applications, Atmos. Chem. Phys., 12, 74757497, https://doi.org/10.5194/acp-12-7475-2012, 2012.

Tilmes, S., Lamarque, J.-F., Emmons, L. K., Kinnison, D. E., Ma, P.-L., Liu, X., Ghan, S., Bardeen, C., Arnold, S., Deeter, M., Vitt, F., Ryerson, T., Elkins, J. W., Moore, F., Spackman, J. R., and Val Martin, M.: Description and evaluation of tropospheric chemistry and aerosols in the Community Earth System Model (CESM1.2), Geosci. Model Dev., 8, 1395-1426, https://doi.org/10.5194/gmd8-1395-2015, 2015.

Tilmes, S., Lamarque, J.-F., Emmons, L. K., Kinnison, D. E., Marsh, D., Garcia, R. R., Smith, A. K., Neely, R. R., Conley, A., Vitt, F., Val Martin, M., Tanimoto, H., Simpson, I., Blake, D. R., and Blake, N.: Representation of the Community Earth System Model (CESM1) CAM4-chem within the ChemistryClimate Model Initiative (CCMI), Geosci. Model Dev., 9, 18531890, https://doi.org/10.5194/gmd-9-1853-2016, 2016.

Tyndall, G. S., Cox, R. A., Granier, C., Lesclaux, R., Moortgat, G. K., Pilling, M. J., Ravishankara, A. R., and Wallington, T. J.: Atmospheric chemistry of small organic peroxy radicals, J. Geophys. Res., 106, 12157-12182, https://doi.org/10.1029/2000JD900746, 2001.

ValMartin, M., Heald, C. L., and Arnold, S. R.: Coupling dry deposition to vegetation phenology in the Com- 
munity Earth System Model: Implications for the simulation of surface $\mathrm{O}_{3}$, Geophys. Res. Lett., 41, 2988-2996, https://doi.org/10.1002/2014GL059651, 2014.

van der Werf, G. R., Randerson, J. T., Giglio, L., Collatz, G. J., Mu, M., Kasibhatla, P. S., Morton, D. C., DeFries, R. S., Jin, Y., and van Leeuwen, T. T.: Global fire emissions and the contribution of deforestation, savanna, forest, agricultural, and peat fires (1997-2009), Atmos. Chem. Phys., 10, 11707-11735, https://doi.org/10.5194/acp-10-11707-2010, 2010.

von Kuhlmann, R.: Photochemistry of Tropospheric Ozone, its Precursors and the Hydroxyl Radical: A 3D-Modeling Study Considering Non-Methane Hydrocarbons, Ph.D. thesis, Johannes Gutenberg-Universitat Mainz, Mainz, Germany, 2001.

Voulgarakis, A., Naik, V., Lamarque, J.-F., Shindell, D. T., Young, P. J., Prather, M. J., Wild, O., Field, R. D., Bergmann, D., CameronSmith, P., Cionni, I., Collins, W. J., Dalsøren, S. B., Doherty, R. M., Eyring, V., Faluvegi, G., Folberth, G. A., Horowitz, L. W., Josse, B., MacKenzie, I. A., Nagashima, T., Plummer, D. A., Righi, M., Rumbold, S. T., Stevenson, D. S., Strode, S. A., Sudo, K., Szopa, S., and Zeng, G.: Analysis of present day and future $\mathrm{OH}$ and methane lifetime in the ACCMIP simulations, Atmos. Chem. Phys., 13, 2563-2587, https://doi.org/10.5194/acp13-2563-2013, 2013.

Wang, J. S., McElroy, M. B., Logan, J. A., Palmer, P. I., Chameides, W. L., Wang, Y., and Megretskaia, I. A.: A quantitative assessment of uncertainties affecting estimates of global mean $\mathrm{OH}$ derived from methyl chloroform observations, J. Geophys. Res., 113, D12302, https://doi.org/10.1029/2007JD008496, 2008.

Wang, K.-Y. and Shallcross, D.: Modelling terrestrial biogenic isoprene fluxes and their potential impact on global chemical species using a coupled LSM-CTM model, Atmos. Environ., 34, 2909-2925, https://doi.org/10.1016/S1352-2310(99)005257, 2000.

Watanabe, F., Uchino, O., Joo, Y., Aono, M., Higashijima, K., Hirano, Y., Tsuboi, K., and Suda, K.: Interannual variation of growth rate of atmospheric carbon dioxide concentration observed at the JMA's three monitoring stations: Large increase in concentration of atmospheric carbon dioxide in 1998, J. Meteorol. Soc. Jpn., 78, 673-682, 2000.

Wiedinmyer, C., Akagi, S. K., Yokelson, R. J., Emmons, L. K., AlSaadi, J. A., Orlando, J. J., and Soja, A. J.: The Fire INventory from NCAR (FINN): a high resolution global model to estimate the emissions from open burning, Geosci. Model Dev., 4, 625641, https://doi.org/10.5194/gmd-4-625-2011, 2011.

Wilson, C., Chipperfield, M. P., Gloor, M., and Chevallier, F.: Development of a variational flux inversion system (INVICAT v1.0) using the TOMCAT chemical transport model, Geosci. Model Dev., 7, 2485-2500, https://doi.org/10.5194/gmd-7-2485-2014, 2014.
Young, P.: The influence of biogenic isoprene emissions on atmospheric chemistry: A model study for present and future atmospheres, Ph.D. thesis, University of Cambridge, Cambridge, 2007.

Young, P. J., Archibald, A. T., Bowman, K. W., Lamarque, J.-F., Naik, V., Stevenson, D. S., Tilmes, S., Voulgarakis, A., Wild, O., Bergmann, D., Cameron-Smith, P., Cionni, I., Collins, W. J., Dalsøren, S. B., Doherty, R. M., Eyring, V., Faluvegi, G., Horowitz, L. W., Josse, B., Lee, Y. H., MacKenzie, I. A., Nagashima, T., Plummer, D. A., Righi, M., Rumbold, S. T., Skeie, R. B., Shindell, D. T., Strode, S. A., Sudo, K., Szopa, S., and Zeng, G.: Preindustrial to end 21 st century projections of tropospheric ozone from the Atmospheric Chemistry and Climate Model Intercomparison Project (ACCMIP), Atmos. Chem. Phys., 13, 2063 2090, https://doi.org/10.5194/acp-13-2063-2013, 2013.

Yu, S., Mathur, R., Sarwar, G., Kang, D., Tong, D., Pouliot, G., and Pleim, J.: Eta-CMAQ air quality forecasts for $\mathrm{O}_{3}$ and related species using three different photochemical mechanisms (CB4, CB05, SAPRC-99): comparisons with measurements during the 2004 ICARTT study, Atmos. Chem. Phys., 10, 30013025, https://doi.org/10.5194/acp-10-3001-2010, 2010.

Zellweger, C., Forrer, J., Hofer, P., Nyeki, S., Schwarzenbach, B., Weingartner, E., Ammann, M., and Baltensperger, U.: Partitioning of reactive nitrogen $\left(\mathrm{NO}_{y}\right)$ and dependence on meteorological conditions in the lower free troposphere, Atmos Chem. Phys., 3, 779-796, https://doi.org/10.5194/acp-3-7792003, 2003.

Zhang, K., Wan, H., Zhang, M., and Wang, B.: Evaluation of the atmospheric transport in a GCM using radon measurements: sensitivity to cumulus convection parameterization, Atmos. Chem Phys., 8, 2811-2832, https://doi.org/10.5194/acp-8-2811-2008, 2008.

Zhang, Q., Streets, D. G., Carmichael, G. R., He, K. B., Huo, H., Kannari, A., Klimont, Z., Park, I. S., Reddy, S., Fu, J. S., Chen, D., Duan, L., Lei, Y., Wang, L. T., and Yao, Z. L.: Asian emissions in 2006 for the NASA INTEX-B mission, Atmos. Chem. Phys., 9, 5131-5153, https://doi.org/10.5194/acp-9-5131-2009, 2009.

Ziemke, J. R., Chandra, S., Labow, G. J., Bhartia, P. K., Froidevaux, L., and Witte, J. C.: A global climatology of tropospheric and stratospheric ozone derived from Aura OMI and MLS measurements, Atmos. Chem. Phys., 11, 9237-9251, https://doi.org/10.5194/acp-11-9237-2011, 2011. 